\title{
Arrovian Social Choice Theory on Economic Domains
}

\author{
by
}

Michel Le Breton and John A. Weymark

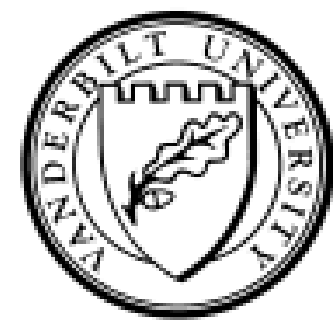

Working Paper No. 02-W06R

April 2002

Revised September 2003

\section{DEPARTMENT OF ECONOMICS \\ VANDERBILT UNIVERSITY \\ NASHVILLE, TN 37235}

www.vanderbilt.edu/econ 


\title{
Arrovian Social Choice Theory on Economic Domains*
}

\author{
by \\ Michel Le Breton \\ GREMAQ and IDEI, \\ Université de Toulouse 1 , \\ Manufacture des Tabacs, Bat F, \\ 21, Allée de Brienne 31000, Toulouse, France \\ (e-mail: lebreton@cict.fr) \\ and \\ John A. Weymark \\ Department of Economics, Box 1819, Station B, \\ Vanderbilt University, Nashville, TN 37235, U.S.A. \\ (e-mail: john.weymark@vanderbilt.edu)
}

April 2002

Revised, September 2003

This survey has been prepared for inclusion in K. J. Arrow, A. K. Sen, and K. Suzumura, eds., Handbook of Social Choice and Welfare, Volume 2 (North-Holland: Amsterdam).

*This chapter incorporates some material that has previously appeared in Le Breton and Weymark (1996). We are grateful to Lars Ehlers, Marc Fleurbaey, and Thierry Marchant for their comments. Preliminary versions of this chapter served as the basis for a set of lectures given at EUREQua, Université Paris 1 (Panthéon-Sorbonne) and at the Workshop on Mathematical Models of Individual and Public Choice held at the Institute for Mathematical Behavioral Sciences of the University of California at Irvine. 


\begin{abstract}
"Arrovian Social Choice Theory on Economic Domains"

by

Michel Le Breton and John A. Weymark
\end{abstract}

This article surveys the literature that investigates the consistency of Arrow's social choice axioms when his unrestricted domain assumptions are replaced by domain conditions that incorporate the restrictions on agendas and preferences encountered in economic environments. Both social welfare functions and social choice correspondences are considered.

Journal of Economic Literature classification number: D71.

Keywords and phrases: social choice, Arrow's Theorem, restricted domains. 


\section{Introduction}

A social welfare function specifies a social ordering of a set of alternatives for each profile of individual preferences in some domain of preference profiles. Arrow's Theorem [see Arrow (1963)] demonstrates that it is impossible for a social welfare function to satisfy Independence of Irrelevant Alternatives (the social ranking of a pair of alternatives only depends on the individual rankings of these alternatives), Weak Pareto (if everyone strictly prefers one alternative to a second, then so does society), and Nondictatorship (nobody's strict preferences are always respected) if the preference domain is unrestricted and there are at least three alternatives being ranked.

This theorem is not directly applicable to economic problems. In economic models, both the social alternatives and the individual preferences exhibit considerable structure. For example, (i) alternatives could be allocations of private goods with individuals restricted to having selfish, continuous, monotonic, and convex preferences, (ii) alternatives could be vectors of public goods with individual preferences required to be continuous, monotonic, and convex, or (iii) alternatives could be lotteries with preferences required to satisfy the axioms of expected utility theory. In each of these examples, the domain of admissible preference profiles is restricted, in contrast to Arrow's Theorem in which it is assumed that the preference domain is unrestricted.

Arrow's Theorem can also be formulated in terms of a social choice correspondence. In this case, the objective is to choose a set of socially optimal alternatives from a feasible subset of the alternatives called an agenda. A social choice correspondence specifies a nonempty subset of the agenda for each admissible preference profile and each admissible agenda. In its choicetheoretic formulation, Arrow's Theorem shows that Arrow's Choice Axiom (for a fixed preference profile, if agenda $A$ is a subset of agenda $B$ and some alternative from $A$ is chosen when the agenda is $B$, then the set of alternatives chosen from $A$ consists of the restriction to $A$ of the set of alternatives chosen from $B$ ), Independence of Infeasible Alternatives (the social choice only depends on the preferences for feasible alternatives), and the choice correspondence versions of Weak Pareto (only weakly Pareto optimal alternatives are chosen) and Nondictatorship (the chosen alternatives are not always a subset of one individual's best feasible alternatives) are inconsistent if the

preference domain is unrestricted and the agenda domain includes all the 
two and three element subsets of the universal set. ${ }^{1}$

The requirement that some of the agendas are finite is unnatural in many economic problems. For example, an agenda could be an Edgeworth box in an exchange economy or, alternatively, all of the allocations that are feasible for a production economy with given production technologies and resource endowment. By varying the technologies and/or the endowment, new agendas are obtained.

In this chapter, we survey the literature that investigates the consistency of Arrow's axioms when natural economic restrictions are placed on the universal set of alternatives, on preferences, and on agendas. We consider social welfare functions in Part I and social choice correspondences in Part II.

Prior to Arrow (1951), social choice theory focused on the properties of particular social decision procedures, such as majority rule. One of the innovative features of Arrow's work is that it shifted attention from the analysis of a given social decision rule to the question of whether any social decision procedure can satisfy a prespecified list of appealing properties. The answer to this question depends on the domain for which the social choice rule is meant to apply. Domains for which the Arrow axioms can be jointly satisfied are called Arrow-consistent.

The literature on the Arrow-consistency of the preference domain of a social welfare function has two main branches. In one branch of this literature, the objective is to find necessary and sufficient conditions for the domain to be Arrow-consistent when little or no a priori structure is placed on the set of alternatives. Not much is known about the implications of this line of research for economic domains, so it will not be considered in this survey. ${ }^{2}$

In the other branch of this literature, the objective is more modest. Instead of attempting to completely characterize the preference domains that are Arrow-consistent, the objective is to determine whether the preference domain in a specific economic problem is Arrow-consistent and, if so, to identify social welfare functions that satisfy all of the Arrow axioms on this domain. This research programme has been aided by the development of various sufficient conditions for a preference domain to be Arrow-inconsistent

\footnotetext{
${ }^{1}$ For some agenda domains, Arrow's Choice Axiom is the choice-theoretic analogue of Arrow's assumption that social preferences are orderings.

${ }^{2}$ See Gaertner $(2001 ; 2002)$ for discussions of this literature. Some of of the contributions to this literature assume that every individual exhibits a strict preference between any pair of alternatives or assume that the set of alternatives is finite, neither of which is a natural assumption in economic problems when the alternatives include divisible goods.
} 
that can be applied in a wide range of economic applications.

While, in retrospect, the work of Black (1948) on single-peaked preferences when the set of alternatives is one-dimensional and of Arrow (1951, Chapter VI) and Blau (1957) on the allocation of multiple private goods can be seen as contributing to this research programme, it was the pathbreaking contribution of Kalai, Muller, and Satterthwaite (1979) that set the stage for most of the subsequent developments on the Arrow-consistency of domains of economic preferences. ${ }^{3}$ They introduced the concept of a saturating domain and showed that it is a sufficient condition for the preference domain of a social welfare function to be Arrow-inconsistent when individuals are required to have the same set of admissible preferences. The method of proof used to establish this theorem is now known as the local approach. Some version of this methodology is used to prove most of the results we survey in Part I. As we shall see, except when alternatives are one-dimensional and preferences are single-peaked, it is typically the case that some version of Arrow's impossibility theorem holds for economic domains of interest.

The research on the consistency of Arrow's axioms on economic domains when the social choice correspondence framework is used originates with Bailey (1979), who questioned the relevance of Arrow's Theorem for exchange economies. Here, the results are more mixed. While the choice-theoretic versions of Arrow's axioms are inconsistent on many economic domains, a number of domains have been identified for which they are consistent. In contrast to the research on social welfare functions, the strategies used to determine whether various economic domains for social choice correspondences are Arrow-consistent or not have largely been domain-specific. As a consequence, the literature surveyed in Part II is less well-developed than the literature surveyed in Part I.

We begin Part I with a review of Arrow's Theorem for social welfare functions. Single-peaked preferences on a one-dimensional set of alternatives are considered in Section 3. In Section 4, we discuss saturating domains and the local approach. We illustrate the application of the local approach in Section 5 by showing that a number of economic domains for public alternatives are saturating. In Section 6, we discuss a topological approach to

\footnotetext{
${ }^{3}$ Also influential is the earlier unpublished work of Maskin (1976). Maskin showed that when there are at least two private goods, Weak Pareto and Nondictatorship are inconsistent with a nonnegative responsiveness condition (a strengthening of Independence of Irrelevant Alternatives) on the domain of selfish, continuous, monotonic, and convex preferences if each individual consumption set is a positive orthant.
} 
identifying Arrow-consistent domains due to Redekop (1991). Private goods are considered in Sections 7, 8, and 9. In Section 10, we discuss problems in which the set of alternatives does not have a Cartesian structure. This situation arises when feasibility constraints place restrictions on the set of alternatives, as is the case, for example, in exchange economies. In Section 11, we consider some implications of requiring a social welfare function to generate socially-best alternatives on some subsets of alternatives.

Part II begins by describing a choice-theoretic version of Arrow's Theorem. This is followed in Section 13 by a discussion of a number of problems in which the agenda domain is restricted, but the preference domain is not. This structure is exhibited by some voting models. In Section 14, we present a possibility theorem for a one-dimensional set of alternatives when preferences are single-peaked and agendas are compact intervals. In Section 15, we discuss some possibility theorems for multidimensional sets of alternatives when preferences are representable by analytic utility functions. Euclidean spatial preferences have this property. In Section 16, we consider some classical domains of spatial and economic preferences when agendas satisfy natural feasibility restrictions. In Section 17, the implications of a stronger independence condition are discussed for models similar to the ones considered in Section 16. Some concluding remarks are presented in Section 18.

Any survey of the literature on Arrovian social choice theory on economic domains would be incomplete without some discussion of the proof strategies that have been employed. Accordingly, for most of the results we discuss, we either present a proof or we provide heuristic arguments that convey the main intuition of a formal proof. By proceeding in this way, we hope that the reader will gain some appreciation for why significant progress has been in understanding when the domain of a social welfare function is Arrowinconsistent and why less progress has been made in understanding when Arrow's axioms for social choice correspondences are consistent.

\section{Part I: Social welfare functions}

\section{Arrow's theorem}

Almost all of the research that we survey builds on Arrow's [Arrow (1951; 1963)] classic work. In this section, we provide a brief introduction to Ar- 
row's impossibility theorem for social welfare functions. For more complete discussions, see, for example, Sen (1970) or Campbell and Kelly (2002).

We consider a finite set of individuals $N=\{1, \ldots, n\}$ with $n \geq 2$ and a universal set of alternatives $X . \mathcal{R}$ is the set of all orderings of $X$; i.e., $\mathcal{R}$ is the set of all reflexive, complete, and transitive binary relations on $X$. For each ordering $R$ in $\mathcal{R}$, strict preference $P$ and indifference $I$ are defined in the usual way: (a) $x P y$ if and only if $x R y$ and $\neg(y R x)$ and (b) $x I y$ if and only if $x R y$ and $y R x$. Each person has a preference ordering $R_{i} \in \mathcal{R}$ on $X$. A preference profile $\mathbf{R}=\left(R_{1}, \ldots, R_{n}\right)$ is an $n$-tuple of individual preference orderings on $X$. Two preferences $R^{1}, R^{2} \in \mathcal{R}$ coincide on $A \subseteq X$ if for all $x, y \in A, x R^{1} y \leftrightarrow x R^{2} y$. ${ }^{4}$ Two profiles $\mathbf{R}^{1}, \mathbf{R}^{2} \in \mathcal{R}^{n}$ coincide on $A \subseteq X$ if $R_{i}^{1}$ and $R_{i}^{2}$ coincide on $A$ for all $i \in N$.

The collection of admissible profiles $\mathcal{D}$, a nonempty subset of $\mathcal{R}^{n}$, is called the preference domain. Collective decisions are only required for profiles in the preference domain. A social welfare function on $\mathcal{D}$ is a mapping $F: \mathcal{D} \rightarrow \mathcal{R}$ that assigns a social preference ordering $F(\mathbf{R})$ of $X$ to each admissible profile $\mathbf{R}$. To simplify the notation, henceforth $F(\mathbf{R})$ is denoted as $R$.

Arrow (1951) supposed that the social welfare function must be determined before the individual preferences are known. If no structure is assumed about what form these preferences might take (other than that they are orderings), the social welfare function needs to be able to determine a social ordering of the alternatives for any conceivable preference profile.

Unrestricted Preference Domain. $\mathcal{D}=\mathcal{R}^{n}$.

In addition to his domain assumption, Arrow proposed three properties for a social welfare function to satisfy. The first of these axioms requires the social ranking of any pair of alternatives to depend only on the individual rankings of these alternatives.

Independence of Irrelevant Alternatives (IIA). For all $x, y \in X$ and all $\mathbf{R}^{1}, \mathbf{R}^{2} \in \mathcal{D}$, if $\mathbf{R}^{1}$ and $\mathbf{R}^{2}$ coincide on $\{x, y\}$, then $R^{1}$ and $R^{2}$ coincide on $\{x, y\}$.

Arrow's Weak Pareto axiom requires the social welfare function to respect unanimous strict rankings on pairs of alternatives.

\footnotetext{
${ }^{4}$ We distinguish between $\subseteq$ (weak set inclusion) and $\subset$ (strict set inclusion).
} 
Weak Pareto (WP). For all $x, y \in X$ and all $\mathbf{R} \in \mathcal{D}$, if $x P_{i} y$ for all $i \in N$, then $x P y$.

An individual $d \in N$ is a dictator on the ordered pair $(x, y) \in X^{2}$ if $x P y$ for all $\mathbf{R} \in \mathcal{D}$ such that $x P_{d} y$. An individual $d \in N$ is a dictator on the subset $A \subseteq X$ if $d$ is a dictator on the ordered pair $(x, y)$ for all $x, y \in A$. An individual $d \in N$ is a dictator if $d$ is a dictator on $X$. A social welfare function is dictatorial if there exists a dictator. Note that a dictatorial social welfare function only needs to respect the dictator's strict preferences. Arrow's final axiom requires the social welfare function to be nondictatorial.

Nondictatorship (ND). There is no dictator.

Detailed discussions of these axioms may be found in Sen (1970). As noted in Section 1, an Arrow-consistent domain for a social welfare function is a preference domain for which there exists a social welfare function satisfying IIA, WP, and ND. ${ }^{5}$ If no social welfare function exists for which these three axioms are satisfied, then the preference domain is Arrow-inconsistent.

If a preference domain is Arrow-consistent, one may want to see if it is possible for a social welfare function to satisfy further desirable properties. For example, Weak Pareto could be strengthened to Strong Pareto, the requirement that if nobody prefers $y$ to $x$ and somebody prefers $x$ to $y$, then $x$ should be socially preferred to $y$.

Strong Pareto (SP). For all $x, y \in X$ and all $\mathbf{R} \in \mathcal{D}$, if $x R_{i} y$ for all $i \in N$ and $x P_{j} y$ for some $j \in N$, then $x P y$.

Nondictatorship could be strengthened by requiring the social welfare function to treat individuals symmetrically in the sense that if we permute the individual preferences, then the social ordering is unchanged.

Anonymity (ANON). For all $\mathbf{R}^{1}, \mathbf{R}^{2} \in \mathcal{D}$, if $\mathbf{R}^{1}$ is a permutation of $\mathbf{R}^{2}$, then $F\left(\mathbf{R}^{1}\right)=F\left(\mathbf{R}^{2}\right)$.

Arrow's Theorem shows that it is impossible for a social welfare function to satisfy his three axioms when the preference domain is unrestricted. In other words, the domain $\mathcal{R}^{n}$ is Arrow-inconsistent.

\footnotetext{
${ }^{5}$ This terminology is due to Redekop (1991). This definition does not explicitly mention the set of alternatives $X$ being considered. However, $X$ can always be inferred from knowledge of the preference domain.
} 
Theorem 1. If $|X| \geq 3$, there is no social welfare function with an unrestricted preference domain that satisfies IIA, WP, and ND. ${ }^{6}$

Standard proofs of the social welfare function version of Arrow's Theorem, such as the one found in Sen (1970), show that a social welfare function must be dictatorial if it satisfies the other assumptions of Arrow's Theorem. Before describing the main steps in the proof of Arrow's Theorem, we need to introduce some further definitions. A nonempty group $G \subseteq N$ is almost decisive for the ordered pair $(x, y) \in X^{2}$ if $x$ is socially strictly preferred to $y$ when everyone in $G$ strictly prefers $x$ to $y$ and everyone else has the opposite strict ranking. $G$ is decisive for the ordered pair $(x, y) \in X^{2}$ if $x$ is socially strictly preferred to $y$ when everyone in $G$ strictly prefers $x$ to $y$, regardless of the preferences of the other individuals. The proof of Arrow's Theorem proceeds by first showing that if a group $G$ is almost decisive for some pair of distinct alternatives, then it is decisive for all pairs of alternatives, in which case $G$ is said to be decisive. Next, it is shown that if any group of two or more individuals is decisive, then it contains a smaller decisive group. WP ensures that the set of all individuals is decisive. Hence, by the previous step in this argument, some individual is decisive; i.e., there is a dictator. At various steps in this argument, it is necessary to construct profiles with particular configurations of preferences on pairs or triples of alternatives. On economic domains, these profiles may not exist.

A linear ordering on $X$ is an ordering in which no distinct alternatives are indifferent to each other. The set of linear orderings of $X$ is $\mathcal{L}$.

Unrestricted Linear Preference Domain. $\mathcal{D}=\mathcal{L}^{n}$.

Straightforward modifications to the standard proofs of Arrow's Theorem show that $\mathcal{L}^{n}$ is also an Arrow-inconsistent domain.

\section{Single-peaked preferences}

In this section, we suppose that the set of alternatives is one-dimensional and that preferences are single-peaked. The analysis of social choice with

\footnotetext{
${ }^{6}$ This version of Arrow's Theorem may be found in Chapter VIII of Arrow (1963). The original statement of the theorem in Arrow (1951) contains a minor error, a fact discovered by Blau (1957). Blau provided a correct statement of the impossibility theorem, but, as noted by Arrow (1963), Blau's version of the theorem contains a redundant axiom.
} 
single-peaked preferences was first considered by Black (1948) in his pioneering work on majority rule. Black's research predates that of Arrow and is non-axiomatic. Subsequently, Moulin (1980) initiated the axiomatic study of strategy-proof social choice for this preference domain. We show how Moulin's generalized median voting schemes can be used to establish the Arrow-consistency of the domain of single-peaked preference profiles. We also discuss the recent characterization by Ehlers and Storcken (2002) of all the social welfare functions that satisfy IIA and WP on this domain.

For concreteness, we assume that $X$ is a nondegenerate interval of $\mathbb{R}$. $X$ can be given a variety of interpretations. For example, if $X=\mathbb{R}_{+}$, an alternative can be interpreted as being the quantity of a public good. Alternatively, an alternative can be a political candidate's ideology, as measured on a left-right spectrum. In either case, it is natural to suppose that preferences are single-peaked. A preference $R \in \mathcal{R}$ is single-peaked on $X \subseteq \mathbb{R}$ if there exists a $\beta \in X$ such that $x P y$ whenever $\beta \leq x<y$ or $\beta \geq x>y$. The alternative $\beta$ is the peak of $R$. Let $\mathcal{S}_{P}$ denote the set of all single-peaked preferences and $\mathcal{S}_{P}^{n}$ denote the set of all profiles of single-peaked preferences on $X{ }^{7}$ In this section, we consider the preference domain $\mathcal{S}_{P}^{n}$.

Unrestricted Single-Peaked Preference Domain. $\mathcal{D}=\mathcal{S}_{P}^{n}$.

Let $\mathcal{B}$ denote the set of all binary relations on $X$. The method of majority rule on $\mathcal{D}$ is a mapping $M: \mathcal{D} \rightarrow \mathcal{B}$ that weakly ranks one alternative above another if and only if the former is weakly preferred to the latter by a majority of individuals. Formally, for all $x, y \in X$ and all $\mathbf{R} \in \mathcal{D}$,

$$
x M(\mathbf{R}) y \leftrightarrow\left|\left\{i \in N \mid x R_{i} y\right\}\right| \geq\left|\left\{i \in N \mid y R_{i} x\right\}\right| .
$$

In general, the method of majority rule is not a social welfare function because it does not always result in a social ordering of the alternatives. However, Black (1948) has shown that if preferences are single-peaked and there are an odd number of individuals, then the majority-rule social preference relation is, in fact, an ordering. ${ }^{8}$

\footnotetext{
${ }^{7}$ In the absence of a natural order on the set of alternatives, single-peakedness is best thought of as a restriction on preference profiles. See Arrow (1951) and Austen-Smith and Banks (1999).

${ }^{8}$ See Gaertner $(2001 ; 2002)$ for a discussion of other domain restrictions that ensure that majority rule yields transitive social preferences.
} 
Theorem 2. If $X$ is a nondegenerate interval of $\mathbb{R}$ and $|N|$ is odd, then the method of majority rule is a social welfare function on an unrestricted single-peaked preference domain. ${ }^{9}$

Theorem 2 can be used to help establish Black's median-voter theorem. Black's Theorem shows that, for any profile $\mathbf{R} \in \mathcal{S}_{P}^{n}$, the median of the individual preference peaks is equal to the alternative in $X$ that is top-ranked according to the majority-rule preference relation $M(\mathbf{R})$ if $n$ is odd.

The binary relation $\leq($ resp. $\geq)$ on $X$ declares $x$ to be weakly preferred to $y$ if and only if $x \leq y$ (resp. $x \geq y$ ). If the infimum (resp. supremum) of $X$ is not in $X, \leq$ (resp. $\geq$ ) is not single-peaked on $X$. However, the extensions of these relations to the closure $\bar{X}$ of $X$ are single-peaked..$^{10}$ It is sometimes useful to augment the set of single-peaked preferences with these binary relations. Let $\mathcal{S}_{P}^{*}=\mathcal{S}_{P} \cup\{\leq, \geq\}$. Theorem 2 also holds for the preference domain $\left(\mathcal{S}_{P}^{*}\right)^{n}$.

Example 1 shows that it is possible to satisfy all of the Arrow axioms, with ND strengthened to ANON, on the domain of single-peaked preference profiles. Thus, this preference domain is Arrow-consistent. ${ }^{11}$

Example 1. Let $X$ be a nondegenerate interval of $\mathbb{R}$. A social welfare function $F: \mathcal{S}_{P}^{n} \rightarrow \mathcal{R}$ is a generalized median social welfare function if there exists a profile $\mathbf{R}^{P} \in\left(\mathcal{S}_{P}^{*}\right)^{n-1}$ such that for all $\mathbf{R} \in \mathcal{S}_{P}^{n}, F(\mathbf{R})=M\left(\mathbf{R}, \mathbf{R}^{P}\right)$. That is, we apply the method of majority rule to a profile consisting of the preferences of the $n$ real individuals and the preferences of $n-1$ phantom voters. ${ }^{12}$ The preferences of these phantom voters are fixed. Because there are an odd number of real and phantom individuals, Theorem 2 implies that $F$ is a social welfare function. Generalized median social welfare functions are the social welfare function analogues of a class of social choice functions introduced by Moulin (1980).

The method of majority rule is based on pairwise comparisons, so $F$ satisfies IIA. Because there are more real individuals than phantoms, if all of the

\footnotetext{
${ }^{9}$ See Arrow (1951) for a proof of this theorem. When the number of individuals is even, the social binary relation resulting from majority rule when preferences are single-peaked is reflexive and complete and the corresponding strict preference relation is transitive, but the social indifference relation may be intransitive.

${ }^{10}$ If $X=\mathbb{R}$, its closure is $\mathbb{R} \cup\{-\infty, \infty\}$.

${ }^{11}$ Arrow (1951) has used Theorem 2 to show that the method of majority rule satisfies all of the axioms used in the original version of his theorem on the domain of single-peaked preference profiles when the number of individuals is odd.

${ }^{12}$ This terminology is due to Border and Jordan (1983).
} 
real individuals strictly prefer $x$ to $y$, then a majority of all the individuals, both real and phantom, prefer $x$ to $y$. Hence, $F$ satisfies WP. Permuting preferences has no effect on the number of individuals who rank $x$ above $y$, so $F$ also satisfies ANON.

In Example 1, if $n$ is odd, there are an even number of phantom voters. By choosing $\mathbf{R}^{P}$ so that half of the phantoms have the preference $\leq$ and the other half have the preference $\geq$, the phantom voters just cancel each other out, and so have no influence on the social ranking. With this profile of phantom preferences, the corresponding generalized median social welfare function is simply the ordinary method of majority rule considered by Black.

A nonempty group $G \subseteq N$ is semidecisive for the ordered pair $(x, y) \in X^{2}$ if $x$ is socially weakly preferred to $y$ when everyone in $G$ strictly prefers $x$ to $y$ and everyone else has the opposite strict preference. Suppose that $X$ is a nondegenerate interval of $\mathbb{R}, x, y, z \in X$ with $x<y<z$ or $z<y<x$, and $\emptyset \neq G \subseteq N$. Ehlers and Storcken (2002) have shown that the decisiveness properties described in our sketch of the proof of Arrow's Theorem only hold in a weaker form when preferences are single-peaked. Specifically, they have shown that if a social welfare function with an unrestricted single-peaked preference domain satisfies IIA and WP, then (i) if $G$ is semidecisive for $(x, y)$, then it is decisive for $(x, z)$ and (ii) if $G$ is semidecisive for $(x, z)$, then it is decisive for $(y, z)$. These decisiveness properties are compatible with nondictatorial rules.

As in Example 1, the social welfare functions in the Ehlers-Storcken (2002) characterization theorem use a set of fixed preferences to augment the actual profile of preferences. However, these supplementary preferences are drawn from a slightly more general class of preferences than the class of single-peaked preferences. A preference $R \in \mathcal{R}$ is strictly quasiconcave on an interval $X$ of $\mathbb{R}$ if for any $x, y, z \in X$ with $x<y<z$, either $y P x$ or $y P z$; i.e., there is a single-peaked preference on $X$ that coincides with $R$ on $\{x, y, z\}$. With a strictly quasiconcave preference, there exists a quasi-peak $\beta \in \bar{X}$ such that (i) $x P y$ if either $y<x<\beta$ or $\beta>x>y$ and (ii) if $\beta \in X$, then either $\beta P x$ for all $x<\beta$ or $\beta P x$ for all $x>\beta$. Note that the quasi-peak can be worse than some of the alternatives on one, but not both, sides of it. If both of the conditions in (ii) hold, $R$ is a single-peaked preference.

In the Ehlers-Storcken construction, there is a fixed strictly quasiconcave preference assigned to each possible subset of $N$, including the empty set. For all $S \subseteq N$, let $R^{S}$ denote the preference assigned to the set $S$ and $\beta^{S}$ 
denote its quasi-peak. Let $\mathbf{R}^{V}$ denote the profile of these preferences. The profile $\mathbf{R}^{V}$ is monotone if for all $S, T \subseteq N$ for which $S \subseteq T$ and for all $w, x, y, z \in X$ for which $w<x<y<z$, (i) $x R^{S} y \rightarrow x P^{T} z$, (ii) $x R^{S} z \rightarrow$ $y P^{T} z$, (iii) $y R^{T} x \rightarrow y P^{S} w$, and (iv) $y R^{T} w \rightarrow x P^{S} w$. The profile $\mathbf{R}^{V}$ is strongly monotone if for all $S, T \subseteq N$ for which $S \subseteq T$ and for all $x, y \in X$ for which $x<y$, (i) $x R^{S} y \rightarrow x R^{T} y$ and (ii) $x P^{S} y \rightarrow x P^{T} y$. It is readily verified that $\mathbf{R}^{V}$ is monotone if it is strongly monotone. The profile $\mathbf{R}^{V}$ is symmetric if $R^{S}=R^{T}$ when $|S|=|T|$.

For any pair of distinct alternatives $x, y \in X$, let $\tau_{\{x, y\}}$ be a social welfare function with an unrestricted preference domain for the set of alternatives $\{x, y\}$. A pairwise assignment rule $\tau$ is a family of such functions, one for each pair of distinct alternatives in $X$. The family $\tau$ is symmetric if $\tau_{\{x, y\}}$ is an anonymous social welfare function for all distinct $x, y \in X$. If each $\tau_{\{x, y\}}$ is the method of majority rule for $\{x, y\}$, then $\tau$ is the majority-rule pairwise assignment rule.

Ehlers-Storcken Social Welfare Functions. Given a monotone profile $\mathbf{R}^{V}$ of strictly quasiconcave preferences on $X$ for which $R^{\emptyset}=\geq$ and $R^{N}=\leq$ and given a pairwise assignment rule $\tau$, the Ehlers-Storcken social welfare function $F_{\tau}^{\mathbf{R}^{V}}: \mathcal{S}_{P}^{n} \rightarrow \mathcal{R}$ associated with $\mathbf{R}^{V}$ and $\tau$ is defined as follows. For all $\mathbf{R} \in \mathcal{S}_{P}^{n}, F_{\tau}^{\mathbf{R}^{V}}(\mathbf{R})$ is reflexive. Let $\mathbf{R} \in \mathcal{S}_{P}^{n}$ and consider $x, y \in X$ with $x<y$. Let $S=\left\{i \in N \mid x P_{i} y\right\}$ and $T=\left\{i \in N \mid x I_{i} y\right\}$.

(i) If there exists a $z \in(x, y)$ such that $x R^{S} z$ or there exists a $z \in X$ with $z<x$ such that $z R^{S} y$, then $x F_{\tau}^{\mathbf{R}^{V}}(\mathbf{R}) y$ and $\neg\left(y F_{\tau}^{\mathbf{R}^{V}}(\mathbf{R}) x\right)$.

(ii) If there exist a $z \in(x, y)$ such that $y R^{S \cup T} z$ or there exists a $z \in X$ with $z>y$ such that $z R^{S \cup T} x$, then $y F_{\tau}^{\mathbf{R}^{V}}(\mathbf{R}) x$ and $\neg\left(x F_{\tau}^{\mathbf{R}^{V}}(\mathbf{R}) y\right)$.

(iii) Otherwise, $F_{\tau}^{\mathbf{R}^{V}}$ coincides with $\tau_{\{x, y\}}$ on $\{x, y\} .{ }^{13}$

The social preference assigned to any profile in $\mathcal{S}_{P}^{n}$ by $F_{\tau}^{\mathbf{R}^{V}}$ is constructed using the family of fixed preferences $\left\{R^{S}\right\}_{S \subseteq N}$ and the pairwise assignment rule $\tau$. A striking feature of the social preference $F_{\tau}^{\mathbf{R}^{V}}(\mathbf{R})$ is that it is strictly quasiconcave. See Ehlers and Storcken (2002). It is fairly easy to show that if (i) holds, then $x P^{S} y$, and if (ii) holds, then $y P^{S \cup T} x$. For example, if $x<z<y$ and $x R^{S} z$, because $R^{S}$ is strictly quasiconcave, $\beta^{S} \leq z$, which implies that $z P^{S} y$. Hence, by transitivity, $x P^{S} y$. As a consequence, $F_{\tau}^{\mathbf{R}^{V}}(\mathbf{R})$ coincides with $R^{S}$ on $\{x, y\}$ in case (i) and it coincides with $R^{S \cup T}$ on $\{x, y\}$ in

\footnotetext{
${ }^{13}$ The monotonicity of $\mathbf{R}^{V}$ ensures that it is not possible to satisfy the hypotheses of both (i) and (ii) in the definition of $F_{\tau}^{\mathbf{R}^{V}}$ for a given profile and pair of alternatives.
} 
case (ii). Ehlers and Storcken have shown that when case (iii) holds, (a) $z$ is socially preferred to both $x$ and $y$ for all $z \in(x, y)$ and (b) $x$ and $y$ are both socially preferred to all of the other alternatives. If $F_{\tau}^{\mathbf{R}^{V}}(\mathbf{R})$ is continuous at both $x$ and $y$, this would imply that $x$ and $y$ are socially indifferent. However, discontinuities are permitted, and it is in fact possible to arbitrarily rank $x$ and $y$ in case (iii) while preserving the strict quasiconcavity of $F_{\tau}^{\mathbf{R}^{V}}(\mathbf{R})$. This is why any pairwise assignment rule $\tau$ can be used to order $x$ and $y$ in this case. Note that in order to satisfy IIA, $\tau$ can only depend on the individual rankings of $x$ and $y$.

Theorem 3 is the Ehlers-Storcken characterization theorem for the domain of single-peaked preferences.

Theorem 3. If $X$ is a nondegenerate interval of $\mathbb{R}$, then a social welfare function with an unrestricted single-peaked preference domain satisfies IIA and WP if and only if it is an Ehlers-Storcken social welfare function.

By construction, $F_{\tau}^{\mathbf{R}^{V}}$ satisfies IIA. To see why WP is satisfied, suppose that $x<y$ and consider a profile in $\mathcal{S}_{P}^{n}$ in which everyone's peak is at $x$. Thus, the set $S$ in the definition of $F_{\tau}^{\mathbf{R}^{V}}$ is $N$. For any $z \in(x, y)$, we have $x P^{N} z$ because $R^{N}=\leq$, so $x$ is socially preferred to $y$ by (i). By IIA, this is the social ranking whenever everyone prefers $x$ to $y$. An analogous argument using (ii) applies when everyone prefers $y$ to $x$. To complete the necessity part of the proof, it must be shown that $F_{\tau}^{\mathbf{R}^{V}}(\mathbf{R})$ is an ordering for all $\mathbf{R} \in \mathcal{S}_{P}^{n}$. The proof that $F_{\tau}^{\mathbf{R}^{V}}(\mathbf{R})$ is transitive is quite lengthy, as is the proof of the sufficiency part of this theorem. See Ehlers and Storcken (2002) for the details.

An Ehlers-Storcken social welfare function satisfies ANON if and only if the profile $\mathbf{R}^{V}$ and the pairwise assignment rule $\tau$ are both symmetric. This observation is used in Theorem 4 to provide sufficient conditions for an Ehlers-Storcken social welfare function to be a generalized median social welfare function.

Theorem 4. If $X$ is a nondegenerate interval of $\mathbb{R}, R^{S} \in \mathcal{S}_{P}^{*}$ for all $S \subseteq N$ with $R^{\emptyset}=\geq$ and $R^{N}=\leq, \mathbf{R}^{V}$ is symmetric and strongly monotone, and $\tau$ is the majority-rule pairwise assignment rule, then $F_{\tau}^{\mathbf{R}^{V}}: \mathcal{S}_{P}^{n} \rightarrow \mathcal{R}$ is a generalized median social welfare function. ${ }^{14}$

\footnotetext{
${ }^{14}$ Theorem 4 is a special case of a theorem in Ehlers and Storcken (2002).
} 
Proof. The symmetry of $\mathbf{R}^{V}$ allows us to identify $\mathbf{R}^{V}$ with a profile of $n+1$ single-peaked preferences $\left(R_{n+1}, \ldots, R_{2 n+1}\right)$, where $R_{n+t}=R^{S}$ if $|S|=t-1$. Consider any $\mathbf{R} \in \mathcal{S}_{P}^{n}$ and any $x, y \in X$ with $x<y$. Note that the majority relations $M\left(\mathbf{R}, R_{n+1}, \ldots, R_{2 n+1}\right)$ and $M\left(\mathbf{R}, R_{n+2}, \ldots, R_{2 n}\right)$ are equivalent because $R_{n+1}=\geq$ and $R_{2 n+1}=\leq$, which cancel each other out. Thus, it is sufficient to show that $M\left(\mathbf{R}, R_{n+1}, \ldots, R_{2 n+1}\right)$ coincides with $F_{\tau}^{\mathbf{R}^{V}}(\mathbf{R})$ on $\{x, y\}$. As above, let $S=\left\{i \in N \mid x P_{i} y\right\}$ and $T=\left\{i \in N \mid x I_{i} y\right\}$.

First, suppose that $x$ is strictly preferred to $y$ according to $F_{\tau}^{\mathbf{R}^{V}}(\mathbf{R})$. This preference is only compatible with cases (i) and (iii) in the definition of $F_{\tau}^{\mathbf{R}^{V}}$. In the latter case, the conclusion follows directly from the assumption that $\tau$ is the majority-rule pairwise assignment rule. In the former case, we must have $x P^{S} y$; i.e., $x P_{n+|S|+1} y$. Strong monotonicity then implies that $x P_{n+|T|+1} y$ for all $T \supseteq S$. Thus, in the profile $\left(\mathbf{R}, R_{n+1}, \ldots, R_{2 n+1}\right)$, the individuals in $S$ and the phantom individuals in $\{n+|S|+1, \ldots, 2 n+1\}$ all strictly prefer $x$ to $y$. Hence, $x$ is strictly preferred to $y$ by at least $n+1$ individuals, which is a strict majority.

Second, suppose that $y$ is strictly preferred to $x$ according to $F_{\tau}^{\mathbf{R}^{V}}(\mathbf{R})$, which is only consistent with cases (ii) and (iii). As above, case (iii) is trivial, so suppose that (ii) applies, which implies that $y P_{n+|S \cup T|+1} x$. It then follows from strong monotonicity that $y P_{n+|U|+1} x$ for all $U \subseteq S \cup T$. Thus, in the profile $\left(\mathbf{R}, R_{n+1}, \ldots, R_{2 n+1}\right)$, the individuals in $N \backslash\{S \cup T\}$ and in $\{n+1, \ldots, n+|S \cup T|+1\}$ strictly prefer $y$ to $x$, which is a strict majority.

If $x$ is indifferent to $y$ according to $F_{\tau}^{\mathbf{R}^{V}}(\mathbf{R})$, case (iii) must apply, and the conclusion is trivial.

\section{Saturating preference domains}

Kalai, Muller, and Satterthwaite (1979) have identified a sufficient condition for a preference domain to be Arrow-inconsistent when everyone has the same set of admissible preferences. Domains satisfying their sufficient condition are called saturating. This structural property of a preference domain is satisfied in many economic and political applications. The methodology used to show that a saturating domain is Arrow-inconsistent is known as the local approach. In this section, we describe the local approach and present some general results about saturating preference domains. 


\subsection{Preliminaries}

As a maintained assumption, we assume throughout this section that the set

of admissible preference orderings for any one individual is independent of the preferences of the other individuals. Thus, the preference domain can be expressed as the product of individual preference domains $\mathcal{D}_{i} \subseteq \mathcal{R}, i \in N$.

Cartesian Preference Domain. $\mathcal{D}=\prod_{i=1}^{n} \mathcal{D}_{i}$, where $\mathcal{D}_{i} \subseteq \mathcal{R}$ for all $i \in N$.

If each person has the same set of admissible preferences, the preference domain is common.

Common Preference Domain. $\mathcal{D}$ is a Cartesian preference domain with $\mathcal{D}_{i}=$ $\mathcal{D}_{j}$ for all $i, j \in N$.

When the preference domain is common, we let $\mathcal{D}_{*}$ denote the common individual preference domain. For a social welfare function $F$ on a common preference domain, although each person's preferences are required to be in $\mathcal{D}_{*}$, the social preferences are merely required to be in $\mathcal{R}$.

Before considering further restrictions on the preference domain, we need to introduce some additional notation. For any subset $A$ of $X,\left.\mathcal{R}\right|_{A}$ denotes the restriction of $\mathcal{R}$ to $A,\left.\mathcal{D}\right|_{A}$ denotes the restriction of $\mathcal{D}$ to $A$, and $\left.F\right|_{A}$ denotes the restriction of $F$ to $A$. More precisely, $\left.F\right|_{A}$ is the social welfare function with domain $\mathcal{D}$ and range $\left.\mathcal{R}\right|_{A}$ defined by $\left.F\right|_{A}(\mathbf{R})=\left.F(\mathbf{R})\right|_{A}$.

In the theorems and examples we consider, it is necessary to determine how rich the preference domain is when restricted to certain subsets of $X$. The following kinds of sets of alternatives are used extensively.

Trivial Subset. A subset $A$ of $X$ is trivial with respect to $\mathcal{D}_{i}$ if $\left|\mathcal{D}_{i}\right|_{A} \mid=1$. The set $A$ is trivial with respect to $\mathcal{D}$ if there is some $i \in N$ such that $A$ is trivial with respect to $\mathcal{D}_{i}$.

A subset is trivial (with respect to $\mathcal{D}$ ) if there is some individual who has only one admissible preference ordering over this set of alternatives. A trivial pair is a trivial subset containing only two alternatives. Sets which are not trivial are called nontrivial. Note that a nontrivial set with respect to $\mathcal{D}$ must be nontrivial for all individuals.

Free Subset. A subset $A$ of $X$ is free with respect to $\mathcal{D}_{i}$ if $\left.\mathcal{D}_{i}\right|_{A}=\left.\mathcal{R}\right|_{A}$. The set $A$ is free with respect to $\mathcal{D}$ if it is free with respect to $\mathcal{D}_{i}$ for all $i \in N$. 
A subset is free (with respect to $\mathcal{D}$ ) if everyone's preferences are unrestricted on this set of alternatives. A free triple is a free subset containing three alternatives. A free subset (containing more than one alternative) is obviously nontrivial. However, a nontrivial set need not be free.

Strong Connection. Two pairs $A$ and $B$ contained in $X$ are strongly connected with respect to $\mathcal{D}_{i}$ (resp. $\mathcal{D}$ ) if $A \cup B$ is a free triple with respect to $\mathcal{D}_{i}(\operatorname{resp} . \mathcal{D})$.

Connection. Two pairs $A$ and $B$ contained in $X$ are connected with respect to $\mathcal{D}_{i}$ (resp. $\left.\mathcal{D}\right)$ if there exists a finite sequence of pairs contained in $X$, $A_{1}, \ldots, A_{r}$, with $A_{1}=A$ and $A_{r}=B$ such that $A_{j}$ and $A_{j+1}$ are strongly connected with respect to $\mathcal{D}_{i}$ (resp. $\mathcal{D}$ ) for all $j=1, \ldots, r-1$.

In the subsequent discussion, when we refer to two pairs as being strongly connected or connected, we are implicitly assuming that this is relative to $\mathcal{D}$. Two pairs that are strongly connected have exactly one alternative in common and the preference domain is unrestricted on their union. If two pairs of alternatives are connected, the preference domain need not be unrestricted on their union; it is only necessary that there exist a way to link the pairs together so that each adjacent pair in the chain is strongly connected. In general, two pairs $A$ and $B$ may be connected with respect to $\mathcal{D}_{i}$ for all $i \in N$ without being connected because there is not a single sequence of pairs that connects the pairs $A$ and $B$ in all of the individual preference domains $\mathcal{D}_{i}$. That is, it may only be possible to connect $A$ and $B$ in $i$ and $j$ 's preference domains using different intermediate pairs of alternatives. However, if there is a common preference domain, because the preference domain is Cartesian, any pairs that are connected (strongly connected) for any individual are obviously connected (strongly connected) for $\mathcal{D}$.

For any $A$ of $X,\left.F\right|_{A}$ is a function of $\left.\mathcal{D}\right|_{A}$ if IIA is satisfied. This insight plays a central role in the results discussed in Part I. It implies that the structure of a social welfare function on $A$ just depends on the restriction of the preference domain to $A$. In particular, if $F$ satisfies IIA and WP and if $\left.\mathcal{D}\right|_{A}=\left.\mathcal{R}^{n}\right|_{A}$ and $|A| \geq 3$, then Theorem 1 (Arrow's Theorem) implies that $\left.F\right|_{A}$ is dictatorial; i.e., there exists a dictator on $A$. Naturally, (when IIA and WP are satisfied) we may use the same line of reasoning for any other set of alternatives $B$ to conclude that there is a dictator on $B$ if $\left.\mathcal{D}\right|_{B}=\left.\mathcal{R}^{n}\right|_{B}$ and $|B| \geq 3$. However, in general, the dictator on $B$ need not be the same as 
the dictator on $A$. The following example, which appears in Fishburn (1976) and in Kalai, Muller, and Satterthwaite (1979), illustrates this point.

Example 2. Let $N=\{1,2\}$ and $X=\left\{x_{1}, x_{2}, x_{3}, x_{4}, x_{5}, x_{6}\right\}$. Let $\mathcal{D}$ be a common preference domain with $\mathcal{D}_{*}=\left\{R \in \mathcal{R} \mid x_{i} P x_{j}\right.$ for all $i=1,2,3$ and all $j=4,5,6\}$. In this example, $\left\{x_{1}, x_{2}, x_{3}\right\}$ and $\left\{x_{4}, x_{5}, x_{6}\right\}$ are free triples. Hence, if $F$ is a social welfare function on $\mathcal{D}$ satisfying IIA and WP, there must exist a dictator on each of these free triples. Define the social welfare function $F$ by setting, for all $\mathbf{R} \in \mathcal{D}$,

(i) $x R y \leftrightarrow x R_{1} y$ for $x, y \in\left\{x_{1}, x_{2}, x_{3}\right\}$,

(ii) $x R y \leftrightarrow x R_{2} y$ for $x, y \in\left\{x_{4}, x_{5}, x_{6}\right\}$,

(iii) $x P y$ if $x \in\left\{x_{1}, x_{2}, x_{3}\right\}$ and $y \in\left\{x_{4}, x_{5}, x_{6}\right\}$.

This is a well-defined social welfare function because, for all profiles in the domain, the social preference is an ordering. For example, if $x \in\left\{x_{1}, x_{2}, x_{3}\right\}$ and $y, z \in\left\{x_{4}, x_{5}, x_{6}\right\}$, by (iii) we must have both $x P y$ and $x P z$, which ensures that restricted to $\{x, y, z\}, R$ is an ordering regardless of how $y$ and $z$ are ranked. $F$ satisfies IIA and WP, but it is not dictatorial.

The following example demonstrates that an Arrow-consistent domain can be both a superset and subset of Arrow-inconsistent domains.

Example 3. Suppose that $|X| \geq 3$. Let $R^{*}$ denote the ordering on $X$ in which all alternatives are indifferent to each other. Bordes and Le Breton (1990b) have shown that the preference domain $\left(\mathcal{L} \cup\left\{R^{*}\right\}\right)^{n}$ is Arrowconsistent. This domain contains no free triples. Note that $\left(\mathcal{L} \cup\left\{R^{*}\right\}\right)^{n}$ contains $\mathcal{L}^{n}$ and is contained in $\mathcal{R}^{n}$, both of which are Arrow-inconsistent. ${ }^{15}$

An example of a social welfare function $F$ that satisfies all of the Arrow axioms on this domain can be constructed as follows. For all $\mathbf{R} \in \mathcal{D}$ for which $R_{i} \neq R^{*}$ for all $i \in N$, let $F(\mathbf{R})=R_{1}$. For any other $\mathbf{R} \in \mathcal{D}$, let $F(\mathbf{R})=R_{2}$. $F$ clearly satisfies WP and ND. If $\mathbf{R}^{1}$ and $\mathbf{R}^{2}$ coincide on $\{x, y\}$, then either both profiles are in $\mathcal{L}^{n}$ or someone has the preference $R^{*}$ in both profiles. Hence, $F$ satisfies IIA.

\footnotetext{
${ }^{15}$ Kelly (1994) has characterized all the Arrow-inconsistent Cartesian preference domains $\mathcal{D}$ for which $\mathcal{L}^{n} \subseteq \mathcal{D} \subseteq \mathcal{R}^{n}$. A domain of linear preference profiles $\mathcal{D}$ is super-Arrovian if $\mathcal{D}$ is Arrow-inconsistent and every domain $\mathcal{D}^{\prime}$ with $\mathcal{D} \subseteq \mathcal{D}^{\prime} \subseteq \mathcal{L}^{n}$ is also Arrow-inconsistent. Fishburn and Kelly (1997) have provided a characterization of the Arrow-inconsistent domains that are also super-Arrovian.
} 


\subsection{The Kalai-Muller-Satterthwaite theorem}

There is no dictator in Example 2 because no pair of alternatives from either of the free triples is connected to any of the pairs contained in the other free triple. Connectedness is a common feature of the preference domains found in a number of economic and political models. In this section, we present the Kalai-Muller-Satterthwaite Theorem, which shows that if all the nontrivial pairs are connected, then the preference domain is Arrow-inconsistent.

A preference domain is saturating when all nontrivial pairs are connected and there are at least two nontrivial pairs.

Saturating Preference Domain. An individual preference domain $\mathcal{D}_{i}$ (resp. a preference domain $\mathcal{D}$ ) is saturating if (a) there exist at least two nontrivial pairs with respect to $\mathcal{D}_{i}$ (resp. $\mathcal{D}$ ) and (b) any two nontrivial pairs are connected with respect to $\mathcal{D}_{i}($ resp. $\mathcal{D})$.

For any saturating preference domain, any nontrivial pair must belong to a free triple. Hence, it is a free pair. The definition of a saturating preference domain introduced by Kalai, Muller, and Satterthwaite (1979) assumes that the preference domain is common. The more general definition presented here first appeared in Le Breton and Weymark (1996). In generalizing the Kalai-Muller-Satterthwaite definition to an arbitrary Cartesian preference domain, all nontrivial pairs are required to be connected, rather than merely requiring each individual preference domain to be saturating. In general, the Le Breton-Weymark definition of a saturating preference domain is not equivalent to the requirement that each of the individual preference domains is saturating; as noted earlier, in the latter case, nontrivial pairs for different individuals may be connected using different sequences of pairs of alternatives. However, if there is a common preference domain, then the definition of a saturating preference domain given here is obviously equivalent to requiring the common individual preference domain $\mathcal{D}_{*}$ to be saturating.

A preference domain satisfies the free triple property if every triple of distinct alternatives is free. If every triple is free, then every pair is nontrivial. Further, any two pairs are connected. For example, $\{x, y\}$ and $\{u, v\}$ are connected by the pair $\{y, u\}$ if $u \notin\{x, y\}$. Hence, any preference domain that satisfies the free triple property is saturating. In particular, an unrestricted preference domain has the free triple property, and so must be saturating. However, a preference domain can be saturating without satisfying the free 
triple property, as Example 4 demonstrates. ${ }^{16}$

Example 4. Let $N=\{1,2\}$ and $X=\left\{x_{1}, x_{2}, x_{3}, x_{4}\right\}$. Let $\mathcal{D}$ be a common preference domain with $\mathcal{D}_{*}=\left\{R \in \mathcal{R} \mid x_{i} P x_{4}\right.$ for all $\left.i=1,2,3\right\}$. In this example, $\left\{x_{1}, x_{2}, x_{3}\right\}$ is a free triple. Any pair containing $x_{4}$ is trivial.

Kalai, Muller, and Satterthwaite (1979) have established the following generalization of Arrow's Theorem.

Theorem 5. If a social welfare function with a common saturating preference domain satisfies IIA and WP, then it is dictatorial.

Proof. By the definition of a saturating preference domain, there are at least two nontrivial pairs. Let $\{x, y\}$ and $\{u, v\}$ be any two such pairs of alternatives. By the definition of a saturating preference domain, these pairs are connected; i.e., there exists a finite sequence of pairs of alternatives, say $\left\{x_{1}, x_{2}\right\},\left\{x_{2}, x_{3}\right\}, \ldots,\left\{x_{r-1}, x_{r}\right\}$ such that $\left\{x, y, x_{1}\right\},\left\{y, x_{1}, x_{2}\right\}$, $\left\{x_{1}, x_{2}, x_{3}\right\}, \ldots,\left\{x_{r-1}, x_{r}, u\right\}$, and $\left\{x_{r}, u, v\right\}$ are free triples.

By Arrow's Theorem (Theorem 1), there is a dictator on each of these free triples. Consider the first two free triples in this sequence. They have the pair $\left\{y, x_{1}\right\}$ in common. As a consequence, the dictator must be the same for both of these two free triples. By a similar reasoning, we can conclude that each adjacent pair of free triples in the sequence has a common dictator. Thus, there is a dictator on the set $\left\{x, y, x_{1}, \ldots, x_{r}, u, v\right\}$. Any other pair of nontrivial alternatives must be connected to $\{x, y\}$ (and to $\{u, v\}$ ). It then follows from the preceding argument that there is a single dictator, say $d$, on the set of all nontrivial pairs. Because there is a common preference domain, everyone has the same preferences on any trivial pair. WP implies that $d$ is a dictator on the trivial pairs. Thus, $d$ dictates on all of $X$.

Variants of this proof have been used extensively to establish Arrovian impossibility theorems on economic and political domains. This method of proof originated with Kalai, Muller, and Satterthwaite (1979) and is now known as the local approach. The reason for this terminology is that the proof consists of first showing that there is a local dictator on a free triple of alternatives and then showing that this local dictator can be transformed into a global dictator (on the nontrivial pairs) by the connectedness implied by the assumptions on the preference domain.

\footnotetext{
${ }^{16}$ The statements in this paragraph are also valid for individual preference domains.
} 
An implication of the Kalai-Muller-Satterthwaite Theorem is that a common preference domain must not be saturating if it is Arrow-consistent. This is the case for the domain of single-peaked preference profiles $\mathcal{S}_{P}^{n}$ considered in Section 3. Consider any three points $x, y, z \in \mathbb{R}$ with $x<y<z$. With a single-peaked preference, it is not possible to rank $x$ first, $z$ second, and $y$ third. Hence, there are no free triples and, therefore, $\mathcal{S}_{P}^{n}$ is not saturating.

\subsection{Further properties of saturating preference domains}

The Kalai-Muller-Satterthwaite Theorem shows that a sufficient condition for a preference domain to be Arrow-inconsistent is that it is common and saturating. In this section, we investigate the robustness of this result.

Example 5 demonstrates that a common preference domain need not be saturating in order for it to be Arrow-inconsistent.

Example 5. Let $N=\{1,2\}$ and $X=\left\{x_{1}, x_{2}, x_{3}, x_{4}\right\}$. Let $\mathcal{D}$ be a common preference domain with $\mathcal{D}_{*}=\left\{R \in \mathcal{R} \mid x_{1} I x_{4}\right.$ or $\left.x_{1} P x_{2} P x_{4}\right\}$. All of the pairs are nontrivial, but the pair $\left\{x_{1}, x_{4}\right\}$ is not connected to any other pair, so the preference domain is not saturating. Suppose that $F$ is a social welfare function on $\mathcal{D}$ satisfying IIA and WP. It follows from the fact that $\left\{x_{1}, x_{2}, x_{3}\right\}$ and $\left\{x_{2}, x_{3}, x_{4}\right\}$ are free triples with the two elements $\left\{x_{2}, x_{3}\right\}$ in common that there is a dictator $d$ on every pair with the possible exception of $\left\{x_{1}, x_{4}\right\}$. On this preference domain, for any profile $\mathbf{R}$ with $x_{1} P_{d} x_{4}$, it is also the case that $x_{1} P_{d} x_{2}$ and $x_{2} P_{d} x_{4}$. We have already established that we must have $x_{1} P x_{2}$ and $x_{2} P x_{4}$ for such a profile. By the transitivity of social preference, it follows that $x_{1} P x_{4}$; i.e., $d$ is a dictator. Hence, $\mathcal{D}$ is Arrow-inconsistent.

Both Arrow's Theorem (Theorem 1) and the Kalai-Muller-Satterthwaite Theorem (Theorem 5) assume that the preference domain is not only saturating, but also that it is common. Example 6 shows that the common preference domain assumption is essential for these results; i.e., there exist saturating preference domains that are Arrow-consistent.

Example 6. Let $N=\{1,2\}, X=\left\{x_{1}, x_{2}, x_{3}, x_{4}\right\}, \mathcal{D}_{1}=\left\{R_{1} \in \mathcal{R} \mid x_{4} P_{1} x_{i}\right.$ for $i=1,2,3\}$, and $\mathcal{D}_{2}=\left\{R_{2} \in \mathcal{R} \mid x_{i} P_{2} x_{4}\right.$ for $\left.i=1,2,3\right\}$. The social welfare function defined by setting $x R y$ if and only if $x R_{1} y$ when $x, y \in\left\{x_{1}, x_{2}, x_{3}\right\}$ and $x P x_{4}$ when $x \in\left\{x_{1}, x_{2}, x_{3}\right\}$ satisfies IIA, WP, and ND. The preference domain $\mathcal{D}$ is saturating, but it is not common. 
In this example, preferences are unrestricted on the set $A=\left\{x_{1}, x_{2}, x_{3}\right\}$, and any pair of alternatives in this set is nontrivial. All other pairs, i.e., pairs formed with $x_{4}$ as one of the alternatives, are trivial. On the trivial pairs, the two individuals have opposite strict preferences. The social welfare function makes person one a dictator on $A$ and person two a dictator on all of the trivial pairs. For example, person two is a dictator on $B=\left\{x_{3}, x_{4}\right\}$. Although there are "local" dictators on $A$ and $B$ and these two sets overlap, because the intersection of these sets only contains a single alternative, there does not have to be a single dictator on their union. For the local approach to apply, the two sets must have at least two elements in common. ${ }^{17}$

Although the preference domain in Example 6 is Arrow-consistent, the social welfare function used to show the consistency is not very appealing because on every pair of alternatives, someone is a dictator. Furthermore, the same person is the dictator on every nontrivial pair. Theorem 6 demonstrates that the latter property is a general feature of saturating preference domains.

Theorem 6. If a social welfare function with a saturating preference domain satisfies IIA and WP, then there is an individual $d \in N$ who is a dictator on every nontrivial pair.

Proof. Inspection of the proof of Theorem 5 reveals that the part of that proof dealing with nontrivial pairs makes no use of the assumption that the preference domain is common. ${ }^{18}$

As we shall see in the next section, many of the commonly used economic and political preference domains are saturating when alternatives are purely public. Unfortunately, Theorem 5 shows that all common saturating preference domains are Arrow-inconsistent. Furthermore, if the preference domain is both saturating and Arrow-consistent, Theorem 6 informs us that the social welfare function is dictatorial on every nontrivial pair of alternatives.

\footnotetext{
${ }^{17}$ Example 6 is similar to the example used by Blau (1957) to show that the statement of Arrow's Theorem in Arrow (1951) is incorrect.

${ }^{18}$ Note that the assumption in Theorem 6 that the preference domain is saturating cannot be replaced with the assumption that each person's individual preference domain is saturating, as we need to have nontrivial pairs connected by a common sequence of intermediate alternatives for our proof to be valid.
} 


\section{Examples of saturating preference domains}

For a common preference domain, the Kalai-Muller-Satterthwaite Theorem (Theorem 5) tells us that the domain is Arrow-inconsistent if it is saturating. There are three main steps involved in showing that a preference domain is saturating. First, the nontrivial pairs are identified. Second, the free triples are identified. Finally, it must be determined how to connect the nontrivial pairs with free triples. ${ }^{19}$ In practice, this last step is often the hardest, but in many interesting applications it is still relatively simple. In this section, we illustrate this procedure with some examples of common saturating preference domains. In these examples, the universal set of alternatives $X$ is a subset of the $m$-dimensional Euclidean space $\mathbb{R}^{m} \cdot{ }^{20}$

Example 7. In this example, we consider the domain of continuous preferences on any connected subset $X$ of $\mathbb{R}^{m}$. A preference ordering $R \in \mathcal{R}$ is continuous if for all $x \in X$, the sets $\{y \in X \mid y R x\}$ and $\{y \in X \mid x R y\}$ are both closed. The preference domain consisting of all profiles of continuous preference orderings has the free triple property and, hence, is saturating because continuity places no restriction on how a triple of alternatives can be ordered. For example, suppose that we want to find a continuous preference $R$ for which $x P y P z$. We can find a neighbourhood $N(x)$ of $x$ in $X$ such that $y, z \notin N(x)$. By Urysohn's Theorem [see Munkres (1975)], there exists a continuous function $V^{1}: X \rightarrow \mathbb{R}$ for which (i) $V^{1}(x)=2 \geq V^{1}(w)$ for all $w \in X$ and (ii) $V^{1}(w)=0$ for all $w \notin N(x)$. Similarly, there exists a neighbourhood $N(y)$ of $y$ in $X$ such that $x, z \notin N(y)$ and a continuous function $V^{2}: X \rightarrow \mathbb{R}$ for which (i) $V^{2}(y)=1 \geq V^{2}(w)$ for all $w \in X$ and (ii) $V^{2}(w)=0$ for all $w \notin N(y)$. The function $U=V^{1}+V^{2}$ is a utility function representing a continuous preference $R$ on $X$ for which $x P y P z .^{21}$

Example 8. We now consider the domain of classic economic preferences when there are two or more divisible public goods. Before presenting the details of this example, we need a few more definitions. Suppose $X$ is a connected subset of $\mathbb{R}^{m}$. A preference ordering $R \in \mathcal{R}$ is monotone (resp.

\footnotetext{
${ }^{19}$ This is the procedure used earlier to show that a preference domain is saturating if every triple is free.

${ }^{20}$ We let $\mathbb{R}_{+}^{m}$ denote the nonnegative orthant, $\mathbb{R}_{++}^{m}$ denote the positive orthant, and $0_{m}$ denote the origin in $\mathbb{R}^{m}$.

${ }^{21}$ For a rigorous proof that this domain has the free triple property, see Campbell (1992b, Chapter 8).
} 
strictly monotone) if $x P y$ for all $x, y \in X$ such that $x \gg y$ (resp. $x>y){ }^{22} \mathrm{~A}$ preference ordering $R \in \mathcal{R}$ is convex (resp. strictly convex) if for all distinct $x, y \in X$ and all $\lambda \in(0,1), x R y$ implies that $[\lambda x+(1-\lambda) y] R y$ (resp. $[\lambda x+(1-\lambda) y] P y)$.

In our example, $X=\mathbb{R}_{+}^{m}$ with $m \geq 2$ and individuals can have any continuous, strictly monotonic, convex preference ordering on $X{ }^{23}$

Classical Public Goods Preference Domain. $\mathcal{D}$ is the set of all profiles of continuous, strictly monotonic, convex preference orderings on $X$.

For future reference, let $\mathcal{C}_{p u}$ denote the preference domain for this example. An alternative can be interpreted as being a vector of $m$ public goods, with the individual preferences assumed to satisfy the usual regularity conditions found in microeconomic theory. Kalai, Muller, and Satterthwaite (1979) have shown that this preference domain is saturating. We sketch a proof for the case of $m=2 .^{24}$

Step 1. We first identify the nontrivial pairs. Strict monotonicity of the preferences implies that any pair of alternatives $\{x, y\}$ with $x>y$ must be a trivial pair. It is easy to verify that if neither $x>y$ nor $y>x$ holds, then $\{x, y\}$ is nontrivial. Simple geometry can be used to confirm this fact. For example, the pair $\{x, y\}$ shown in Figure 1 is nontrivial. ${ }^{25}$

Step 2. We now identify the free triples. Consider the nontrivial pair $\{x, y\}$ shown in Figure 1. It is easy to check that $\{x, y, z\}$ is a free triple if and only if $z$ is in one of the open regions marked III, VI, and IX. (If $x$ is on the vertical axis, there is no region III. Analogously, if $y$ is on the horizontal axis, there is no region IX.) For example, consider the free triple $A=\{x, y, z\}$ shown in Figure 2. There are thirteen (weak) orderings of $A$. Suppose, for concreteness, we want to confirm that there is an admissible preference ordering with $x P y$ and $y P z$. In this case, using a preference ordering with indifference curves similar to those shown in Figure 2 will do.

\footnotetext{
${ }^{22}$ We use the following vector notation: (a) $x \geq y$ means $x_{i} \geq y_{i}$ for all $i=1, \ldots, m$, (b) $x>y$ means $x_{i} \geq y_{i}$ for all $i=1, \ldots, m$ with strict inequality for some $i$, and (c) $x \gg y$ means $x_{i}>y_{i}$ for all $i=1, \ldots, m$.

${ }^{23}$ If $m=1$, there is only one possible individual preference ordering. WP then makes everyone a dictator. ND is clearly not appropriate on such a degenerate preference domain.

${ }^{24}$ The basic structure of the the proof when $m \geq 2$ is similar.

${ }^{25}$ Note that if the individual preference domains include all the continuous, monotone, and convex preference orderings (i.e., strict monotonicity is relaxed to monotonicity), a pair such as $\{v, x\}$ in Figure 1 is no longer trivial.
} 
It is straightforward to check that for any of the other twelve orderings of $A$, there is an ordering $R$ in the domain with $\left.R\right|_{A}$ coinciding with the prespecified ordering of $\{x, y, z\}$. Strict monotonicity prevents any alternative in the regions marked I, II, V, VIII, XI, and XII in Figure 1 from being part of a free triple with $x$ and $y$. Also requiring preferences to be convex precludes any alternative in the regions marked IV, VII, and X from being part of a free triple with $x$ and $y$. For example, consider the alternative $w$ shown in Figure 1 and suppose we want to have $x I y$ and $y P w$. This is obviously impossible with strictly monotonic, convex preferences, so $\{w, x, y\}$ is not a free triple.

Step 3. We now show how to connect nontrivial pairs. Consider the two nontrivial pairs $\{x, y\}$ and $\{u, v\}$ shown in Figure 3. All four of these alternatives have been chosen to be in the positive orthant. We introduce two new alternatives $w$ and $z$, with $w$ lying on the vertical axis and $z$ lying on the horizontal axis. By choosing these points to be sufficiently far from the origin, the preceding argument shows that $\{u, v, z\},\{v, z, w\},\{z, w, x\}$, and $\{w, x, y\}$ are free triples. Thus, by simply considering the two additional alternatives $w$ and $z$, we are able to connect $\{u, v\}$ with $\{x, y\}$ using the sequence of nontrivial pairs $\{u, v\},\{v, z\},\{z, w\},\{w, x\}$, and $\{x, y\}$. When either of the nontrivial pairs $\{x, y\}$ or $\{u, v\}$ has an element on one of the axes, the connection argument is not quite as easy. First, such a nontrivial pair must be connected to a nontrivial pair in the interior of $X$ and then the argument proceeds as above.

In a series of articles summarized in Campbell (1992b; 1996), Campbell has developed an interesting variation of the local approach that can be used to show that economic domains like the one considered in Example 8 are Arrow-inconsistent. His approach may be illustrated using the preference domain of Example 8 with $m=2$, but with $X=\mathbb{R}_{++}^{2}$. A strictly convex downward-sloping curve is the graph of a function $h: \mathbb{R}_{++} \rightarrow \mathbb{R}_{++}$that is continuous, unbounded above, strictly convex, and decreasing. Let $\mathcal{I}$ be the set of all such curves. Each of the curves in $\mathcal{I}$ can be thought of as being a subset of the set of alternatives $X$. It is not difficult to see that for any $C \in \mathcal{I}$, any triple of distinct alternatives in $C$ is a free triple. For example, if the curve $C$ contains the alternatives $x$ and $y$ shown in Figure 1, then any third alternative $z$ in $C$ must lie either in region III, VI, or IX, which makes $\{x, y, z\}$ a free triple. If a social welfare function with this domain satisfies IIA and WP, Arrow's Theorem implies that there is a "local" dictator, say $i(C)$, on $C$. We claim that there exists an $i \in N$ such that $i(C)=i$ for all 
$C \in \mathcal{I}$. Consider two strictly convex downward-sloping curves $C^{1}$ and $C^{2}$ defined by the functions $h^{1}$ and $h^{2}$, respectively. If these curves intersect, then the curve $C^{3}$ defined by taking the supremum of $h^{1}(s)$ and $h^{2}(s)$ for all $s \in \mathbb{R}_{++}$is also in $\mathcal{I}$. Because the curves $C^{1}$ and $C^{3}$ have more than one alternative in common (in fact, they have a continuum of alternatives in common $), i\left(C^{1}\right)=i\left(C^{3}\right)$. Similarly, $i\left(C^{2}\right)=i\left(C^{3}\right)$ and, hence, the dictators on $C^{1}$ and $C^{2}$ are the same. If $C^{1}$ and $C^{2}$ do not intersect, it is possible to find a third curve that intersects them both, and this curve can be used to connect the first two. ${ }^{26}$

Example 9. In this example, a preference ordering $R \in \mathcal{R}$ on a subset $X$ of $\mathbb{R}^{m}$ is linear if there exists a $\pi \in \mathbb{R}^{m}$ such that for all $x, y \in X, x R y$ if and only if $\pi x \geq \pi y \cdot{ }^{27} \mathrm{~A}$ linear preference ordering is continuous and has hyperplanes (linear surfaces) for indifference contours.

The set of alternatives is $X=\mathbb{R}_{+}^{m}$ with $m \geq 3$ and the preference domain is the set of all profiles of continuous, strictly monotonic, and linear preference orderings on $X$. Kalai, Muller, and Satterthwaite (1979) have shown that this preference domain is saturating. We refer the reader to their article for the details. In fact, Kalai, Muller, and Satterthwaite used linear preferences to perform the connection operations in their proof that the preference domain in Example 8 is saturating when $m \geq 3$.

The assumption that $m \geq 3$ is essential for this domain of preferences to be saturating. Kalai, Muller, and Satterthwaite have shown that the preference domain considered in this example is Arrow-consistent when $m=$ 2 and, hence, it is not saturating. ${ }^{28}$ A social welfare function satisfying all of the Arrow axioms on this domain when $m=2$ can be constructed as follows. For each profile of preferences in the domain, the social preference is set equal to the preference of the individual whose indifference curves have the median slope, with ties broken in favour of the smallest median slope if

\footnotetext{
${ }^{26}$ If $X=\mathbb{R}_{+}^{2}$ instead of $\mathbb{R}_{++}^{2}$, the preceding argument does not show that the dictator on the interior of $X$ is a dictator on all of $X$. To extend his argument to all of the nonnegative orthant, Campbell assumed that social preferences are continuous.

${ }^{27}$ These linear preferences should not be confused with the linear preference orderings introduced earlier. Unfortunately, the same name is used for both kinds of preference. Except in this and the following example, a linear preference refers to an ordering for which no two distinct alternatives are indifferent.

${ }^{28}$ With a (strictly) monotone, linear preference ordering, the triple $\{x, y, z\}$ shown in Figure 2 is not free, because it is not possible to have all three elements of the triple indifferent to each other.
} 
there are two medians. ${ }^{29}$

Example 10. In this example, $X$ is the $(m-1)$-dimensional unit simplex $S^{m-1}=\left\{x \in \mathbb{R}_{+}^{m} \mid \sum_{i=1}^{m} x_{i}=1\right\}$. The preference domain is the set of all profiles of linear preference orderings on $X$, where a linear preference is as defined in Example 9. $X$ can be interpreted as being the set of lotteries over $m$ outcomes or prizes; i.e., an alternative $x$ is a probability vector with $x_{j}$ being the probability of obtaining the $j$ th prize. The common individual preference domain can then be interpreted as being the set of expected utility preferences axiomatized by von Neumann and Morgenstern (1947). The vector $\pi$ that characterizes a linear preference ordering is the vector of von Neumann-Morgenstern utilities for the $m$ prizes, where $\pi_{j}$ is the von Neumann-Morgenstern utility of the $j$ th prize. With this interpretation, $x$ is weakly preferred to $y$ if and only if the expected utility of the lottery $x$ is at least as large as the expected utility of the lottery $y$.

For $m \geq 3$, Le Breton (1986) has shown that this preference domain is saturating. We outline the proof for the $m=3$ case. $^{30}$

Step 1. It is obvious that every pair is nontrivial.

Step 2. A triple $\{x, y, z\}$ is free if and only if its three elements are not colinear. For example, $\{x, y, z\}$ in Figure 4 is a free triple. If, for example, we want to find a linear preference ordering that has $z P x$ and $x I y$, all of the indifference curves must be parallel to the dashed line through $x$ and $y$ with $(0,1,0)$ being the most preferred alternative in $X$. It is easy to verify that each of the other possible configurations of preference on $\{x, y, z\}$ can be obtained with a linear preference. ${ }^{31}$ A colinear triple such as $\{u, x, y\}$ in Figure 4 is not free; with linear preferences, if $x I y$, we must also have $u I y$.

Step 3. We now show how to connect nontrivial pairs. Consider the two pairs $\{x, y\}$ and $\{u, v\}$ shown in Figure 4. To make things interesting, we have chosen $u$ so that it is colinear with $x$ and $y$. It is clear that we can always choose two alternatives $w$ and $z$ in $X$ so that each of the following triples

\footnotetext{
${ }^{29}$ Kalai, Muller, and Satterthwaite do not include the tie-breaking rule, so their social welfare function is not well-defined if there are an even number of individuals. Tie-breaking rules other than the one considered above may not satisfy IIA. See Bossert and Weymark (1993) for a detailed discussion of this two-dimensional problem. Kalai, Muller, and Satterthwaite's analysis of this example is based, in part, on Nitzan (1976).

${ }^{30}$ Again, the argument is easily extended to higher dimensions. When $m=2$, each person has only three possible preference orderings.

${ }^{31}$ If we want to have all three elements of the triple $\{x, y, z\}$ in Figure 4 indifferent to each other, then there must be universal indifference over all lotteries in $X$.
} 
consists of alternatives that are not colinear: $\{x, y, z\},\{y, z, w\},\{z, w, u\}$, and $\{w, u, v\}$. Figure 4 illustrates this construction. We connect $\{u, v\}$ with $\{x, y\}$ using the sequence of nontrivial pairs $\{x, y\},\{y, z\},\{z, w\},\{w, u\}$, and $\{u, v\}$. Thus, we are able to connect the two nontrivial pairs $\{x, y\}$ and $\{u, v\}$ using only two additional alternatives, and this is the case even if any of the original alternatives lie on the boundary of $X .^{32}$

Example 11. In this example, we further restrict the domain of classical public goods preferences considered in Example 8 by also assuming that preferences are analytic with no critical points. Recall that $X=\mathbb{R}_{+}^{m}$ with $m \geq 2$ and $\mathcal{C}_{p u}$ is the common individual preference domain in Example 8. A preference $R$ on a connected subset of $\mathbb{R}^{m}$ is analytic if it can be represented by an analytic utility function; i.e., by a utility function that has the property that at any point $x$ in the domain, there is a neighbourhood of $x$ on which the function can be expressed exactly as a Taylor series. A preference $R$ on a connected subset of $\mathbb{R}^{m}$ has no critical point if it can be represented by a utility function whose gradient never vanishes. Let $\mathcal{M}_{c}$ denote the set of all strictly monotone, convex, analytic preferences with no critical points on $\mathbb{R}_{+}^{m}$. $\mathcal{M}_{c}$ is a subset of $\mathcal{C}_{p u}$. Many of the functional forms used for utility functions, such as Cobb-Douglas and CES, are analytic with no critical points, at least on the positive orthant.

Le Breton and Weymark (2002) have shown that the domain $\mathcal{M}_{c}^{n}$ is Arrow-inconsistent. ${ }^{33}$ In fact, this domain is saturating, as we now show. Kannai (1974) has demonstrated that $\mathcal{M}_{c}$ is a dense subset of $\mathcal{C}_{p u}{ }^{34}$ It follows from this fact that the nontrivial pairs and free triples of $\mathcal{M}_{c}$ and $\mathcal{C}_{p u}$ are exactly the same. To see why, let $\{x, y, z\}$ be a free triple with respect to $\mathcal{C}_{p u}$. Let $R \in \mathcal{C}_{p u}$ be such that $x P y P z$. From Kannai's result, we then deduce that there exists a preference $R^{\prime} \in \mathcal{M}_{c}$ such that $x P^{\prime} y P^{\prime} z$. This argument shows that any of six strict orderings on $\{x, y, z\}$ can be obtained

\footnotetext{
${ }^{32}$ Note that preferences are trivially strictly monotonic on a simplex. Thus, the preference domain in this example can be thought of as the restriction of the preference domain in the preceding example (where $X$ is the nonnegative orthant) to a simplex. On the nonnegative orthant, strict monotonicity places considerable structure on the preferences, which helps account for the fact that the set of strictly monotone, linear preferences is saturating when the set of alternatives is the two-dimensional simplex, but it is not saturating when the set of alternatives is the two-dimensional nonnegative orthant.

${ }^{33}$ Le Breton and Weymark assumed that all admissible preferences are strictly convex, not just convex, but this is not essential for their argument.

${ }^{34} A$ is a dense subset of $B$ if $B$ is contained in the closure of $A$.
} 
with a preference in $\mathcal{M}_{c}$. To show that we can also obtain the other seven possible weak orderings, we use the preceding argument in conjunction with the fact that analyticity is preserved by convex combinations. For example, to obtain a preference $R \in \mathcal{M}_{c}$ with $x I y P z$, we first consider $R^{\prime}, R^{\prime \prime} \in \mathcal{M}_{c}$ for which $x P^{\prime} y P^{\prime} z$ and $y P^{\prime} x P^{\prime} z$. Next, let $U^{\prime}$ and $U^{\prime \prime}$ be two analytic utility functions with no critical points representing $R^{\prime}$ and $R^{\prime \prime}$, respectively, and having values in $\mathbb{R}_{+}$. By choosing $\lambda \in[0,1]$ appropriately, the utility function $\lambda U^{\prime}+(1-\lambda) U^{\prime \prime}$ represents a preference $R_{\lambda} \in \mathcal{M}_{c}$ for which $x I_{\lambda} y P_{\lambda} z$. Thus, the free triples of $\mathcal{M}_{c}$ and $\mathcal{C}_{p u}$ coincide. Similar arguments show that the nontrivial pairs coincide as well. Therefore, we can use the connection argument in Example 8 to show that $\mathcal{M}_{c}$ and, hence, $\mathcal{M}_{c}^{n}$ is saturating.

Example 12. In this example, we consider Euclidean spatial preferences. A preference $R \in \mathcal{R}$ on a subset $X$ of $\mathbb{R}^{m}$ is a Euclidean spatial preference if there exists a point $\beta \in X$ such that for all $x, y \in X, x R y$ if and only if $\|x-\beta\| \leq\|y-\beta\|$, where $\|\cdot\|$ denotes the Euclidean norm on $\mathbb{R}^{m}$. ${ }^{35}$ With a Euclidean spatial preference, alternatives are ranked by their Euclidean distance from a point of global satiation $\beta$ known as an bliss or ideal point. A Euclidean spatial preference is analytic because it can be represented by a quadratic function with a critical point at the bliss point. When $m=1$, a Euclidean spatial preference is a continuous single-peaked preference that is symmetric with respect to the bliss point.

Spatial preferences are used extensively by political scientists in formal voting models. In these applications, $X$ is referred to as an issue space. The coordinates of $X$ might, for example, measure the budgets for different categories of public expenditure (police, garbage collection, etc.), with voters assumed to have spatial preferences. Spatial preferences are also found in some economic models of public good provision. In these applications, the presence of a satiation point arises because attention is restricted to allocations satisfying a budget constraint. For an introduction to spatial models, see Austen-Smith and Banks (1999).

For the case in which $X=\mathbb{R}^{m}$ with $m \geq 2$, Border (1984) has shown that Arrow's axioms are inconsistent on the domain of all profiles of Euclidean spatial preferences. Border's proof of this result is long and complicated. ${ }^{36}$

\footnotetext{
${ }^{35}$ More generally, spatial preferences are convex preferences with a point of global satiation. We consider general spatial preferences in Section 16.3.

${ }^{36}$ Redekop (1993c) has established a version of Border's Theorem that does not require the domain to include all profiles of Euclidean spatial preferences.
} 
An alternative way of establishing Border's theorem is to first show that this preference domain is saturating and then use Theorem 5 to conclude that the domain is Arrow-inconsistent. A proof along these lines, due to Le Breton, may be found in Le Breton and Weymark (1996). Both methods of proof take advantage of the fact that $X$ has no boundary points. However, in spatial models, it is more natural to suppose that alternatives have nonnegative components. Accordingly, here we suppose that $X=\mathbb{R}_{+}^{m}$.

Let $\mathcal{E}$ denote the set of all spatial preferences orderings on $\mathbb{R}_{+}^{m}$. Le Breton and Weymark (2002) have shown that the domain $\mathcal{E}^{n}$ is saturating when $m \geq 2 .{ }^{37}$ We provide an alternative proof that $\mathcal{E}^{n}$ is saturating for the case of $m=2 .{ }^{38}$

Step 1. It is obvious that every pair is nontrivial.

Step 2. Campbell (1993) has shown that if a triple $\{x, y, z\}$ is cocircular with a bliss point in the interior of $X$, then $\{x, y, z\}$ is a free triple. We illustrate the argument by showing that there exists an $R \in \mathcal{E}$ such that $x P y P z$. Consider the line segment $[x, y]$ joining $x$ and $y$. Let $H_{x y}$ be the line (hyperplane in higher dimensions) containing the midpoint of $[x, y]$ that is orthogonal to $[x, y]$. In spatial voting models, $H_{x y}$ is referred to as the median line for the alternatives $x$ and $y$. By assumption, $x, y$, and $z$ are equidistant from a point $\beta$ in the interior of $X$. Hence, by construction, $\beta$ is in $H_{x y}$. Let $A^{1}$ and $A^{2}$ be, respectively, the short and the long arcs with extremities $x$ and $y$ on the circle with center $\beta$ and radius $\|x-\beta\| .{ }^{39}$ It is transparent from Figure 5 that if $z \in A^{1}$, then by moving $\beta$ slightly farther from $\frac{(x+y)}{2}$, say to $\beta^{\prime}$, then we obtain a preference $R^{\prime} \in \mathcal{E}$ for which $x I^{\prime} y P^{\prime} z$. Note that this might not be possible if $\beta$ is on the boundary of $X$. To obtain an $R \in \mathcal{E}$ for which $x P y P z$, one merely has to move $\beta^{\prime}$ slightly closer to $x$ than to $y$. On the other hand, if $z \in A^{2}$, the preference $R^{\prime}$ in this argument is obtained by moving the bliss point $\beta$ closer to $\frac{(x+y)}{2}$.

Step 3. We now show that any pair of alternatives is connected to a pair of alternatives whose corresponding median line is parallel to the $45^{\circ}$ line. Let $\{x, y\}$ be an arbitrary pair in $X$ and let $C$ be a circle containing $x$ and $y$ whose bliss point $\beta$ is in the interior of $X$. Let $\left\{L_{\delta}\right\}_{\delta \geq 0}$ be the family of lines that are orthogonal to the $45^{\circ}$ line, parametrized by the point of intersection $(\delta, \delta)$ with the $45^{\circ}$ line. As illustrated in Figure 6 , there exists a $\delta>0$ and

\footnotetext{
${ }^{37}$ Campbell (1993) has established a related result with the additional assumption that social preferences are continuous.

${ }^{38}$ This method of proof is easily generalized to higher dimensions.

${ }^{39}$ If the two arcs are the same length, they can be labelled arbitrarily.
} 
distinct $u, z \in \mathbb{R}_{+}^{2} \backslash\{x, y\}$ such that $u, z \in C \cap L_{\delta}$. Because $u, x, y$, and $z$ are cocircular and $\beta$ is in the interior of $X$, it now follows from Step 2 that $\{x, y, z\}$ and $\{y, z, u\}$ are free triples. Hence, $\{x, y\}$ is connected to $\{z, u\}$. By construction the median line for $\{z, u\}$ is parallel to the $45^{\circ}$ line.

Step 4. Next, we show that if $u, x, y$, and $z$ are in $L_{\delta} \cap X$ for some $\delta>0$, then $\{x, y\}$ and $\{z, u\}$ are connected. Let $\{x, y\}$ be a pair in $L_{\delta} \cap X$ with $\delta>0$. Let $L_{\delta}^{+}=\left\{c=\left(c_{1}, c_{2}\right) \in X \mid c_{1}+c_{2}>2 \delta\right\}$. We claim that there exists an $\varepsilon>0$ such that $\{x, y, c\}$ is a free triple for all $c$ in $[B((0,2 \delta), \varepsilon) \cup B((2 \delta, 0), \varepsilon)] \cap L_{\delta}^{+}$, where $B(\bar{x}, \varepsilon)$ is an open ball of radius $\varepsilon$ centered at $\bar{x}$. The argument is illustrated in Figure 7. For concreteness, consider any $c \in B((0,2 \delta), \varepsilon) \cap L_{\delta}^{+}$. By moving sufficiently far from the origin on $H_{x y}$, we can find a $\beta$ such that $x, y$, and $c$ are equidistant from $\beta$. It then follows from Step 2 that $\{x, y, c\}$ is a free triple.

To conclude the proof of this step, let $\varepsilon$ be small enough for the claim to apply to each of the pairs $\{x, y\},\{y, z\}$, and $\{z, u\}$. Again, for concreteness, consider any $c \in B((0,2 \delta), \varepsilon) \cap L_{\delta}^{+}$. The pairs $\{x, y\}$ and $\{z, u\}$ are connected through the sequence of triples $\{x, y, c\},\{y, c, z\}$, and $\{c, z, u\}$.

Step 5. Suppose that $0<\delta<\delta^{\prime}$. To complete the proof, we show that any pair in $L_{\delta}$ is connected to any pair in $L_{\delta^{\prime}}$. Let $\beta$ be the midpoint of the line segment $\left[(\delta, \delta),\left(\delta^{\prime}, \delta^{\prime}\right)\right]$, as illustrated in Figure 8 . Choose $\varepsilon>0$ sufficiently small that the circle centered at $\beta$ with radius $\|\beta-(\delta, \delta)\|+\varepsilon$ intersects both $L_{\delta} \cap X$ and $L_{\delta^{\prime}} \cap X$ twice. Let $\{x, y\}$ and $\{z, u\}$ be the points of intersection with $L_{\delta}$ and $L_{\delta^{\prime}}$, respectively. By Step 2, $\{x, y, z\}$ and $\{y, z, u\}$ are free triples. Hence, $\{x, y\}$ is connected to $\{z, u\}$. It then follows from Step 4 that any pair in $L_{\delta}$ is connected to any pair in $L_{\delta^{\prime}}{ }^{40}$

Example 13. In this example, we consider continuous separable and continuous, additively separable preferences. Let $m \geq 2$ and suppose that $X$ is a product set $X^{1} \times X^{2} \times \cdots \times X^{m}$, where each $X^{k}$ is a subset of $\mathbb{R}$ with $\left|X^{k}\right| \geq 2 .^{41}$ A preference ordering $R \in \mathcal{R}$ is separable if for all nonempty $\tilde{M} \subset$ $M=\{1, \ldots, m\}$, all $z^{\tilde{M}}, u^{\tilde{M}} \in \Pi_{k \in \tilde{M}} X^{k}$, and all $x^{M \backslash \tilde{M}}, y^{M \backslash \tilde{M}} \in \Pi_{k \in M \backslash \tilde{M}} X^{k}$, $\left(x^{M \backslash \tilde{M}}, z^{\tilde{M}}\right) R\left(y^{M \backslash \tilde{M}}, z^{\tilde{M}}\right)$ if and only if $\left(x^{M \backslash \tilde{M}}, u^{\tilde{M}}\right) R\left(y^{M \backslash \tilde{M}}, u^{\tilde{M}}\right)$. With a sep-

\footnotetext{
${ }^{40}$ This proof makes use of the fact that the set of alternatives is unbounded from above. Ehlers and Storcken (2002) have shown that the domain of Euclidean spatial preferences is Arrow-inconsistent when $X$ is contained in the closure of its interior and the interior of $X$ is nonempty and connected. (As in Example $12, X$ is a subset of $\mathbb{R}^{m}$ with $m \geq 2$.) These assumptions are satisfied if $X$ is a compact subset of $\mathbb{R}_{+}^{m}$ with a nonempty interior.

${ }^{41}$ The following analysis can be easily generalized to the product of $m$ metric spaces.
} 
arable preference, there are orderings on each of the components of $X$ that are independent of the values of the other components. A preference ordering $R$ is additively separable if for all $k \in M$, there exists a function $V^{k}: X^{k} \rightarrow \mathbb{R}$ such that for all $x, y \in X, x R y$ if and only if $\sum_{k=1}^{m} V^{k}\left(x^{k}\right) \geq \sum_{k=1}^{m} V^{k}\left(y^{k}\right)$. In what follows, we denote by $\mathcal{R}_{C S}$ and $\mathcal{R}_{C A S}$, respectively, the set of continuous separable and continuous, additively separable preference orderings on $X$. Clearly, $\mathcal{R}_{C A S}$ is a subset of $\mathcal{R}_{C S}$.

The joint assumption that the set of alternatives is a product set and preferences are separable or additively separable has been considered by many authors from a strategic perspective. See, for example, Le Breton and Weymark (1999). Le Breton and Weymark have considered an example in which proportional taxes can be levied at different rates on the individual endowments of two private goods in order to finance the production of public goods. In their example, $X=[0,1]^{2}$ is the set of possible pairs of tax rates and the individual preference domain is the subset of preferences in $\mathcal{R}_{C A S}$ that can be represented by continuously differentiable utility functions.

We show that $\mathcal{R}_{C A S}^{n}$ is saturating by demonstrating that $\mathcal{R}_{C A S}$ has the free triple property. A fortiori, $\mathcal{R}_{C S}^{n}$ is saturating as well.

For any $x, y \in X$, let $M(x, y)=\left\{k \in M \mid x_{k} \neq y_{k}\right\}$ be the set of components on which $x$ and $y$ differ. Let $\{x, y, z\}$ be a triple of distinct alternatives in $X$. For concreteness, we show that there exists an $R$ in $\mathcal{R}_{C A S}$ such that $x P y P z$. We consider three cases.

Case 1. $M(x, y) \cap M(x, z) \cap M(y, z) \neq \emptyset$. Consider $\bar{k} \in M(x, y) \cap$ $M(x, z) \cap M(y, z)$. As in Example 7, we can construct a continuous function $V^{\bar{k}}: X^{\bar{k}} \rightarrow \mathbb{R}$ for which $V^{\bar{k}}\left(x_{\bar{k}}\right)=2, V^{\bar{k}}\left(y_{\bar{k}}\right)=1$, and $V^{\bar{k}}\left(z_{\bar{k}}\right)=0$. For all other $k \in M$, let $V^{k}\left(w_{k}\right)=0$ for all $w_{k} \in X^{k}$. The function $U=\sum_{k \in M} V^{k}$ is a utility function representing a preference $R \in \mathcal{R}_{C A S}$ for which $x P y P z$.

Case 2. $M(x, y) \cap M(x, z)=\emptyset$. Consider $\bar{k} \in M(x, z)$. By assumption, $x_{\bar{k}}=y_{\bar{k}}$. By Urysohn's Theorem, there exists a continuous function $V^{\bar{k}}: X^{\bar{k}} \rightarrow \mathbb{R}$ for which $V^{\bar{k}}\left(x_{\bar{k}}\right)=V^{\bar{k}}\left(y_{\bar{k}}\right)=2$ and $V^{\bar{k}}\left(z_{\bar{k}}\right)=0$. Next, consider $\tilde{k} \in M(x, y)$. By assumption, $x_{\tilde{k}}=z_{\tilde{k}}$. By Urysohn's Theorem, there exists a continuous function $V^{\tilde{k}}: X^{\tilde{k}} \rightarrow \mathbb{R}$ for which $V^{\tilde{k}}\left(x_{\tilde{k}}\right)=V^{\tilde{k}}\left(z_{\tilde{k}}\right)=1$ and $V^{\tilde{k}}\left(y_{\tilde{k}}\right)=0$. For all other $k \in M$, let $V^{k}\left(w_{k}\right)=0$ for all $w_{k} \in X^{k}$. As in Case 1 , the function $U=\sum_{k \in M} V^{k}$ is a utility function representing a preference $R \in \mathcal{R}_{C A S}$ for which $x P y P z$.

Case 3. $M(x, y) \cap M(x, z) \cap M(y, z)=\emptyset$ and $M(x, y) \cap M(x, z) \neq \emptyset$. For $\bar{k} \in M(x, y) \cap M(x, z)$, Urysohn's Theorem implies that there exists 
a continuous function $V^{\bar{k}}: X^{\bar{k}} \rightarrow \mathbb{R}$ for which $V^{\bar{k}}\left(x_{\bar{k}}\right)=2$ and $V^{\bar{k}}\left(y_{\bar{k}}\right)=$ $V^{\bar{k}}\left(z_{\bar{k}}\right)=0$. For $\tilde{k} \in M(y, z)$, Urysohn's Theorem implies that there exists a continuous function $V^{\tilde{k}}: X^{\tilde{k}} \rightarrow \mathbb{R}$ for which $V^{\tilde{k}}\left(x_{\tilde{k}}\right) \in\{0,1\}$ (this value is 0 if $x_{\tilde{k}}=z_{\tilde{k}}$ and it is 1 if $\left.x_{\tilde{k}}=y_{\tilde{k}}\right), V^{\tilde{k}}\left(y_{\tilde{k}}\right)=1$, and $V^{\tilde{k}}\left(z_{\tilde{k}}\right)=0$. For all other $k \in M$, let $V^{k}\left(w_{k}\right)=0$ for all $w_{k} \in X^{k}$. As in the previous cases, the function $U=\sum_{k \in M} V^{k}$ is a utility function representing a preference $R \in \mathcal{R}_{C A S}$ for which $x P y P z$.

Similar constructions can be used to generate any of the other orderings of $\{x, y, z\}$.

Example 14. In this example, we consider a domain of preferences encountered in the study of income inequality. Let $X=S^{m-1}$ with $m \geq 3$ and suppose that the preference domain consists of all profiles of continuous and strictly Schur-convex orderings of $X$. An ordering $R$ of $S^{m-1}$ is strictly Schurconvex if for all $x, y \in X$, (a) $x R y$ whenever $x=B y$ for some bistochastic matrix $B$ and (b) $x P y$ whenever $x=B y$ for some bistochastic matrix $B$ that is not a permutation matrix. ${ }^{42}$ The set $X$ can be interpreted as being the set of all possible distributions of a unit of income, with the preference domain interpreted as being the set of all profiles of inequality orderings of $X .{ }^{43}$ For symmetric orderings on a simplex, strict Schur-convexity is equivalent to the Pigou-Dalton condition, which requires that an equalizing transfer from a richer to a poorer person results in a preferred distribution. ${ }^{44}$

Le Breton and Trannoy (1987) have shown that this preference domain is saturating. To show that all nontrivial pairs are connected involves introducing a rather large number of intermediate pairs, so we refer the reader to their article for the details of the argument.

In all the examples considered so far in this section, the preference domains are common and saturating. Hence, they are Arrow-inconsistent. We conclude this section with an example of a common preference domain that is Arrow-consistent and, therefore, not saturating. Nevertheless, in this example, there are subsets of alternatives on which the preference domain is

\footnotetext{
${ }^{42} \mathrm{~A}$ square matrix $B$ is bistochastic if it is nonnegative and all of its row and column sums are equal to one. A bistochastic matrix whose entries are all zeroes or ones is a permutation matrix.

${ }^{43}$ The details of this example are unchanged if there is any fixed amount of income $c>0$ to distribute. Note that if $m=2$, each person has only one possible preference ordering.

${ }^{44}$ In this application, a preference order $R$ on $S^{m-1}$ is symmetric if for all $x, y \in X, x I y$ whenever $x=B y$ for some permutation matrix $B$.
} 
saturating, so the local approach can be used to infer quite a bit about the structure of a social welfare function that satisfies the three Arrow axioms.

Example 15. In this example, individuals have probability relations on a finite set of events. We assume that there are $m \geq 4$ states of nature. Each state of nature corresponds to the possible realization of a random variable. An event is a subset of $M=\{1, \ldots, m\}$. The set of alternatives $X$ is the set of all possible events. An ordinal probability is an ordering $R$ on $X$ that can be represented by a probability measure, i..e, by a function $p: M \rightarrow[0,1]$ for which for all $x, y \in X, x R y$ if and only if $\sum_{k \in x} p_{k} \geq \sum_{k \in y} p_{k}$, where $p_{k}=p(k)$. The preference domain is the set of all ordinal probabilities on $X$. This preference domain has been considered by Lainé, Le Breton, and Trannoy (1986) and Weymark (1997).

This example can be restated using the framework of Example 13. Let $X^{*}=\prod_{k \in M} X^{* k}$, where $X^{* k}=\{0,1\}$ for all $k \in M$. The event $x \in X$ can be reinterpreted as being the point $x^{*} \in X^{*}$ for which $x_{k}^{*}=1$ if $k \in x$ and $x_{k}^{*}=0$ if $k \notin x$. With this reinterpretation, the set of ordinal probabilities on $X^{*}$ is a strict subset of $\mathcal{R}_{C A S}$, the set of continuous, additively separable preferences on $X^{*} .{ }^{45}$ The set inclusion is strict because an ordinal probability satisfies monotonicity, among other properties.

In terms of our initial formulation of this example, any triple of events in which no event is a subset of any other event in the triple is free. To see why, let $\{x, y, z\}$ be such a triple of events. Let $w=x \cap y \cap z$. By assumption, $\bar{x}=x \backslash w, \bar{y}=y \backslash w$, and $\bar{z}=z \backslash w$ are all nonempty. By construction, $\bar{x} \cap \bar{y} \cap \bar{z}=\emptyset$. Suppose, for example, that we want to find an ordinal probability $R$ for which $x P y P z$. We consider two cases.

Case 1. $\bar{x} \cap \bar{y} \neq \emptyset$. In this case, we choose a probability measure $p$ on $X$ such that $\sum_{k \in \bar{x} \cap \bar{y}} p_{k}=1-\varepsilon$ and $\sum_{k \in \bar{x} \backslash \bar{y}} p_{k}=\varepsilon$, where $\varepsilon>0$. Note that $p_{i}=0$ for all $i \in w$. Because $\bar{x} \cap \bar{y} \cap \bar{z}=\emptyset$, for sufficiently small $\varepsilon$, this $p$ represents an ordinal probability for which $x P y P z$.

Case 2. $\bar{x} \cap \bar{y}=\emptyset$. By construction, $\bar{x} \backslash \bar{z} \neq \emptyset$ and $\bar{y} \backslash \bar{z} \neq \emptyset$. In this case, we choose $p$ such that $\sum_{k \in \bar{x} \backslash \bar{z}} p_{k}=1-\varepsilon$ and $\sum_{k \in \bar{y} \backslash \bar{z}} p_{k}=\varepsilon$, where $\varepsilon>0$. As in Case 1, when $\varepsilon$ is sufficiently small, $p$ represents an ordinal probability for which $x P y P z$.

All of the other orderings of $\{x, y, z\}$ can be obtained in a similar fashion.

It follows from the preceding discussion that if neither $x \subseteq y$ nor $y \subseteq x$, then $\{x, y\}$ is a free pair. A pair of events $\{x, y\}$ for which $x \subset y$ is neither

\footnotetext{
${ }^{45}$ Continuity is vacuous in this finite setting.
} 
trivial nor free if $x \neq \emptyset$ or $y \neq M$. The reason is that an ordinal probability can rank these events in only one of two ways: $x I y$ or $y P x$. Hence, this preference domain is not saturating. Because $M$ is always more likely than $\emptyset,\{\emptyset, M\}$ is a trivial pair. In fact, this is the only trivial pair.

Let $X(k)$ denote the set of all events containing exactly $k$ states. For $k \notin\{0, m\}$, because no two events in $X(k)$ can be ordered by set inclusion, the restriction of the preference domain to $X(k)$ has the free triple property. Thus, restricted to $X(k)$ for $k \notin\{0, m\}$, the preference domain is saturating. More generally, Lainé, Le Breton, and Trannoy (1986) have shown that any nontrivial pair of events can be connected to any other pair of nontrivial events provided that neither of the event pairs is a single-state event and its complement. ${ }^{46}$ The problem with a pair such as $\{\{1\},\{2,3, \ldots, m\}\}$ is that any other event must be a subset or superset of one of these two events, so the pair cannot be embedded in a free triple.

Now consider a social welfare function defined on the domain of profiles of ordinal probabilities satisfying IIA and WP. Lainé, Le Breton, and Trannoy (1986) have shown that there is an individual $d$ who is a dictator on any ordered pair of events $(x, y)$ for which (i) $|x|>1$ or (ii) $|x|=1$ and $y$ is not the complement of $x{ }^{47}$ We sketch the proof.

In view of the preceding discussion, the argument used to establish the Kalai-Muller-Satterthwaite Theorem shows that there is an individual $d$ who is a dictator on any nontrivial pair except a single-set event and its complement. If $x \subseteq y$, no ordinal probability $R$ can have $x P y$, so $d$ is trivially a dictator on the ordered pair $(x, y)$ when $x \subseteq y$. Because everyone ranks $M$ above $\emptyset$, WP implies that $d$ dictates on the ordered pair $(M, \emptyset)$.

To show that $d$ dictates on the ordered pair $(\{1,2\},\{1\})$, consider a profile $\mathbf{R}$ in which $\{1,2\} P_{d}\{1\}$. Because $\{\{1,2\},\{1,3\}\}$ is a free pair, there exists a profile $\mathbf{R}^{\prime}$ of ordinal probabilities with the properties that (i) $\{1,2\} P_{d}^{\prime}\{1,3\}$, (ii) $\{1,3\} P_{i}^{\prime}\{1\}$ for all $i \in N$, and (iii) for all $i \in N,\{1,2\} P_{i}^{\prime}\{1\}$ if and only if $\{1,2\} P_{i}\{1\}$. Because $d$ is a dictator on $\{\{1,2\},\{1,3\}\}$, we have $\{1,2\} P^{\prime}\{1,3\}$. By WP, we have $\{1,3\} P^{\prime}\{1\}$. Transitivity of $P^{\prime}$ then implies that $\{1,2\} P^{\prime}\{1\}$. Hence, by IIA, $\{1,2\} P\{1\}$ and, therefore, $d$ is a dictator on $(\{1,2\},\{1\})$.

\footnotetext{
${ }^{46}$ This is not true if there are only three states. For example, it is not possible to connect $\{\{1\},\{2\}\}$ with $\{\{1,2\},\{2,3\}\}$ when $m=3$ because there are no free triples containing two events with different cardinalities.

${ }^{47}$ The same conclusion holds when $m=3$ if the social preference relation is also required to be an ordinal probability.
} 
Similar arguments can be used to show that $d$ is a dictator on any of the other ordered pairs not yet considered except a pair of the form $\{\{k\}, M \backslash\{k\}\}$. We cannot utilize a third event, as above, to show that $d$ dictates on this pair because this third event, say $x$, must be a superset of $\{k\}$, and no ordinal probability can rank $\{k\}$ above $x$, as would be required to appeal to WP. ${ }^{48}$

\section{Topological domain restrictions for public goods}

In the preceding two sections, we have shown that saturating preference domains are Arrow-inconsistent and that many domains of economic preferences are saturating. In a series of articles, Redekop (1991; 1993a; 1993c; 1996) has identified topological restrictions on the preference domain that imply that the domain is Arrow-inconsistent. Redekop begins by first restricting the set of admissible preference profiles to a domain of economic preferences that is Arrow-inconsistent. He then shows that in order for a subset of this domain to be Arrow-consistent, it must be topologically small. Redekop has considered two different ways of formalizing the idea that a preference domain is topologically small. They both capture the idea that topologically small preference domains do not exhibit much diversity in preference. Thus, Redekop's theorems demonstrate that if the domain exhibits much preference diversity, then it is Arrow-inconsistent. More precisely, if the basic structure of the preference domain is not altered by small perturbations in the preferences, then it is Arrow-inconsistent.

In this section, we provide an introduction to Redekop's work by considering one of his theorems and its proof in some detail. The strategy used in this proof has been adapted by Redekop to show the Arrow-inconsistency of a wide variety of economic preference domains. While we briefly describe some of these results in this section, it is not possible to consider them in any detail. More detail may be found in Redekop (1995), which is a relatively nontechnical survey of the theorems established in Redekop (1991; 1993a; 1993c; 1996). We also comment on how Redekop's domain restrictions and proof strategy are related to saturating domains and the local approach.

In the theorem we consider in this section, $X=\mathbb{R}_{+}^{m}$ with $m \geq 2$. An alternative in $X$ is interpreted as being a vector of $m$ public goods. Individual

\footnotetext{
${ }^{48}$ For further discussion of Arrovian aggregation of ordinal probabiliies, see Lainé, Le Breton, and Trannoy (1986) and Weymark (1997).
} 
preferences are a priori restricted to be continuous and monotonic. Let $\mathcal{C}_{c m}$ denote the set of all such preference orderings on $X$. The universal domain of preference profiles is thus $\mathcal{C}_{c m}^{n}$.

A topology on a set $S$ is a collection of subsets $\mathcal{T}$ of $S$ that includes (i) both $S$ and $\emptyset$, (ii) all finite intersections of sets in $\mathcal{T}$, and (iii) all unions of sets in $\mathcal{T}$. The sets in $\mathcal{T}$ are the open subsets of $S$. A basis for $\mathcal{T}$ is a collection of sets contained in $\mathcal{T}$ with the property that any nonempty set in $\mathcal{T}$ is the union of sets in the basis. Thus, a topology is characterized by a basis. The sets in a basis are called basic open sets. For a topology on $\mathcal{C}_{c m}$, we use the questionnaire topology $\mathcal{T}_{Q}$ introduced in Redekop (1993b). ${ }^{49} \mathrm{~A}$ basis for $\mathcal{T}_{Q}$ is the collection of sets of the form:

$$
Q\left(\left(x_{1}, y_{1}\right), \ldots,\left(x_{r}, y_{r}\right)\right)=\bigcap_{i=1}^{r}\left\{R \in \mathcal{C}_{c m} \mid x_{i} P y_{i}\right\},
$$

where the $x_{i}$ and $y_{i}$ are alternatives in $X$. A basic open set is constructed by specifying strict rankings for a finite number of paired comparisons and then identifying all of the continuous monotonic preferences that exhibit these rankings. A subset of a topological space is somewhere dense if its closure contains an open set; otherwise, it is nowhere dense. It is natural to regard an individual preference domain as being topologically small if it is nowhere dense with respect to the questionnaire topology.

Suppose that the preference domain is common and that the individual preference domain $\mathcal{D}_{*}$ is a subset of $\mathcal{C}_{c m}$. Redekop (1991) has shown that a necessary condition for $\mathcal{D}=\mathcal{D}_{*}^{n}$ to be Arrow-consistent is that $\mathcal{D}_{*}$ be nowhere dense. Equivalently, a sufficient condition for $\mathcal{D}_{*}^{n}$ to be Arrow-inconsistent is that $\mathcal{D}_{*}$ be somewhere dense.

Theorem 7. If $X=\mathbb{R}_{+}^{m}$ with $m \geq 2$ and $\mathcal{D}_{*} \subseteq \mathcal{C}_{c m}$ is somewhere dense, then there is no social welfare function $F: \mathcal{D}_{*}^{n} \rightarrow \mathcal{R}$ that satisfies IIA, WP, and ND.

Thus, a preference domain $\mathcal{D}_{*}^{n} \subseteq \mathcal{C}_{c m}^{n}$ is Arrow-inconsistent if the closure of the individual preference domain contains at least one basic open set of preferences. A basic open set of preferences exhibits sufficient preference diversity for Arrow's axioms to be incompatible because, in addition to the

\footnotetext{
${ }^{49}$ More familiar topologies for a space of preferences are the Kannai (1970) topology and the topology of closed convergence. Redekop (1993b) has shown that these two topologies coincide with the questionnaire topology on $\mathcal{C}_{c m}$.
} 
restrictions imposed by continuity and monotonicity, the pairwise rankings are only restricted on a finite number of pairs of alternatives.

Theorem 7 is established by showing that there is a dictator if the individual preference domain is somewhere dense and the social welfare function $F$ satisfies IIA and WP. The proof has three main parts. First, it is shown that for every $x \in X$, there is an open set $O(x) \subseteq X$ containing $x$ and an individual $d(x) \in N$ such that $d(x)$ is a dictator on $O(x)$. Such an individual is called a local dictator. Second, it is shown that the same individual, say $d$, is a local dictator for all $x \in X$. These results only establish that $d$ is a dictator on pairs of alternatives that are close together in the sense that they are in the same open set $O(x)$ for some $x \in X$. The final part of the proof shows that, in fact, $d$ is a dictator on all of $X$.

To show that there is a local dictator for each $x \in X$, Redekop used an innovative version of the local approach. To describe it, we need to introduce some further definitions. A subset $A$ of $X$ is strictly free with respect to $\mathcal{D}_{*}$ if $\left.\left.\mathcal{L}\right|_{A} \subseteq \mathcal{D}_{*}\right|_{A}$; i.e., every linear ordering of the alternatives in $A$ is feasible. Two pairs $A, B \subseteq X$ are strictly connected with respect to $\mathcal{D}_{*}$ if there exists a sequence of pairs in $X, A_{1}, \ldots, A_{r}$, with $A_{1}=A$ and $A_{r}=B$ such that $A_{j} \cup A_{j+1}$ is a strict free triple with respect to $\mathcal{D}_{*}$ for all $j=1, \ldots, r-1$. An individual $d \in N$ is a strict dictator on $\{x, y\} \subseteq X$ if $x P y$ for all $\mathbf{R} \in \mathcal{D}$ for which $x P_{d} y$ and $\neg\left(x I_{i} y\right)$ for all $i \neq d$. A set $A \subseteq X$ is coordinatewise undominated if $x \geq y$ and $y \geq x$ for all distinct $x, y \in A$.

Redekop's proof begins by identifying an open set $O^{\prime}(x)$ containing $x$ in which every triple that is coordinatewise undominated is a strict free triple. By the linear preference version of Arrow's theorem, there is a strict dictator on these triples. Next, it is shown that there is an open subset $O(x)$ of $O^{\prime}(x)$ containing $x$ in which coordinatewise undominated pairs are strictly connected, from which it follows that there is a strict dictator on all such pairs. To complete the first part of the proof, it remains to show that this implies that there is a dictator on $O(x)$. As Redekop (1991, p. 408) has noted, his approach is doubly local because he uses a version of the KalaiMuller-Satterthwaite local approach on a small open set. We now turn to the details of this argument.

Lemma 1. If $X=\mathbb{R}_{+}^{m}$ with $m \geq 2, \mathcal{D}_{*} \subseteq \mathcal{C}_{c m}$ is somewhere dense, and $F: \mathcal{D}_{*}^{n} \rightarrow \mathcal{R}$ is a social welfare function that satisfies IIA and $\mathrm{WP}$, then, for all $x \in X$, there is a local dictator.

Proof. The proof proceeds in a series of steps. The first five steps only assume 
that $\mathcal{D}_{*}$ is a somewhere dense subset of $\mathcal{C}_{c m}$.

Step 1. Let $O$ be an open subset of $\mathcal{C}_{c m}$ and $U_{R}$ be a continuous utility representation of $R \in O$. We first show that there exists an $\bar{\varepsilon}>0$ such that whenever $0<\varepsilon<\bar{\varepsilon}$ and $g: X \rightarrow[0,1]$ is a continuous nondecreasing function, then $R_{\varepsilon} \in O$, where $R_{\varepsilon}$ is defined by

$$
x R_{\varepsilon} y \leftrightarrow U_{R}(x)+\varepsilon g(x) \geq U_{R}(y)+\varepsilon g(y)
$$

for all $x, y \in X .^{50}$ Because $O$ is open, it contains a basic open set $B=$ $Q\left(\left(x_{1}, y_{1}\right), \ldots,\left(x_{r}, y_{r}\right)\right)$, where $x_{j} P y_{j}$ for all $j=1, \ldots, r$. Let

$$
\bar{\varepsilon}=\frac{1}{2} \min _{1 \leq j \leq r}\left[U_{R}\left(x_{j}\right)-U_{R}\left(y_{j}\right)\right] .
$$

It is straightforward to verify that $U_{R}\left(x_{j}\right)+\varepsilon g\left(x_{j}\right)>U_{R}\left(y_{j}\right)+\varepsilon g\left(y_{j}\right)$ for all $j=1, \ldots, r$ when $0<\varepsilon<\bar{\varepsilon}$. Hence, $R_{\varepsilon} \in B \subseteq O$.

Step 2. Consider any coordinatewise undominated subset $\{a, b, c\}$ of $X$. For all $y \in\{a, b, c\}$, let $A_{y}=\{z \in X \mid z \geq y\}$. Define the function $\rho_{y}: X \rightarrow \mathbb{R}$ by setting

$$
\rho_{y}(x)=\min _{z \in A_{y}}\|x-z\|
$$

for all $x \in X$. Let

$$
m=\min _{x \neq y} \rho_{y}(x),
$$

where the minimum is taken over pairs $\{x, y\} \subseteq\{a, b, c\}$ for which $x \neq y$. Because $x \notin A_{y}$ for all such pairs and the $A_{y}$ are closed, $m>0$. Now define the functions $h_{a}: X \rightarrow[0,1]$ and $h_{b}: X \rightarrow[0,1]$ by setting, for all $x \in X$,

$$
h_{a}(x)=1-\frac{\min \left(m, \rho_{a}(x)\right)}{m}
$$

and

$$
h_{b}(x)=1-\frac{\min \left(m, \rho_{b}(x)\right)}{m} .
$$

\footnotetext{
${ }^{50} \mathrm{~A}$ function $g: \mathbb{R}_{+}^{m} \rightarrow \mathbb{R}$ is nondecreasing if $g(x) \geq g(y)$ for all $x, y \in \mathbb{R}_{+}^{m}$ for which $x \gg y$. Note that because the inequality in this definition is weak, $g$ may not represent a monotone preference. However, because $R$ is monotone, so is $R_{\varepsilon}$ when $g$ is nondecreasing.
} 
The function $h_{\alpha}, \alpha=a, b$, assigns a value of 1 to all $x \in A_{\alpha}$, a value of 0 to all $x$ that are at least distance $m$ from $A_{\alpha}$, and a value of $1-\left\|z-A_{\alpha}\right\|$ to all other $x$. Hence, $h_{a}$ and $h_{b}$ are both continuous and nondecreasing. Further, because of the way $m$ has been chosen, $h_{a}(a)=h_{b}(b)=1$ and $h_{a}(b)=h_{a}(c)=h_{b}(a)=h_{b}(c)=0$.

Step 3. We now show that for all $x \in X$, there exists an open subset $O^{\prime}(x)$ of $X$ containing $x$ such that any coordinatewise undominated triple in $O^{\prime}(x)$ is a strict free triple with respect to the closure $\overline{\mathcal{D}}_{*}$ of $\mathcal{D}_{*}$.

Because $\mathcal{D}_{*}$ is somewhere dense in $\mathcal{C}_{c m}$, there exists a basic open set $B$ contained in $\overline{\mathcal{D}}_{*}$. Consider any $R \in B$. Let $U$ be a continuous utility representation of $R$. Define $\bar{\varepsilon}$ as in Step 1 . For $0<\varepsilon<\bar{\varepsilon}$, by the continuity of $U$ at $x$, we can choose $\delta$ sufficiently small so that

$$
\sup _{\|z-x\|<\delta}|U(x)-U(z)|<\frac{\varepsilon}{9} .
$$

Let

$$
O^{\prime}(x)=\{z \in X \mid\|z-x\|<\delta\} .
$$

To complete the proof of this step, we consider any coordinatewise undominated subset $\{a, b, c\}$ of $O^{\prime}(x)$ and, without loss of generality, show that we can find an $R^{\prime} \in B$ such that $a P^{\prime} b P^{\prime} c$.

For the set $\{a, b, c\}$, define the functions $h_{a}$ and $h_{b}$ as in Step 2. Let $g: X \rightarrow[0,1]$ be the function defined by setting, for all $z \in X$,

$$
g(z)=\frac{2}{3} h_{a}(z)+\frac{1}{3} h_{b}(z) .
$$

Because $h_{a}$ and $h_{b}$ are continuous and nondecreasing, so is $g$. Hence, using $g$ to define $R_{\varepsilon}$ as in Step 1 , we conclude that $R_{\varepsilon} \in B$ when $0<\varepsilon<\bar{\varepsilon}$.

By construction, $g(a)=\frac{2}{3}, g(b)=\frac{1}{3}$, and $g(c)=0$. Therefore,

$$
\begin{aligned}
{[U(a)+\varepsilon g(a)]-[U(b)+\varepsilon g(b)] } & =\frac{1}{3} \varepsilon+[U(a)-U(b)] \\
& \geq \frac{1}{3} \varepsilon-|U(a)-U(b)| \\
& \left.\geq \frac{1}{3} \varepsilon-|U(a)-U(x)|-\mid U(b)-U(x)\right] \\
& >\frac{\varepsilon}{9},
\end{aligned}
$$


where the last inequality follows from the choice of the $\delta$ used to construct $O^{\prime}(x)$. Hence, $a P_{\varepsilon} b$. We can similarly show that $b P_{\varepsilon} c$. Setting $R^{\prime}=R_{\varepsilon}$ for any $0<\varepsilon<\bar{\varepsilon}$ completes the proof of this step.

Step 4. Suppose that $R \in \overline{\mathcal{D}}_{*}$ is such that $\bar{x} P \bar{y} P \bar{z}$. Because sets of the form $\left\{\bar{R} \in \mathcal{C}_{c m} \mid a \bar{P} b\right\}$ are open, there must exist an $R^{\prime} \in \mathcal{D}_{*}$ such that $\bar{x} P^{\prime} \bar{y} P^{\prime} \bar{z}$. Hence, if $\{x, y, z\}$ is a strict free triple with respect to $\overline{\mathcal{D}}_{*}$, it is also a strict free triple with respect to $\mathcal{D}_{*}$. By Step 3, we therefore conclude that any coordinatewise undominated triple in $O^{\prime}(x)$ is a strict free triple with respect to $\mathcal{D}_{*}$.

Step 5. Next, we show that there exists an open set $O(x) \subseteq O^{\prime}(x)$ containing $x$ with the property that if $\{v, w, y, z\} \subset O(x)$ and both $\{v, w\}$ and $\{y, z\}$ are coordinatewise undominated, then $\{v, w\}$ and $\{y, z\}$ are strictly connected with respect to $\mathcal{D}_{*}$.

We first consider the case in which $x$ has at least two positive components. Without loss of generality, we assume that $x_{1}>0$ and $x_{2}>0$. The proof is similar to the one used to establish Step 3 in Example 8 (and illustrated in Figure 3). However, in order to use Step 4 to identify strict free triples, now the two alternatives, say $s$ and $t$, that are used to form the intermediate pairs in the connection argument must be chosen from $O^{\prime}(x)$. Further, in order for $s$ and $t$ to form a strict free triple with either $\{v, w\}$ or $\{y, z\}$, $v, w, y$, and $z$ must be sufficiently close together. This will be the case if $\{v, w, y, z\} \subset O(x)$, where

$$
O(x)=\{z \in X \mid\|z-x\|<\Delta\}
$$

for $\Delta>0$ sufficiently small that (i) $\|z-x\|<3 \Delta$ implies $z \in O^{\prime}(x)$ and (ii) $\Delta<\min \left(x_{1} / 3, x_{2} / 3\right)$. If we now let $s=x+2 \Delta(1,-1,0, \ldots, 0)$ and $t=x+2 \Delta(-1,1,0, \ldots, 0)$, we have $\{s, t\} \subset O^{\prime}(x) \backslash O(x)$. In addition, $s$ and $t$ either singly or jointly form a coordinatewise undominated set when combined with any coordinatewise undominated subset of $O(x)$. Suppose that $\{v, w\}$ and $\{y, z\}$ are coordinatewise undominated subsets of $O(x)$. It then follows from Step 4 that $\{v, w, s\},\{w, s, t\},\{s, t, y\}$, and $\{t, y, z\}$ are strict free triples. Hence, $\{v, w\}$ and $\{y, z\}$ are strictly connected with respect to $\mathcal{D}_{*}$.

If $x$ has at most one positive component, the connection argument is somewhat more complex. See Redekop (1991, pp. 416-417) for the details.

Step 6. Henceforth, we assume that $F: \mathcal{D}_{*}^{n} \rightarrow \mathcal{R}$ is a social welfare function that satisfies IIA and WP. We now show that some individual is a strict dictator for all distinct $\{y, z\} \subset O(x)$. 
If $\{y, z\}$ is coordinatewise undominated, we can find a third alternative $w \in O(x)$ such that $\{w, y, z\}$ is coordinatewise undominated. By Step 4, this is a strict free triple. Hence, by the linear preference version of Arrow's Theorem, there is a strict dictator, say $d(x)$, on this triple. By Step $5,\{y, z\}$ can be strictly connected to any other coordinatewise undominated pair in $O(x)$. Hence, by the argument used in the proof of Theorem $5, d(x)$ is a strict dictator on all coordinatewise undominated pairs in $O(x)$.

Now consider the case in which $y>z, y P_{d(x)} z$, and $\neg\left(y I_{j} z\right)$ for all $j \neq$ $d(x)$. Because preferences are monotone, this is only possible if $y P_{i} z$ for all $i \in N$. Hence, by WP, we have $y P z$. Thus, $d(x)$ is a strict dictator for all distinct $\{y, z\} \subset O(x)$.

Step 7. Finally, we show that $d(x)$ is a dictator on $O(x)$. Consider any distinct $y, z \in O(x)$ and suppose that $y P_{d(x)} z$. Let $J_{1}=\left\{j \in N \mid y P_{j} z\right\}$ and $J_{2}=\left\{j \in N \mid z P_{j} y\right\}$. Because preferences are continuous, (i) for all $j \in J_{1}$, we can find an open set $O_{j}(z)$ containing $z$ such that $y P_{j} w$ for all $w \in O_{j}(z)$ and (ii) for all $j \in J_{2}$, we can find an open set $O_{j}(z)$ containing $z$ such that $w P_{j} z$ for all $w \in O_{j}(z)$. Because $\tilde{O}=O(x) \cap_{j \in\left(J_{1} \cup J_{2}\right)} O_{j}(z)$ is an open set containing $z$, we can find a $v \in \tilde{O}$ with $v \gg z$. Everybody has a strict preference on $\{v, y\}$. In particular, $y P_{d(x)} v$. Hence, by Step 6 , we have $y P v$. WP and the monotonicity of preferences imply that $v P z$. Transitivity of $P$ then implies that $y P z$, so $d(x)$ is a dictator on $O(x)$.

The use of the linear preference version of Arrow's Theorem in the proof of Lemma 1 is essential. To see why, suppose that $\mathcal{D}_{*}=\left\{R \in \mathcal{C}_{c m} \mid \neg(x I y)\right\}$ for some coordinatewise undominated pair of alternatives $\{x, y\}$. It is clear from the definition of the questionnaire topology that $\mathcal{D}_{*}$ is itself an open set, so $\mathcal{D}_{*}$ is somewhere dense. However, $\{x, y\}$ is not part of any free triple.

The next part of the proof of Theorem 7 establishes that the same person is the local dictator for all $x \in X$.

Lemma 2. If $X=\mathbb{R}_{+}^{m}$ with $m \geq 2, \mathcal{D}_{*} \subseteq \mathcal{C}_{c m}$ is somewhere dense, and for all $x \in X$, there is a local dictator $d(x)$, then there is a $d \in N$ such that $d=d(x)$ for all $x \in X$.

Proof. For all $x \in X$, let $O(x)$ be an open set containing $x$ on which $d(x)$ is a local dictator. By Step 4 of the preceding proof, we can choose $O(x)$ sufficiently small so that every coordinatewise undominated pair in $O(x)$ is strictly free. For all $i \in N$, let $X_{i}=\left\{\cup_{x \in X} O(x) \mid i=d(x)\right\}$. These are open sets that cover $X$. If $X_{i}=\emptyset$ for all $i \neq d$, then $d$ is a local dictator for all 
$x \in X$. Because $X$ is connected, the only other possibility is that there exist distinct $i, j \in N$ such that $X_{i} \cap X_{j}$ contains a coordinatewise undominated pair $\{y, z\}$. But then there exists a profile $\mathbf{R} \in \mathcal{D}$ in which $y P_{i} z$ and $z P_{j} y$. Because both $i$ and $j$ dictate on this pair, we have a contradiction. Hence, this case is not possible.

The proof of Theorem 7 is completed by showing that the local dictator in Lemma 2 in fact dictates on all of $X$. The proof makes use of a property of preferences that Redekop (1991) calls the continuous climb property. A preference ordering $R$ on $X$ (i) satisfies the continuous climb property for the ordered pair $(x, y)$ if $x P y$ and there exists a continuous function $\gamma:[0,1] \rightarrow X$ such that $\gamma(0)=y, \gamma(1)=x$, and $\gamma(s) P \gamma(t)$ whenever $s>t$ and (ii) it satisfies the continuous climb property if it satisfies the continuous climb property for all ordered pairs $(x, y)$ for which $x P y$. It is quite easy to show that if $R$ is strictly monotonic, then $R$ has the continuous climb property. Unfortunately, if a preference is monotonic, but not strictly monotonic, such a path may not exist if $x$ or $y$ is on the boundary of $X$. The following example, due to Redekop (1991), illustrates the problem.

Example 16. Let $X=\mathbb{R}_{+}^{2}$ and suppose that $R$ is represented by a utility function $U$ for which (i) $U(x)=x_{1}$ if $x_{1} \leq 1$ and (ii) $U(x)=1+\left(x_{1}-1\right) x_{2}$ otherwise. Note that $R \in \mathcal{C}_{c m}$. Let $x=(2,0)$ and $y=(0,1)$. We have $x P y$, but there is no alternative near $x$ that is worse than $x$ according to $R$ and, hence, no path with the continuous climb property connects $x$ and $y$.

Proof of Theorem \%. Let $d$ be the local dictator identified in Lemma 2. Consider a profile $\mathbf{R} \in \mathcal{D}$ and a pair of alternatives $x, y \in X$ for which $x P_{d} y$.

First, suppose that $R_{d}$ satisfies the continuous climb property for $(x, y)$ and let $\Gamma$ be a continuous monotonic path (with respect to $R_{d}$ ) from $y$ to $x$. For all $z \in \Gamma$, let $O(z)$ be the open set identified in Lemma 1 . Because these sets cover $\Gamma$ and $\Gamma$ is compact, a finite subset of these sets also covers $\Gamma$. Let $\left\{O\left(z_{1}\right), \ldots, O\left(z_{r}\right)\right\}$ be such a finite cover, where $z_{r} P_{d} z_{r-1} P_{d} \cdots P_{d} z_{1}$. Let $\left\{x_{1}, \ldots, x_{r+1}\right\}$ be such that $\left\{x_{k}, x_{k+1}\right\} \subset O\left(z_{k}\right)$ for all $k=1, \ldots, r$ with $x_{1}=y$ and $x_{r}=x$. Because $d$ dictates on each of the $O\left(z_{k}\right)$, we have $x_{k+1} P x_{k}$ for all $k=1, \ldots, r$. Transitivity then implies that $x P y$.

Now, suppose that $R_{d}$ does not satisfy the continuous climb property for $(x, y)$. This can only occur if $x$ is on the boundary of $X$ with $x \neq 0_{m}$. If $y=\lambda x$ for some $\lambda \in[0,1)$, then for any strictly monotonic preference $R_{d}^{\prime} \in \mathcal{D}_{*}$, the profiles $\mathbf{R}$ and $\mathbf{R}^{\prime}=\left(R_{1}, \ldots, R_{d-1}, R_{d}^{\prime}, R_{d+1}, \ldots, R_{n}\right)$ coincide 
on $\{x, y\}$. Because $R_{d}^{\prime}$ satisfies the continuous climb property, by the first part of this proof, we have $x P^{\prime} y$. Hence, by IIA, we also have $x P y$.

The argument when $y \neq \lambda x$ for some $\lambda \in[0,1)$ is quite lengthy, so we only sketch the main idea. A complete proof may be found in Redekop (1991). In this case, it can be shown that there exist $\bar{\lambda} \in(0,1), z \in \mathbb{R}_{++}^{m}$, and $R_{d} \in \mathcal{C}_{c m}$ such that (i) $d$ dictates on $(\bar{\lambda} x, z)$ and (ii) $x P_{d}(\bar{\lambda} x) P_{d} z P_{d} y$. We have already established that $x P(\bar{\lambda} x)$ and $z P y$. Because $d$ dictates on $(\bar{\lambda} x, z)$, we therefore have $x P y$ by the transitivity of $R$.

The complications that arise in the last step of the proof would not occur if the domain only included strictly monotonic preferences or if $X=\mathbb{R}_{++}^{m}$. With either of these two assumptions, only minor modifications need to be made to the preceding proof to show that a somewhere dense set of profiles $\mathcal{D} \subseteq \mathcal{C}_{c m}^{n}$ is Arrow-inconsistent, even if $\mathcal{D}$ is not the Cartesian product of individual preference domains. ${ }^{51}$

The profiles that create problems in the proof of Theorem 7 have the property that individual $d$ has a preference $R_{d}$ that (i) is monotonic, but not strictly monotonic, and (ii) is not contained in the closure of any open set in $\mathcal{D}_{*}$. Redekop's alternative definition of a topologically small set rules out this possibility by requiring that when any profile $\mathbf{R}$ is feasible, so is some open set containing $\mathbf{R}$, as would be the case if the true profile cannot be identified with precision. Formally, a domain of preferences $\mathcal{D}_{*}$ (resp. preference profiles $\mathcal{D}$ ) is near-open if $\overline{\mathcal{D}}_{*}=\bar{O}$ for some open set $O$ of preferences (resp. if $\overline{\mathcal{D}}=\bar{O}$ for some open set $O$ of preference profiles). Clearly, if $\mathcal{D}_{*}$ is near-open, then $\mathcal{D}_{*}$ is somewhere dense in $\mathcal{C}_{c m}$. However, the reverse implication need not hold. For example, if $\mathcal{D}_{*}=\left\{R \in \mathcal{C}_{c m} \mid x P y\right\} \cup \bar{R}$, where $\{x, y\}$ is coordinatewise undominated, $\bar{R} \in \mathcal{C}_{c m}$, and $y \bar{P} x$, then $\mathcal{D}_{*}$ is somewhere dense, but not nearopen. Without requiring that $\mathcal{D} \subseteq \mathcal{C}_{c m}^{n}$ be a Cartesian product, Redekop (1991) has shown that near-openness of $\mathcal{D}$ is sufficient for $\mathcal{D}$ to be Arrowinconsistent when $X=\mathbb{R}_{+}^{n}$.

In view of Redekop's impossibility theorems for somewhere dense and near-open domains and the impossibility results for saturating domains presented in Sections 4 and 5, it is natural to enquire if there is any logical relationship between these domain restrictions. Using the definition of the questionnaire topology, it is easy to verify that the domain of continuous, strictly monotonic, convex preferences $\mathcal{C}_{p u}$ in Example 8 is nowhere dense, although

\footnotetext{
${ }^{51}$ The product topology is used for the domain of preference profiles $\mathcal{C}_{c m}^{n}$. A basis for this topology is $\mathcal{T}_{Q}^{n}$, the $n$-fold Cartesian product of $\mathcal{T}_{Q}$.
} 
we know that it is saturating. Suppose that $\{x, y\}$ is coordinatewise undominated and $\bar{R} \in \mathcal{C}_{c m}$ is such that $x \bar{I} y$. Let $\mathcal{D}=\left[\left\{R \in \mathcal{C}_{c m} \mid x P y \text { or } y P x\right\} \cup \bar{R}\right]^{n}$. Because $\{x, y\}$ is a free pair that is not part of any free triple, this domain is not saturating. However, $\overline{\mathcal{D}}=\mathcal{C}_{c m}^{n}$, so the domain is near-open and, hence, somewhere dense. Thus, the concept of a saturating domain and Redekop's domain restrictions are logically independent. ${ }^{52}$

Redekop (1991) has noted that there is a sense in which somewhere dense domains are unreasonably large. For the public goods problem considered in this section, we have seen that this domain condition ensures that there is sufficient preference diversity so that each alternative is contained in some open set whose coordinatewise undominated triples are strictly free. However, because a basic open set $B$ is constructed by specifying strict rankings for only a finite number of paired comparisons and then identifying all of the continuous monotonic preferences that exhibit these rankings, no restrictions other than continuity and monotonicity are placed on preferences in $B$ if we remove a neighbourhood of the origin from $X$ that is large enough to contain all the alternatives that are used to generate $B$. Hence, requiring a domain to be somewhere dense also implies that any basic open set of preferences coincides with $\mathcal{C}_{c m}$ on sets of alternatives that are sufficiently far from the origin. Thus, it could be argued, open sets of preferences permit too much preference diversity when comparing alternatives far from the origin. Redekop (1991) has identified a finer topology on the space of preferences that restricts the preference diversity on such sets without limiting the diversity that is present in the neighbourhood of each alternative and has shown that his public good impossibility theorems remain valid when this topology is used to define open sets of preferences.

In Theorem 7, preferences have been a priori restricted to be continuous and monotonic. As we have seen, the domain of continuous, strictly monotonic, convex preferences $\mathcal{C}_{p u}$ in Example 8 is nowhere dense relative to $\mathcal{C}_{c m}$, so Theorem 7 does not apply to the domain of classical public goods preferences. If we are confident that everyone's preferences lie in $\mathcal{C}_{p u}$, this is the "universal" set of preferences that should be used when identifying a domain of preferences as being topologically small. Similarly, if there are other restrictions on preferences that we are confident are satisfied, then the universal

\footnotetext{
${ }^{52} \mathrm{~A}$ domain is strictly saturating if there are at least two strictly free pairs and every pair of strictly free pairs is strictly connected. Redekop (1991) has shown that if $X=\mathbb{R}_{++}^{m}$, $\mathcal{D}$ is a common preference domain, and $\mathcal{D}$ is a near-open subset of $\mathcal{C}_{c m}^{n}$, then $\mathcal{D}$ is strictly saturating.
} 
set of preferences should be restricted even further. Remarkably, Redekop has shown that his public goods impossibility theorems are quite robust to the specification of the underlying class of preferences. For example, Redekop (1993a) has shown that $\mathcal{D}_{*}^{n}$ is Arrow-inconsistent if $\mathcal{D}_{*}$ is a near-open subset of $\mathcal{C}_{p u}$ or if it is a near-open subset of the homothetic preferences in $\mathcal{C}_{p u}{ }^{53}$ Similarly, we may know that preferences lie in some parametric class, say the set of Cobb-Douglas preferences, the set of linear preferences considered in Example 9, or the set of Euclidean spatial preferences considered in Example 12. Provided that this parametric class is sufficiently rich, as in these three examples, Redekop (1993c) has shown that the incompatibility of Arrow's axioms can only be avoided on topologically small subsets of these domains when there are at least three goods. ${ }^{54}$

In addition to the public goods economies discussed here, Redekop (1993a; 1996) has considered private goods economies (see Section 9), economies with both public and private goods, economies with stochastic alternatives, deterministic intertemporal economies with an infinite number of time periods, and infinite-horizon economies with uncertain consumption streams. In each case, the basic message conveyed by Theorem 7 is unchanged-Arrowconsistent domains are topologically small. ${ }^{55}$ These results are surveyed in Redekop (1995).

\section{Supersaturating preference domains}

So far, we have restricted attention to problems in which the social alternatives are purely public. As a consequence, the analysis is not immediately applicable to problems with private goods. As we shall see, the techniques used to study public alternatives in Sections 4 and 5 can be readily adapted to study private alternatives as well. However, to fully exploit the structure imposed on the problem by the private goods assumption, it is necessary to consider refinements of the concept of a saturating preference domain. In this section, we consider a restriction on the individual preference domains that

\footnotetext{
${ }^{53}$ It is an open question if these results can be extended to preference domains that are not common.

${ }^{54}$ The restriction that $m \geq 3$ is essential in some cases, as we know from our discussion of the set of linear preferences in Example 9.

${ }^{55}$ When there are private goods, Redekop deletes the origin from each person's consumption set. As we shall see in the next two sections, standard domains of economic preferences for private goods are Arrow-consistent if this is not done.
} 
ensures that the domain of preference profiles is saturating when individuals only care about their own private consumption.

For the private domains we consider in this and the following two sections, $X$ is a Cartesian set of alternatives.

Cartesian Set of Alternatives. $X=\prod_{i=1}^{n} X_{i}$.

Thus, a social alternative is a vector $\left(x^{1}, \ldots, x^{n}\right)$, where $x^{i} \in X_{i}$ is the component of the social alternative relevant to person $i$. We refer to $x^{i}$ as $i$ 's consumption bundle and to $X_{i}$ as $i$ 's consumption set. In adopting this terminology, we are not requiring consumption bundles to be vectors in a Euclidean space, although that is in fact the case in the applications we consider. We let $\left(y^{i} ; x_{-i}\right)$ denote $\left(x^{1}, \ldots, x^{i-1}, y^{i}, x^{i+1}, \ldots, x^{n}\right)$, where $x_{-i}=\left(x^{1}, \ldots, x^{i-1}, x^{i+1}, \ldots, x^{n}\right) .{ }^{56}$

As in the previous sections, we maintain the assumption that $\mathcal{D}$ is a Cartesian preference domain. When the alternatives are private, we assume that individuals are selfish; i.e., in comparing two social alternatives, each individual is only concerned with his or her own consumption. Formally, for each $i \in N$, a preference ordering $R_{i} \in \mathcal{D}_{i}$ is selfish if there exists an ordering $Q_{i}$ on $X_{i}$ such that for all $x, y \in X, x R_{i} y$ if and only if $x^{i} Q_{i} y^{i}$, where $x^{i}$ and $y^{i}$ are $i$ 's consumption bundles in the alternatives $x$ and $y$, respectively. We refer to $Q_{i}$ as $i$ 's induced private preference.

Selfish Preference Domain. For each $i \in N$, an individual preference domain $\mathcal{D}_{i}$ on a Cartesian set of alternatives $X$ is selfish if $R_{i}$ is selfish for all $R_{i} \in \mathcal{D}_{i}$. A preference domain $\mathcal{D}$ on a Cartesian set of alternatives $X$ is selfish if the individual preference domain $\mathcal{D}_{i}$ on $X$ is selfish for each $i \in N$.

For a selfish individual preference domain $\mathcal{D}_{i}$, the set of induced private orderings of $X_{i}$ corresponding to $\mathcal{D}_{i}$ is denoted by $\mathcal{Q}_{i}$ and is called $i$ 's induced private preference domain. Note that a pair of social alternatives $x$ and $y$ is nontrivial for person $i$ with respect to $\mathcal{D}_{i}$ if and only if the corresponding pair of private consumption bundles $x^{i}$ and $y^{i}$ is nontrivial with respect to $\mathcal{Q}_{i}$. Similarly, a triple of social alternatives $\{w, x, y\}$ is a free triple with respect

\footnotetext{
${ }^{56}$ The assumption that the set of alternatives has a Cartesian structure is not satisfied in all private goods problems. For example, if the set of social alternatives is the set of allocations in an Edgeworth box, the consumption bundles of the two individuals are restricted by an overall resource constraint. We consider non-Cartesian sets of alternatives in Section 10.
} 
to $\mathcal{D}_{i}$ if and only if the corresponding triple of private consumption bundles $\left\{w^{i}, x^{i}, y^{i}\right\}$ is free with respect to $\mathcal{Q}_{i}$.

A selfish preference domain (provided each person is not indifferent between all alternatives) is clearly not common. Consequently, Theorem 5, which establishes that a common saturating preference domain is Arrowinconsistent, is not relevant when individuals have selfish preferences. However, Theorem 6, which shows that there is a dictator on every nontrivial pair when the social welfare function satisfies IIA and WP and the preference domain is saturating, does apply to selfish preference domains.

In order to help identify saturating preference domains when there are private alternatives, it is useful to consider the concept of a supersaturating preference domain, which is a domain restriction introduced by Bordes and Le Breton (1989).

Supersaturating Preference Domain. An individual preference domain $\mathcal{D}_{i}$ is supersaturating if (a) $\mathcal{D}_{i}$ is saturating and (b) for all nontrivial pairs $\{x, y\}$ with respect to $\mathcal{D}_{i}$ in $X$, there exist $u, v \in X$ such that $u, v \notin\{x, y\}$ and $\{x, y, u\},\{x, y, v\},\{x, u, v\}$, and $\{y, u, v\}$ are free triples with respect to $\mathcal{D}_{i} \cdot{ }^{57}$ A preference domain $\mathcal{D}$ is supersaturating if $\mathcal{D}_{i}$ is supersaturating for all $i \in N$.

Although Bordes and Le Breton (1989) introduced the concept of a supersaturating preference domain to study selfish preferences for private alternatives, preference domains for public alternatives can be supersaturating as well. For example, the domain of classical economic preferences for public goods considered in Example 8 is a supersaturating preference domain. Recall that in this example, $X$ is $\mathbb{R}_{+}^{m}$ with $m \geq 2$ and the common individual preference domain $\mathcal{D}_{*}$ is the set of continuous, strictly monotonic, and convex preferences $\mathcal{C}_{p u}$. We have already seen that this individual preference domain is saturating, so to establish that it is supersaturating it is sufficient to check that condition (b) is satisfied. We do this for the two-good case. Suppose that $x$ and $y$ are a nontrivial pair; i.e., neither $x>y$ nor $y>x$. These alternatives are illustrated in Figure 9. We do not rule out the possibility that either $x$ or $y$ (or both) are on one of the axes. Let $w=\left(\min \left\{x_{1}, y_{1}\right\}, \min \left\{x_{2}, y_{2}\right\}\right)$. Choose $u$ so that it is in the interior of the triangle formed by the points $w, x$, and $y$, as shown in the diagram. Our discussion of Example 8 shows that $\{x, y, u\}$ is a free triple. Choose $v$ so that it lies below the line through

\footnotetext{
${ }^{57}$ Bordes and Le Breton (1989) have shown that (a) and (b) are logically independent.
} 
$u$ and $y$, but above the lines through $u$ and $x$ and through $w$ and $y$. By again appealing to our discussion of Example 8, we conclude that $\{x, y, v\}$, $\{x, u, v\}$, and $\{y, u, v\}$ are free triples with respect to $\mathcal{D}_{*}$, which completes the demonstration that $\mathcal{D}_{*}$ is supersaturating (when $m=2$ ).

To help understand condition (b) in the definition of an individual supersaturating preference domain, we introduce the concept of a self-cycle.

Self-Cycle. A sequence of pairs $A_{1}, \ldots, A_{r}$ in $X$ is a self-cycle with respect to $\mathcal{D}_{i}($ resp. $\mathcal{D})$ if $A_{1}=A_{r}$ and $A_{j}$ and $A_{j+1}$ are strongly connected with respect to $\mathcal{D}_{i}(\operatorname{resp} . \mathcal{D})$ for all $j=1, \ldots, r-1$.

A self-cycle is a sequence of pairs connecting a pair of alternatives to itself. If condition (b) in the definition of an individual supersaturating preference domain is satisfied, then for each nontrivial pair of alternatives $\{x, y\}$, we can construct a self-cycle containing three distinct pairs and we can construct a self-cycle containing four distinct pairs. The first of these self-cycles is given by the sequence $\{x, y\},\{y, u\},\{u, x\}$, and $\{x, y\}$. The second of these selfcycles is given by the sequence $\{x, y\},\{y, u\},\{u, v\},\{v, x\}$, and $\{x, y\}$. In both of these sequences, any two adjacent pairs form a free triple. For the domain of economic preferences for public goods considered in Example 8, Figure 9 illustrates these self-cycles. By combining an appropriate number of these two self-cycles, $\{x, y\}$ can be connected to itself with a self-cycle containing $r$ pairs for any positive integer $r$ except 2,3 , and 6 .

For private alternatives, because preferences are selfish, the induced private preference domain $\mathcal{Q}_{i}$ inherits many of the structural properties of the individual preference domain $\mathcal{D}_{i}$.

Lemma 3. If $X$ is a Cartesian set of alternatives and $\mathcal{D}$ is a selfish preference domain, then for each $i \in N$, the individual preference domain $\mathcal{D}_{i}$ is saturating (resp. supersaturating) on $X$ if and only if the induced private preference domain $\mathcal{Q}_{i}$ is saturating (resp. supersaturating) on $X_{i}$.

The intuition for Lemma 3 is quite simple. Because preferences are selfish, an individual's ranking of a pair of alternatives is completely determined by the individual's preference for the corresponding consumption bundles. Hence, all of the richness in the individual preference domain $\mathcal{D}_{i}$ is embodied in the induced individual preference domain $\mathcal{Q}_{i} .{ }^{58}$

\footnotetext{
${ }^{58}$ A formal proof of Lemma 3 may be found in Le Breton and Weymark (1996).
} 
Example 17 shows that the private goods counterpart to the public preference domain considered in Example 8 is supersaturating.

Example 17. In this example, we consider the classical domain of economic preferences for private goods. Before describing our example, we first need one more definition. Suppose that $X$ is both a Cartesian set of alternatives and a subset of a Euclidean space. For each $i \in N$, a selfish preference ordering $R_{i} \in \mathcal{R}$ is strictly monotonic in own consumption if the corresponding induced private preference $Q_{i}$ is strictly monotonic on $X_{i}$.

The set of alternatives is $X=\prod_{i=1}^{n} X_{i}$, where $X_{i}=\mathbb{R}_{+}^{m}$ for all $i \in N$, where $m \geq 2$. The preference domain is the domain of classical private goods preferences, which we denote by $\mathcal{C}_{p r}$.

Classical Private Goods Preference Domain. For all $i \in N, \mathcal{D}_{i}$ is the set of all selfish, continuous, strictly monotonic in own consumption, convex preference orderings on $X$.

Because the set of alternatives is Cartesian and individual preferences are selfish, by Lemma $3, \mathcal{D}_{i}$ is supersaturating on $X$ if and only if the induced private preference domain $\mathcal{Q}_{i}$ is supersaturating on $X_{i}$. The induced private preference domain $\mathcal{Q}_{i}$ is formally the same as the public preference domain considered in Example 8. As we have already seen, this domain is supersaturating, from which it follows that $\mathcal{D}_{i}$ is supersaturating as well. Because $\mathcal{D}_{i}$ is supersaturating for all $i \in N$, the preference domain $\mathcal{D}$ is supersaturating. ${ }^{59}$

By definition, an individual preference domain $\mathcal{D}_{i}$ is saturating if it is supersaturating. In general, it does not follow that a preference domain $\mathcal{D}$ is saturating if it is supersaturating. However, if a preference domain $\mathcal{D}$ is both selfish and supersaturating, then it must also be saturating.

Theorem 8. For a Cartesian set of alternatives $X$, if the preference domain $\mathcal{D}$ is both selfish and supersaturating, then it is also saturating. ${ }^{60}$

Proof. Because the individual preference domains are saturating, for each $i \in N$, we can find alternatives $w(i), x(i), y(i)$, and $z(i)$ in $X$ such that $\{x(i), y(i)\} \neq\{w(i), z(i)\}$ and such that both pairs of alternatives are nontrivial with respect to $\mathcal{D}_{i}$. Let $w=\left(w^{1}(1), \ldots, w^{n}(n)\right), x=\left(x^{1}(1), \ldots, x^{n}(n)\right)$,

\footnotetext{
${ }^{59}$ The assumption that each person consumes the same number of private goods is not essential in this example.

${ }^{60}$ This theorem is based on Lemma 2 in Bordes and Le Breton (1989).
} 
$y=\left(y^{1}(1), \ldots, y^{n}(n)\right)$, and $z=\left(z^{1}(1), \ldots, z^{n}(n)\right)$. Because $X$ is Cartesian, each of these four alternatives is in $X$. Because preferences are selfish, the pairs $\{x, y\}$ and $\{w, z\}$ are nontrivial with respect to $\mathcal{D}$. Thus, there exist at least two nontrivial pairs with respect to $\mathcal{D}$.

Now let $\{x, y\}$ and $\{w, z\}$ be any two nontrivial pairs with respect to $\mathcal{D}$. From Lemma 3, we know that for all $i \in N$, the induced private preference domains $\mathcal{Q}_{i}$ are supersaturating on $X_{i}$ and the pairs of consumption bundles $\left\{x^{i}, y^{i}\right\}$ and $\left\{w^{i}, z^{i}\right\}$ are nontrivial with respect to $\mathcal{Q}_{i}$. Because $\mathcal{Q}_{i}$ is saturating, we can connect $\left\{x^{i}, y^{i}\right\}$ and $\left\{w^{i}, z^{i}\right\}$ using a sequence $S_{i}$ of pairs of consumption bundles starting with $\left\{x^{i}, y^{i}\right\}$ and ending with $\left\{w^{i}, z^{i}\right\}$. Because $\mathcal{Q}_{i}$ is supersaturating, we can find consumption bundles $u^{i}$ and $v^{i}$ in $X_{i}$ such that $\left\{x^{i}, y^{i}, u^{i}\right\},\left\{x^{i}, y^{i}, v^{i}\right\},\left\{x^{i}, u^{i}, v^{i}\right\}$, and $\left\{y^{i}, u^{i}, v^{i}\right\}$ are free triples with respect to $\mathcal{Q}_{i}$. Furthermore, $\left\{x^{i}, y^{i}\right\}$ is connected to itself using either the sequence of pairs (I): $\left\{x^{i}, y^{i}\right\},\left\{y^{i}, u^{i}\right\},\left\{u^{i}, x^{i}\right\}$, and $\left\{x^{i}, y^{i}\right\}$ or the sequence of pairs (II): $\left\{x^{i}, y^{i}\right\},\left\{y^{i}, u^{i}\right\},\left\{u^{i}, v^{i}\right\},\left\{v^{i}, x^{i}\right\}$, and $\left\{x^{i}, y^{i}\right\}$.

Consider the sequences $S_{1}$ and $S_{2}$. Without loss of generality, we can suppose that $S_{1}$ is no longer than $S_{2}$. Suppose $S_{2}$ has $k$ more pairs than $S_{1}$. If $k$ is positive, we add self-cycles to the beginning of $S_{1}$ and $S_{2}$ until the two sequences are the same length. If $k=1$, this is accomplished by adding one type-II self-cycle to the beginning of $S_{1}$ and by adding one type-I self-cycle to the beginning of $S_{2}$. If $k=2$, we add two type-I self-cycles to the beginning of $S_{1}$ and add one type-II self-cycle to the beginning of $S_{2}$. If $k=3$, we simply add one type-I self-cycle to the beginning of $S_{1}$. If $k>3$, by first adding an appropriate number of type-I self-cycles to the beginning of $S_{1}$, the difference in the lengths of the two sequences can be made not to exceed three, and one of the preceding procedures can be used to equate the length of the sequences.

Now that the sequences for the first two individuals are the same length, we use similar operations to equalize the length of the sequences connecting $\left\{x^{i}, y^{i}\right\}$ and $\left\{w^{i}, z^{i}\right\}$ for $i=1,2,3$. Continuing in like fashion, we equalize the lengths of the connection paths joining $\left\{x^{i}, y^{i}\right\}$ and $\left\{w^{i}, z^{i}\right\}$ for all $i \in$ $N$. For each individual, we now have a sequence of consumption bundles $\left(x^{i}, y^{i}, s^{i 1}, \ldots, s^{i t}, w^{i}, z^{i}\right)$ containing $t+4$ elements, with each adjacent pair in the sequence forming the intermediate pairs used to connect $\left\{x^{i}, y^{i}\right\}$ and $\left\{w^{i}, z^{i}\right\}$ with respect to $\mathcal{Q}_{i}$. Next, we form a sequence of alternatives in $X$ by combining these individual consumption bundles. That is, we construct the sequence $x=\left(x^{1}, \ldots, x^{n}\right), y=\left(y^{1}, \ldots, y^{n}\right), s^{1}=\left(s^{11}, \ldots, s^{n 1}\right), \ldots$, $s^{t}=\left(s^{1 t}, \ldots, s^{n t}\right), w=\left(w^{1}, \ldots, w^{n}\right), z=\left(z^{1}, \ldots, z^{n}\right)$. Each adjacent pair 
in this sequence is strongly connected, which establishes that the pairs $\{x, y\}$ and $\{w, z\}$ are connected in $\mathcal{D}$. Hence, $\mathcal{D}$ is saturating.

An immediate implication of Theorems 6 and 8 is that if a private preference domain is supersaturating, then there must be a dictator on every nontrivial pair if the social welfare function satisfies IIA and WP.

Theorem 9. For a Cartesian set of alternatives $X$, if a social welfare function on a preference domain that is both selfish and supersaturating satisfies IIA and $\mathrm{WP}$, then there is an individual $d \in N$ who is a dictator on every nontrivial pair of social alternatives.

The usefulness of Theorem 9 is limited by the fact that a pair of social alternatives is nontrivial if and only if the corresponding pairs of private consumption bundles are nontrivial for each individual. For example, with the classical economic preferences for private goods considered in Example 17, the social alternatives $x$ and $y$ are a nontrivial pair if and only if for all individuals neither $x^{i}>y^{i}$ nor $y^{i}>x^{i}$. Because this domain satisfies the assumptions of Theorem 9, there is a dictator on the nontrivial pairs. However, if we want to compare a pair of social alternatives in which even a single individual's consumption bundle is the same in both alternatives or in which some individual receives more of all goods in one of the two alternatives, then Theorem 9 tells us nothing about the social ranking.

Using the essential idea underlying an example in Blau (1957), Border (1983) has shown that the domain $\mathcal{C}_{p r}$ of classical economic preferences for private goods considered in Example 17 is Arrow-consistent. In Example 18, we present the social welfare function Border used to demonstrate this result.

Example 18. In this example, the set of alternatives $X$ and the preference domain $\mathcal{D}$ are the same as in Example 17 but with $n=2 .{ }^{61}$ By construction, the set of alternatives is Cartesian and the preference domain is selfish. We have already established that the preference domain is also supersaturating. Consider the following four subsets of $X: A_{1}=\left\{x \in X \mid x^{1} \neq 0_{m}\right.$ and $x^{2} \neq$ $\left.0_{m}\right\}, A_{2}=\left\{x \in X \mid x^{1} \neq 0_{m}\right.$ and $\left.x^{2}=0_{m}\right\}, A_{3}=\left\{x \in X \mid x^{1}=0_{m}\right.$ and $x^{2} \neq$ $\left.0_{m}\right\}$, and $A_{4}=\left\{\left(0_{m}, 0_{m}\right)\right\}$. These four sets form a partition of $X$. In $A_{1}$ both individuals consume some of at least one good. In each of the other sets, at least one person receives nothing. The social welfare function is defined by setting, for all $\mathbf{R} \in \mathcal{D}$,

\footnotetext{
${ }^{61}$ It is a straightforward matter to extend this example to larger populations.
} 
(i) $\forall x, y \in A_{1}, x R y \leftrightarrow x R_{2} y$

(ii) $\forall x, y \in A_{2}, x R y \leftrightarrow x R_{1} y$,

(iii) $\forall x, y \in A_{3}, x R y \leftrightarrow x R_{2} y$,

(iv) $\forall i<j, \forall x \in A_{i}, \forall y \in A_{j}, x P y$.

This social welfare function is nondictatorial. Person two dictates on alternatives in $A_{1}$. However, person two is not an overall dictator. If $x \in A_{3}$ and $y \in A_{2}$, then $x P_{2} y$ because two's consumption is nonzero in $A_{3}$ and is zero in $A_{2}$. But, by (iv), the social preference is $y P x$.

This social welfare function satisfies WP because the social preference always agrees with the individual preference of at least one person. This fact is obvious if we compare two alternatives from the same cell in the partition. It is not difficult to verify this claim when the alternatives come from different cells. For example, if $x \in A_{3}$ and $y \in A_{2}$, the social preference coincides with person one's preference.

We leave it to the reader to confirm that IIA is satisfied and that the social preferences are orderings. ${ }^{62}$

\section{Hypersaturating preference domains}

With public alternatives, the Kalai-Muller-Satterthwaite Theorem tells us that if the preference domain is common and saturating, then it is Arrowinconsistent. In the preceding section, we learned that with a Cartesian set of private alternatives, the preference domain is saturating if the individual preference domains are selfish and supersaturating. With selfish preferences, it is not possible for individuals to have the same individual preference domains and, therefore, we cannot use the Kalai-Muller-Satterthwaite Theorem to establish that a private alternatives preference domain is Arrow-inconsistent when individuals only care about their own consumption. In this section, we consider a refinement of the concept of a supersaturating preference domain that implies that the domain is Arrow-inconsistent when the set of alternatives is Cartesian and preferences are selfish.

\footnotetext{
${ }^{62}$ Donaldson and Roemer (1987) have considered private goods environments in which the number of goods is variable. They introduced an axiom that places restrictions on how the social rankings for different numbers of goods are related to each other. When combined with Pareto Indifference (the requirement that two alternatives are socially indifferent when everyone is indifferent between them), this consistency condition implies IIA. Donaldson and Roemer have noted that their consistency axiom is violated if, for each fixed number of goods, alternatives are socially ranked as in Example 18.
} 
To help understand why a domain like the classical domain of private goods preferences considered in Examples 17 and 18 is Arrow-consistent, we introduce the concept of a trivial pair being separable for an individual.

Separable Trivial Pair. A pair of alternatives $\{x, y\}$ in $X$ that is trivial for $i$ is separable with respect to $\mathcal{D}_{i}$ if there exists a $z \in X$ such that the pairs $\{x, z\}$ and $\{y, z\}$ are nontrivial for $i$ and either (a) $x P_{i} y$ for all $R_{i} \in \mathcal{D}_{i}$ and there exists an $R_{i}^{\prime} \in \mathcal{D}_{i}$ such that $x P_{i}^{\prime} z P_{i}^{\prime} y$ or (b) $y P_{i} x$ for all $R_{i} \in \mathcal{D}_{i}$ and there exists an $R_{i}^{\prime} \in \mathcal{D}_{i}$ such that $y P_{i}^{\prime} z P_{i}^{\prime} x$.

The concept of a separable trivial pair is implicit in Bordes and Le Breton (1989). ${ }^{63}$ Informally, a trivial pair $\{x, y\}$ is separable for person $i$ if $i$ is not indifferent between $x$ and $y$ and there is an admissible preference ordering for $i$ and an alternative $z$ such that $z$ is intermediate in preference between $x$ and $y$ and such that $\{x, z\}$ and $\{y, z\}$ are nontrivial pairs for $i$.

Bordes and Le Breton (1989) used the notion of a separable trivial pair to define a hypersaturating preference domain.

Hypersaturating Preference Domain. An individual preference domain $\mathcal{D}_{i}$ is hypersaturating if (a) $\mathcal{D}_{i}$ is supersaturating and (b) all trivial pairs $\{x, y\}$ with respect to $\mathcal{D}_{i}$ in $X$ for which $\neg\left(x I_{i} y\right)$ for all $R_{i} \in \mathcal{D}_{i}$ are separable for $i$. A preference domain $\mathcal{D}$ is hypersaturating if $\mathcal{D}_{i}$ is hypersaturating for all $i \in N$.

With a hypersaturating preference domain, all nonindifferent trivial pairs are separable for each individual. The preference domain in Examples 17 and 18 is not hypersaturating because for each individual we can find trivial pairs consisting of two nonindifferent alternatives that are not separable. For example, if $x \gg y=\left(0_{m}, 0_{m}\right),\{x, y\}$ is a trivial pair for both individuals for the preference domain considered in Example 18. Both individuals prefer $x$ to $y$, but $x$ and $y$ cannot be separated.

Lemma 4 provides the analogue to Lemma 3 for hypersaturating individual preference domains.

Lemma 4. If $X$ is a Cartesian set of alternatives and $\mathcal{D}$ is a selfish preference domain, then for each $i \in N$, the individual preference domain $\mathcal{D}_{i}$ is hypersaturating on $X$ if and only if the induced private preference domain $\mathcal{Q}_{i}$ is hypersaturating on $X_{i}$.

\footnotetext{
${ }^{63} \mathrm{~A}$ closely related idea is used in Kalai and Ritz (1980).
} 
Although the preference domain of classical economic preferences for private goods is not hypersaturating when each consumption set is a nonnegative orthant, it is hypersaturating if either the origin is removed from each person's consumption set or if all goods must be consumed in positive amounts.

Example 19. In this example, the preference domain is the domain of classical private goods preferences considered in Example 17, but now the origin is removed from each person's consumption set so that $X_{i}=\mathbb{R}_{+}^{m} \backslash\left\{0_{m}\right\}$ for all $i \in N$, where $m \geq 2$.

By Lemma 4, to show that this preference domain is hypersaturating, it is sufficient to show that the induced private preference domain $\mathcal{Q}_{i}$ is hypersaturating on $\mathbb{R}_{+}^{m} \backslash\left\{0_{m}\right\}$ for all $i \in N$. The discussion of Example 17 shows that $\mathcal{Q}_{i}$ is supersaturating. (Deleting the origin from the consumption set does not affect this argument.) We illustrate the rest of the proof that $\mathcal{Q}_{i}$ is hypersaturating for the case in which $m=2$. On $X_{i}$, the pair $\left\{x^{i}, y^{i}\right\}$ is trivial for $i$ with $x^{i}$ preferred to $y^{i}$ if and only if $x^{i}>y^{i}$, as illustrated in Figure 10. The pair $\left\{x^{i}, y^{i}\right\}$ can be separated by any consumption bundle $z^{i}$ in the shaded regions of the diagram. Note that if $y^{i}$ is on one of the axes, then there is only one such region (whether or not $x^{i}$ is on this axis as well).

Example 20. This example is identical to Example 19 except that now $X_{i}=\mathbb{R}_{++}^{m}$ for all $i \in N$. The reasoning used to show that Example 19 is hypersaturating also shows that this preference domain is hypersaturating. ${ }^{64}$

Border (1983) has shown that the preference domain in Example 20 is Arrow-inconsistent. See also the related result in Maskin (1976). ${ }^{65}$ When there are two individuals, the universal set of alternatives in Example 19 is equal to the set $A_{1}$ in Example 18. Recall that the social welfare function in Example 18 is dictatorial on $A_{1}$. Theorem 10 shows that this is no accident. For a Cartesian set of alternatives and selfish preferences, Theorem 10 demonstrates that a preference domain is Arrow-inconsistent if it is hypersaturating. This result, which is due to Bordes and Le Breton (1989), is a

\footnotetext{
${ }^{64}$ See Bordes and Le Breton (1989) for other examples of hypersaturating preference domains with private alternatives.

${ }^{65}$ Arrow (1951, Chapter VI) showed that free triples exist when there are private goods and individuals have selfish preferences that are monotone in own consumption. The original (erroneous) version of Arrow's impossibility theorem did not utilize Unrestricted Domain, but instead simply assumed that there exists a free triple. This lead Arrow to falsely conclude that a private-goods version of his impossibility theorem follows immediately from his more general theorem.
} 
private good analogue to the Kalai-Muller-Satterthwaite Theorem (Theorem 5) for public alternatives.

Theorem 10. For a Cartesian set of alternatives $X$, if a social welfare function on a preference domain that is both selfish and hypersaturating satisfies IIA and WP, then it is dictatorial.

Proof. Because a hypersaturating preference domain is supersaturating, it follows from Theorem 9 that there is an individual $d$ who is a dictator on the nontrivial pairs. We show that $d$ is also a dictator on the trivial pairs.

Consider any trivial pair $\{x, y\}$ and let $\mathbf{R}=\left(R_{1}, \ldots, R_{n}\right)$ be any profile in $\mathcal{D}$ for which $x P_{d} y$. We now show that there is an alternative $z \in X$ such that for all $i \in N$, (i) $\left\{x^{i}, z^{i}\right\}$ and $\left\{y^{i}, z^{i}\right\}$ are nontrivial pairs of consumption bundles for the induced private preference domain $\mathcal{Q}_{i}$ and (ii) there exists an induced private preference $Q_{i}^{\prime} \in \mathcal{Q}_{i}$ for which $i$ weakly prefers $x^{i}$ to $z^{i}$ (resp. $z^{i}$ to $x^{i}$ ) and weakly prefers $z^{i}$ to $y^{i}$ (resp. $y^{i}$ to $z^{i}$ ) if and only if $i$ weakly prefers $x^{i}$ to $y^{i}$ (resp. $y^{i}$ to $x^{i}$ ) according to $Q_{i}$. Note that the pair of consumption bundles $\left\{x^{i}, y^{i}\right\}$ need not be trivial for all $i$. By Lemma 4, the induced private preference domain $\mathcal{Q}_{i}$ is hypersaturating for all $i \in N$. Because $\mathcal{Q}_{i}$ is saturating, if $\left\{x^{i}, y^{i}\right\}$ is nontrivial for $i$, there must be a free triple with respect to $\mathcal{Q}_{i}$ that includes $\left\{x^{i}, y^{i}\right\}$. In this case, $z^{i}$ can be chosen to be the third member of this triple and the requisite preference clearly exists. If $\left\{x^{i}, y^{i}\right\}$ is trivial for $i$, it follows immediately from the fact that $\mathcal{Q}_{i}$ is hypersaturating that $z^{i}$ and $Q_{i}^{\prime}$ exist when $i$ is not indifferent between $x^{i}$ and $y^{i}$ according to $Q_{i}$. If $i$ is indifferent, the argument is more involved. See Bordes and Le Breton (1989) or Le Breton and Weymark (1996) for the details. Because preferences are selfish, it follows that $\{x, z\}$ and $\{y, z\}$ are nontrivial pairs of social alternatives.

For all $i \in N$, let $R_{i}^{\prime}$ be the selfish preference on $X$ corresponding to $Q_{i}^{\prime}$ and let $\mathbf{R}^{\prime}=\left(R_{1}^{\prime}, \ldots, R_{n}^{\prime}\right)$. By construction, $x P_{d}^{\prime} z$ and $z P_{d}^{\prime} y$. Because $\{x, z\}$ and $\{y, z\}$ are nontrivial pairs, $d$ is a dictator on these two pairs. Thus, $x P^{\prime} z$ and $z P^{\prime} y$. By the transitivity of social preference, it follows that $x P^{\prime} y$. IIA then implies that $x P_{d} y$. Hence, person $d$ is a dictator. ${ }^{66}$

\footnotetext{
${ }^{66}$ The argument we used to show that $d$ is a dictator on the ordered pair $(\{1,2\},\{1\})$ in our discussion of ordinal probabilities (Example 15) is similar. As in the proof of Theorem 10 , we introduced a third pair of alternatives $\{1,3\}$ to separate $\{1,2\}$ from $\{1\}$. The pairs $\{\{1,2\},\{1,3\}\}$ and $\{\{1,3\},\{1\}\}$ are both nontrivial, but only the former is free. Because $\{\{1,3\},\{1\}\}$ is not a free pair, we had to use IIA and WP before we could appeal to
} 
The classical domain of economic preferences for private goods is Arrowconsistent if the origin is included in the individual consumption sets, as in Examples 17 and 18. On this domain, the preceding argument does not apply whenever the pair $\{x, y\}$ includes the origin, as such a pair cannot be separated. However, it follows from Theorem 10 and Example 19 that the only social welfare functions that satisfy all of the Arrow axioms on such domains are dictatorial on the subset of alternatives obtained by deleting the origin from each person's consumption set. In other words, only if at least one of the alternatives being compared has someone with zero consumption may the individual who is "almost dictatorial" have his or her strict preference overridden. The social welfare function in Example 18 has this property. It thus seems that with private alternatives, just like with public alternatives, restricting the preference domain does not provide a satisfactory way of avoiding Arrow's dilemma.

\section{Topological domain restrictions for private goods}

Redekop (1993a) has established private goods counterparts to the topological public goods results discussed in Section 6. In this section, we describe a private good analogue to Theorem 7 . We also consider a theorem of Redekop's that shows that a preference domain for private goods can be Arrowconsistent even if everyone has identical preferences for own consumption.

Throughout this section, the set of alternatives is $X=\prod_{i=1}^{n} X_{i}$, where $X_{i}=\mathbb{R}_{+}^{m} \backslash\left\{0_{m}\right\}$ for all $i \in N$ with $m \geq 2$. We also assume that preferences are selfish. In Redekop's "identical preferences" theorem, the preference domain $\mathcal{D}$ is not Cartesian. When this is the case, $\mathcal{D}$ is selfish if for all $\mathbf{R} \in \mathcal{D}$ and all $i \in N, R_{i}$ is selfish.

Let $\mathcal{C}_{c s m}$ denote the set of continuous and strictly monotonic preference orderings on $\mathbb{R}_{+}^{m} \backslash\left\{0_{m}\right\}$. Redekop (1993a) has shown that a selfish Cartesian preference domain is Arrow-inconsistent if each person has an induced individual preference domain that is a near-open subset of $\mathcal{C}_{c s m}$.

Theorem 11. If $X=\left(\mathbb{R}_{+}^{m} \backslash\left\{0_{m}\right\}\right)^{n}$ with $m \geq 2$, the Cartesian preference domain $\mathcal{D}$ is selfish, and, for all $i \in N$, the induced individual preference domain $\mathcal{Q}_{i}$ is a near-open subset of $\mathcal{C}_{c s m}$, then there is no social welfare function $F: \mathcal{D} \rightarrow \mathcal{R}$ that satisfies IIA, WP, and ND.

transitivity, and we could not use our finding that $d$ is a dictator on $(\{1,2\},\{1\})$ to also conclude that $d$ is a dictator on $(\{1\},\{1,2\})$. 
Note that the individual preference domains for own consumption in this theorem can be person specific. The strategy used to prove Theorem 11 is similar to the one used to prove Theorem 7, except for the way that the open sets are constructed on which an individual is a local dictator. See Redekop (1993a) for the details. By assuming that preferences for own consumption are strictly monotonic, not just monotonic, and by assuming that each $\mathcal{Q}_{i}$ is a near-open subset of $\mathcal{C}_{c s m}$, rather than being simply a somewhere dense subset of $\mathcal{C}_{c s m}$, the technical problems described in Section 6 can be avoided.

With public goods, if the preference domain is restricted so that everyone always has identical preferences, then no pair or triple of alternatives is strictly free. However, with private goods, even if everyone has identical preferences for own consumption, it is relatively easy to construct strict free triples, provided that the common induced individual preference domain exhibits sufficient preference diversity. The reason for this is that each individual's consumption bundle can be varied independently. For example, suppose that $\mathcal{Q}_{i}=\mathcal{C}_{c s m}$ for all $i \in N$, but in any admissible preference profile, everyone has the same induced private preference. Consider three alternatives $x, y$, and $z$ for which the $3 n$ vectors of private consumptions $x^{1}, y^{1}, z^{1}, \ldots, x^{n}, y^{n}, z^{n}$ are coordinatewise undominated. It is easy to see that $\{x, y, z\}$ is a strict free triple, even though everyone has the same preferences for own consumption.

A selfish preference domain for private goods $\mathcal{D}$ has a common private preference domain if $\mathcal{Q}_{i}=\mathcal{Q}_{j}$ for all $i, j \in N$. Let $\mathcal{Q}$ denote this common set of preferences for own consumption. A selfish preference domain for private goods $\mathcal{D}$ exhibits identity of preferences for own consumption if for all $\mathbf{R} \in \mathcal{D}, Q_{i}=Q_{j}$ for all $i, j \in N$. Redekop (1993a) has shown that a preference domain is Arrow-inconsistent if the domain has both of these properties and $\mathcal{Q}$ is a near-open subset of $\mathcal{C}_{c s m}$.

Theorem 12. If $X=\left(\mathbb{R}_{+}^{m} \backslash\left\{0_{m}\right\}\right)^{n}$ with $m \geq 2$, the preference domain $\mathcal{D}$ is selfish with a common private preference domain $\mathcal{Q}, \mathcal{D}$ exhibits identity of preferences for own consumption, and $\mathcal{Q}$ is a near-open subset of $\mathcal{C}_{c s m}$, then there is no social welfare function $F: \mathcal{D} \rightarrow \mathcal{R}$ that satisfies IIA, WP, and ND.

The proof of Theorem 12 is similar to the proof of Theorem 11. See Redekop (1993a). An implication of this theorem is that the Cartesian structure of the alternatives and the selfishness of preferences play significant roles in 
generating the preference diversity needed to show that a domain is Arrowinconsistent when there are private goods.

As in Section 6, the theorems presented in this section are quite robust to the specification of the "universal" set of preferences that is used to identify when a domain is topologically large. In particular, these theorems also hold when $\mathcal{C}_{c s m}$ is replaced by the set of continuous, strictly monotonic, and convex preference orderings on $\mathbb{R}_{+}^{m} \backslash\left\{0_{m}\right\}$. See Redekop (1993a; 1995). Thus, Redekop's topological approach can be used to provide an alternative proof that the domain of classical private goods preferences $\mathcal{C}_{p r}$ in Example 19 is Arrow-inconsistent.

\section{Non-Cartesian sets of alternatives}

So far, our discussion of private alternatives has assumed that the set of alternatives is a Cartesian product set $\prod_{i=1}^{n} X_{i}$. In this section, the set of alternatives is a proper subset of a product set. If an alternative is an allocation of goods, this setting arises naturally when the set of alternatives $X$ incorporates feasibility constraints - allocation decisions for one individual limit what can be done for the remaining individuals. There are no general results when the set of alternatives is not Cartesian. However, Bordes and Le Breton (1990a) and Bordes, Campbell, and Le Breton (1995) have shown that a modification of the local approach permits one to establish Arrowinconsistency in some important non-Cartesian examples. We consider their analyses of the assignment problem and of exchange economies. ${ }^{67}$

\subsection{Assignments of indivisible objects}

We assume that there is a set $G$ of $n$ indivisible goods. Without loss of generality, we can assume that $G=N=\{1,2, \ldots, n\}$. For example, the elements of $G$ could be seats in a theater, places to park a car, dorm rooms in a college, etc. In terms of our earlier notation, for all $i \in N$, $i$ 's consumption set $X_{i}$ is $G$. However, each object can only be assigned to one individual, so the set of feasible alternatives $X$ is a proper subset of $G^{n}$. Thus, an alternative is an assignment of the $n$ goods to the $n$ individuals, each individual receiving exactly one good. Formally, an assignment $x$ is a bijection from $N$ onto $G$.

\footnotetext{
${ }^{67}$ Non-Cartesian sets of alternatives that arise because of feasibility constraints are also a feature of the overlapping generations economies considered by Campbell (1992b; 1992d).
} 
Because $G=N$, we can identify $X$ with $\Sigma_{n}$, the set of permutations of $N$. In the subsequent discussion, we single out two kinds of permutations. A transposition transposes two elements of $N$. Let $\Theta_{n}$ denote the set of transpositions of $N$. For all $i \in N$, a circular permutation maps $i$ into $i+t$ for some $t \in\{0, \ldots, n-1\}$, where addition is modulo $n$. Let $C_{n}$ denote the set of circular permutations of $N$. For $\sigma \in \Sigma_{n}, \sigma C_{n}$ is the set obtained by applying the permutation $\sigma$ to each of the elements in $C_{n}$.

It is assumed that an individual only cares about the good allocated to him or her. In other words, for all $i \in N$, all $R_{i} \in \mathcal{D}_{i}$, and all $x, y \in X$, if $x_{i}=y_{i}$, then $x I_{i} y$. We make no further restrictions on individual preferences other than that they lie in $\mathcal{R}$ (the set of orderings of $X$ ). Note that this implies that all of the objects in $G$ must be different. Let $\mathcal{R}_{i A}$ denote $i$ 's set of admissible individual preferences.

Bordes and Le Breton (1990a) have shown that if $n \geq 4$, then the preference domain $\prod_{i=1}^{n} \mathcal{R}_{i A}$ is Arrow-inconsistent. Before considering their theorem, we first show why the $n=3$ case is not covered by their result.

Example 21. When $n=3, X$ contains six alternatives. We group them into the sets $A_{1}=\{(1,2,3),(2,3,1),(3,1,2)\}$ and $A_{2}=\{(1,3,2),(3,2,1),(2,1,3)\}$. In each of these alternatives, the $i$ th component is the good assigned to person $i$. Note that $A_{1}$ is the set of circular permutations $C_{3},(1,3,2)$ is obtained by transposing the assignment of goods 2 and 3 in $(1,2,3)$, and $A_{2}$ is the set of assignments obtained by applying circular permutations to $(1,3,2)$. Both $A_{1}$ and $A_{2}$ are free triples, so any social welfare function with preference domain $\Pi_{i=1}^{n} \mathcal{R}_{i A}$ is dictatorial on each of these triples if IIA and WP are satisfied.

Define the social choice welfare function $F: \prod_{i=1}^{n} \mathcal{R}_{i A} \rightarrow \mathcal{R}$ by setting, for all $\mathbf{R} \in \Pi_{i=1}^{n} \mathcal{R}_{i A}$,

(i) $x R y \leftrightarrow x R_{1} y$ for $x, y \in A_{1}$,

(ii) $x R y \leftrightarrow x R_{2} y$ for $x, y \in A_{2}$,

(iii) $x P y$ if $x \in A_{1}$ and $y \in A_{2}$.

For the same reason as in Example 2, this is a well-defined social welfare function. $F$ satisfies all the Arrow axioms. ${ }^{68}$

Theorem 13 is Bordes and Le Breton's impossibility theorem for the assignment problem.

\footnotetext{
${ }^{68}$ Note that if $x \in A_{1}$ and $y \in A_{2}$, the weak Pareto principle is vacuous because these alternatives are not Pareto ranked (someone receives the same good in both assignments). We also have dictators on the two subsets of alternatives in Example 2, but the two sets are Pareto ranked in that example.
} 
Theorem 13. If $X=\Sigma_{n}$ and $n \geq 4$, there is no social welfare function with preference domain $\prod_{i=1}^{n} \mathcal{R}_{i A}$ that satisfies IIA, WP, and ND.

Proof. We present the the proof for the case of $n \geq 5 .{ }^{69}$ Suppose that $F: \prod_{i=1}^{n} \mathcal{R}_{i A} \rightarrow \mathcal{R}$ satisfies IIA and WP. The proof proceeds in a series of steps.

Step 1. We first show that for all $\sigma \in \Sigma_{n}$, there is a dictator $d(\sigma)$ on $\sigma C_{n}$. Because each person receives a different object in each alternative in $C_{n}$, preferences are unrestricted on $C_{n}$. For the same reason, preferences are unrestricted on any common permutation of the alternatives in $C_{n}$. Hence, the existence of a dictator on $\sigma C_{n}$ follows from Arrow's Theorem.

Step 2. We next show that for all $\sigma \in \Sigma_{n}$, all $\theta \in \Theta_{n}$, and all $x \in C_{n}$, there exist $x^{\prime}, x^{\prime \prime} \in C_{n}$ such that $\left\{\sigma x, \theta \sigma x^{\prime}, \theta \sigma x^{\prime \prime}\right\}$ is a free triple. ${ }^{70}$ Suppose that $\theta$ transposes the assignment of goods $i$ and $j$. We have already seen that each person receives a different object in each alternative in $\sigma C_{n}$. If for all alternatives in $\sigma C_{n}$ except $\sigma x$, we transpose the assignment of goods $i$ and $j$, it will still be the case that everyone receives a different object in each alternative in $\theta \sigma C_{n} \backslash\{\theta \sigma x\}$. To complete the proof of this step, we need to show that there are at least two of these transposed alternatives, say $\theta \sigma x^{\prime}$ and $\theta \sigma x^{\prime \prime}$, in which everyone receives different objects in $\left\{\sigma x, \theta \sigma x^{\prime}, \theta \sigma x^{\prime \prime}\right\}$.

Let $i^{*}=(\sigma x)^{-1}(i)$ and $j^{*}=(\sigma x)^{-1}(j)$ be the individuals who receive objects $i$ and $j$, respectively, in $\sigma x$. For any $k \neq i^{*}, j^{*}$, transposing the assignment of objects $i$ and $j$ in $\sigma x$ has no effect on what $k$ receives, so $\sigma x(k)=\theta \sigma x(k)$. For any circular permutation $y$ distinct from $x, x(k) \neq y(k)$ and, hence, $\sigma x(k) \neq \theta \sigma y(k)$ for $k \neq i^{*}, j^{*}$. For each individual and each object, there is exactly one assignment in $C_{n}$ in which this individual receives this object. Hence, there exists exactly one $x_{i^{*}} \in C_{n}$ such that $i=\sigma x\left(i^{*}\right)=$ $\theta \sigma x_{i^{*}}\left(i^{*}\right)$ and exactly one $x_{j^{*}} \in C_{n}$ such that $j=\sigma x\left(j^{*}\right)=\theta \sigma x_{j^{*}}\left(j^{*}\right)$.

Let $E=\theta \sigma C_{n} \backslash\left\{\theta \sigma x, \theta \sigma x_{i^{*}}, \theta \sigma x_{j^{*}}\right\}$. Because $n \geq 5,|E| \geq 2$. Let $\theta \sigma x^{\prime}$ and $\theta \sigma x^{\prime \prime}$ be any two distinct elements of $E$. For each $k \neq i^{*}, j^{*}$, from the preceding discussion, we know that $k$ receives distinct objects in $\sigma x, \theta \sigma x^{\prime}$, and $\theta \sigma x^{\prime \prime}$ because $x, x^{\prime}$, and $x^{\prime \prime}$ are distinct. Because these circular permutations are distinct, individuals $i^{*}$ and $j^{*}$ receive different objects in $\theta \sigma x, \theta \sigma x^{\prime}$, and $\theta \sigma x^{\prime \prime}$. By the construction of $E$, neither $i^{*}$ nor $j^{*}$ receive objects $i$ or $j$ in either $\theta \sigma x^{\prime}$ or $\theta \sigma x^{\prime \prime}$. Hence, $i^{*}$ and $j^{*}$ also receive distinct objects in $\sigma x, \theta \sigma x^{\prime}$,

\footnotetext{
${ }^{69}$ The case $n=4$ calls for a special treatment. See Bordes and Le Breton (1990a).

${ }^{70}$ When we compose two permutations, such as $\sigma$ and $x$, we write $\sigma x$ instead of $\sigma(x)$. Note that a permutation is a mapping from $N$ to $N$, not a permutation matrix.
} 
and $\theta \sigma x^{\prime \prime}$.

Step 3. We now show that for all $\sigma \in \Sigma_{n}$ and all $\theta \in \Theta_{n}, d(\sigma)=d(\theta \sigma)$. Let $\sigma_{1}$ be any element of $\sigma C_{n}$. By Step 2, there exist $\sigma_{2}, \sigma_{3} \in \theta \sigma C_{n}$ such that $\left\{\sigma_{1}, \sigma_{2}, \sigma_{3}\right\}$ is a free triple. As in Step 2, suppose that $\theta$ transposes the assignment of goods $i$ and $j$. Let $i^{* *}$ and $j^{* *}$ be the individuals who receive objects $i$ and $j$, respectively, in $\sigma_{2}$. Because $\theta \theta$ is the identity transform, $\theta \sigma_{2} \in \sigma C_{n}$. We know that everyone receives a different object in every assignment in $\sigma C_{n}$. Thus, for any $\sigma_{4} \in \sigma C_{n} \backslash\left\{\sigma_{1}, \theta \sigma_{2}\right\}$, everyone except possibly $i^{* *}$ or $j^{* *}$ must receive different objects in $\sigma_{1}, \sigma_{2}$, and $\sigma_{4}$. Provided that we choose $\sigma_{4}$ so that $i^{* *}$ does not get object $i$ and $j^{* *}$ does not get object $j, i^{* *}$ and $j^{* *}$ also receive different objects in $\sigma_{1}, \sigma_{2}$, and $\sigma_{4}$. This is possible because $\left|\sigma C_{n} \backslash\left\{\sigma_{1}, \theta \sigma_{2}\right\}\right| \geq 3$. Therefore, $\left\{\sigma_{1}, \sigma_{2}, \sigma_{4}\right\}$ is a free triple. The pairs $\left\{\sigma_{4}, \sigma_{1}\right\}$ and $\left\{\sigma_{2}, \sigma_{3}\right\}$ are connected using the intermediate pair $\left\{\sigma_{1}, \sigma_{2}\right\}$. Hence, the dictator $d(\sigma)$ on $\left\{\sigma_{4}, \sigma_{1}\right\}$ must be the same as the dictator on $\left\{\sigma_{2}, \sigma_{3}\right\}$.

Step 4. Next we show that there exists a $d \in N$ such that $d(\sigma)=d$ for all $\sigma \in \Sigma_{n}$. Consider any $\sigma \in \Sigma_{n}$. Any permutation can be written as the composition of transpositions, so there exist $\theta_{1}, \theta_{2}, \ldots, \theta_{q} \in \Theta_{n}$ such that $\sigma=\theta_{1} \theta_{2} \cdots \theta_{q}$. By Step 3, $d\left(\theta_{q}\right)=d\left(\theta_{q-1} \theta_{q}\right), d\left(\theta_{q-1} \theta_{q}\right)=d\left(\theta_{q-2} \theta_{q-1} \theta_{q}\right), \ldots$, $d\left(\theta_{1} \theta_{2} \cdots \theta_{q}\right)=d\left(\theta_{2} \cdots \theta_{q}\right)$. Hence, the dictator on $\sigma C_{n}$ is the same as the dictator on $\theta_{q} C_{n}$ which, by Step 3 , is the same as the dictator on $C_{n}$.

Step 5. In this step, we show that for all $\sigma \in \Sigma_{n}$ and all $\theta \in \Theta_{n}, d$ is a dictator on all pairs $\left\{\sigma_{1}, \sigma_{2}\right\}$ for which $\sigma_{1} \in \sigma C_{n}$ and $\sigma_{2} \in \theta \sigma C_{n}$.

First, suppose that $\left\{\sigma_{1}, \sigma_{2}\right\}$ is a free pair. If we identify $\sigma_{1}$ with $\sigma x$ in Step 2, it follows that if $\sigma_{2} \in\left\{\theta \sigma x, \theta \sigma x_{i^{*}}, \theta \sigma x_{j^{*}}\right\}$, then someone receives the same object in $\sigma_{1}$ and $\sigma_{2}$, contradicting the assumption that $\left\{\sigma_{1}, \sigma_{2}\right\}$ is a free pair. Hence, $\sigma_{2} \in E$. By choosing $\sigma_{3} \in E$ distinct from $\sigma_{2},\left\{\sigma_{1}, \sigma_{2}, \sigma_{3}\right\}$ is a free triple with $\sigma_{3} \in \theta \sigma C_{n}$. By Step $4, d$ is the dictator on $\left\{\sigma_{2}, \sigma_{3}\right\}$ and, therefore, $d$ must also be the dictator on the free triple $\left\{\sigma_{1}, \sigma_{2}, \sigma_{3}\right\}$.

If $\left\{\sigma_{1}, \sigma_{2}\right\}$ is not a free pair, $d$ is trivially a dictator on this pair if $\sigma_{1}(d)=$ $\sigma_{2}(d)$, so we only need to consider the case in which $\sigma_{1}(d) \neq \sigma_{2}(d)$. Consider an arbitrary profile $\mathbf{R} \in \Pi_{i=1}^{n} \mathcal{R}_{i A}$ for which $\sigma_{1} P_{d} \sigma_{2}$. By Step 2, there exist $\sigma_{3}, \sigma_{4} \in \theta \sigma C_{n}$ such that $\left\{\sigma_{1}, \sigma_{3}, \sigma_{4}\right\}$ is a free triple. Because someone receives the same object in $\sigma_{1}$ and $\sigma_{2}$, whereas everyone receives different objects in any two assignments in $\theta \sigma C_{n}$, the fact that $\left\{\sigma_{1}, \sigma_{3}, \sigma_{4}\right\}$ is a free triple implies that $\left\{\sigma_{2}, \sigma_{3}, \sigma_{4}\right\}$ is also a free triple. Because the two free triples have two alternatives in common, they must have the same dictator. By Step $4, d$ is the dictator on $\left\{\sigma_{2}, \sigma_{3}, \sigma_{4}\right\}$, so $d$ is the common dictator. 
Note that our assumptions imply that $d$ receives a different object in each of $\sigma_{1}, \sigma_{2}, \sigma_{3}$, and $\sigma_{4}$. Hence, there exists a profile $\mathbf{R}^{\prime} \in \Pi_{i=1}^{n} \mathcal{R}_{i A}$ for which (i) $R_{i}^{\prime}=R_{i}$ for all $i \neq d$ and (ii) $\sigma_{1} P_{d}^{\prime} \sigma_{3} P_{d}^{\prime} \sigma_{4} P_{d}^{\prime} \sigma_{2}$. Because $d$ is a dictator on each adjacent pair in this sequence of alternatives, we have $\sigma_{1} P^{\prime} \sigma_{3} P^{\prime} \sigma_{4} P^{\prime} \sigma_{2}$. Transitivity of $R^{\prime}$ implies that $\sigma_{1} P^{\prime} \sigma_{2}$. We then have $\sigma_{1} P \sigma_{2}$ by IIA. Thus, $d$ is a dictator on $\left\{\sigma_{1}, \sigma_{2}\right\}$.

Step 6. We now show that for all $\sigma, \sigma^{\prime} \in \Sigma_{n}$, if $d$ is dictator on all pairs $\left\{\sigma_{1}, \sigma_{2}\right\}$ such that $\sigma_{1} \in \sigma C_{n}$ and $\sigma_{2} \in \sigma^{\prime} C_{n}$, then for all $\theta \in \Theta_{n}$, $d$ is a dictator on all pairs $\left\{\sigma_{1}^{*}, \sigma_{2}^{*}\right\}$ such that $\sigma_{1}^{*} \in \sigma C_{n}$ and $\sigma_{2}^{*} \in \theta \sigma^{\prime} C_{n}$.

Suppose that for some admissible profile $\mathbf{R}, \sigma_{1}^{*} P_{d} \sigma_{2}^{*}$. Consider an assignment $\sigma_{3}^{*} \in \sigma^{\prime} C_{n}$ in which $d$ receives a different object than in either $\sigma_{1}^{*}$ or $\sigma_{2}^{*}$. There then exists another admissible profile $\mathbf{R}^{\prime}$ such that (i) $R_{i}^{\prime}=R_{i}$ for all $i \neq d$ and (ii) $\sigma_{1}^{*} P_{d}^{\prime} \sigma_{3}^{*} P_{d}^{\prime} \sigma_{2}^{*}$. It follows from the hypothesis of this step and from Step 5 that $\sigma_{1}^{*} P^{\prime} \sigma_{3}^{*} P^{\prime} \sigma_{2}^{*}$. Transitivity of $P^{\prime}$ then implies that $\sigma_{1}^{*} P^{\prime} \sigma_{2}^{*}$. Hence, using IIA, we conclude that $\sigma_{1}^{*} P \sigma_{2}^{*}$.

Step \%. To complete the proof that $\prod_{i=1}^{n} \mathcal{R}_{i A}$ is Arrow-inconsistent, we show that $d$ is a dictator on $\left\{\sigma, \sigma^{\prime}\right\}$ for any $\sigma, \sigma^{\prime} \in \Sigma_{n}$. The proof is trivial if $\sigma(d)=\sigma^{\prime}(d)$, so suppose that $\sigma(d) \neq \sigma^{\prime}(d)$. As in Step 4, there exist $\theta_{1}, \theta_{2}, \ldots, \theta_{q} \in \Theta_{n}$ such that $\sigma=\theta_{1} \theta_{2} \cdots \theta_{q} \sigma^{\prime}$. From Step 5, $d$ is a dictator on all pairs with one element in $\sigma^{\prime} C_{n}$ and the other in $\theta_{q} \sigma^{\prime} C_{n}$. From Step 6, $d$ is therefore a dictator on all pairs with one element in $\sigma^{\prime} C_{n}$ and the other in $\theta_{q-1} \theta_{q} \sigma^{\prime} C_{n}$. A repeated application of Step 6 leads to the conclusion that $d$ is a dictator on $\left\{\sigma, \sigma^{\prime}\right\}$.

Because the domain in the assignment problem is non-Cartesian, we could not analyze individual preference domains separately, as we have done in the Cartesian case. Nevertheless, we were able to group the alternatives in such a way that we could use Arrow's theorem "locally" on each subgroup and then use connection arguments to show that a single individual dictates on the complete set of alternatives. In the set obtained by taking all the circular permutations of an arbitrary assignment, nobody receives the same object in any of these assignments. Thus, these subgroups of alternatives, which partition $\Sigma_{n}$, are free $n$-tuples. If we take one of these subgroups, say $\sigma C_{n}$, and transpose who receives two of the objects, we obtain another one of the subgroups, say $\theta \sigma C_{n}$. A key step in the proof of Theorem 13 is that there is a free triple that overlaps these two subgroups of alternatives. These free triples play a fundamental role in the connection arguments.

Bordes and Le Breton (1990a) have used this kind of reasoning to analyze 
matching and pairing problems. In a matching problem, there are two groups of $n$ individuals and each person from one group is matched with a person from the other group. The classic example of a matching problem is the marriage problem in which a group of men and women form married couples. In a pairing problem, there is a single set of $2 n$ individuals who are grouped in pairs. For example, if college dorm rooms are designed for two people, we have a pairing problem. Provided that individuals only care about who they are matched with, but otherwise have unrestricted preferences, the preference domain in the matching problem is Arrow-inconsistent if $n \geq 4$ and the preference domain in the pairing problem is Arrow-inconsistent if $n \geq 2$. See Bordes and Le Breton (1990a) for the details.

\subsection{Exchange economies}

The set of feasible allocations in an exchange economy is another example of a non-Cartesian set of alternatives. In an exchange economy, there is a fixed aggregate endowment $\omega \in \mathbb{R}_{++}^{m}$ of $m$ goods to be distributed among $n$ individuals. We assume that $m \geq 2$ and $n \geq 2$. When there is no free disposal of goods, the set of feasible allocations for this economy is $E(\omega)=$ $\left\{x \in \mathbb{R}_{+}^{m n} \mid \sum_{i=1}^{n} x_{j}^{i}=\omega_{j}\right.$ for all $\left.j=1, \ldots, m\right\}$. Let $E^{\circ}(\omega)$ denote the interior of this set; i.e., $E^{\circ}(\omega)$ is the set of feasible allocations in which everybody's consumption of each good is positive.

Individual preferences are defined over the set of all possible allocations $\mathbb{R}_{+}^{m n}$, not just the feasible allocations in $E(\omega)$. As a preference domain, we consider the classical private goods preference domain $\mathcal{C}_{p r}$ introduced in Section 7. Recall that this is the set of all profiles of selfish, continuous, strictly monotonic in own consumption, convex individual preferences.

We know from Example 18 that a social welfare function $F: \mathcal{C}_{p r} \rightarrow \mathcal{R}$ can satisfy all three of the Arrow axioms when $X=\mathbb{R}_{+}^{m n}$. However, we also know from Theorem 10 and Examples 19 and 20 that the restriction of $F$ to the set of allocations in which everybody has a positive consumption of all goods or to the set of allocations in which everybody has a positive consumption of some good cannot satisfy all of the Arrow axioms. Bordes, Campbell, and Le Breton (1995) have shown that this impossibility also holds for the restriction of $F$ to $E^{\circ}(\omega)$. Equivalently, if we let $X=E^{\circ}(\omega)$ and $\mathcal{D}$ be the restriction of $\mathcal{C}_{p r}$ to $X$, then $\mathcal{D}$ is an Arrow-inconsistent preference domain. ${ }^{71}$

\footnotetext{
${ }^{71}$ Using a Border-type example (see Example 18), one can show that the restriction of
} 
Theorem 14. If $X=\mathbb{R}_{+}^{m n}$ with $m \geq 2$ and if $\omega \in \mathbb{R}_{++}^{m}$, there is no social welfare function with a classical private goods preference domain whose restriction to $E^{\circ}(\omega)$ satisfies IIA, WP, and ND. ${ }^{72}$

To prove this theorem, Bordes, Campbell, and Le Breton used a version of the local approach, but they had to depart quite significantly from the conventional arguments described in earlier sections. To see why this is necessary, we prove Theorem 14 for the case in which $m=n=2$; i.e., for an Edgeworth box economy.

Proof (for $m=n=2$ ). Step 1. Reasoning as in Examples 8 and 17, $\{x, y\}$ is a free pair for person $i$ if and only if neither $x^{i}>y^{i}$ nor $y^{i}>x^{i}{ }^{i 3}$ Because of the resource constraint, if a pair is free for one individual, it is also free for the other individual. Any pair that is not free is trivial, with person $i$ having a strict preference for $x$ over $y$ if and only if person $j$ has the reverse strict preference. It is not possible for anyone to be indifferent on a trivial pair.

Step 2. Consider a triple of alternatives $\{x, y, z\}$ in $E^{\circ}(\omega)$. The discussion in Step 2 of Example 8 shows that for this triple to be free for individual one, the alternatives must be situated as shown in Figure 11. ${ }^{74}$ This triple is not free for person two because $y$ cannot be ranked last in this triple by this individual. More generally, if $\{x, y, z\}$ is a free triple for person $i$, then there is an alternative $a \in\{x, y, z\}$ with the property that $j$ can have any preference on this triple in which $a$ is strictly preferred to one of the other two alternatives. In Figure 11, $a=y$. If the three alternatives are arranged in increasing order of the consumption of good one, this implies that $j$ has a single-peaked preference on this triple. Thus, there are no free triples in $E^{\circ}(\omega)$, which is why the local analysis must be modified in order for it to apply to an Edgeworth box economy.

Step 3. For any free pair $\{x, y\}$ and any $i \in\{1,2\}$, we can find a third alternative $z \in E^{\circ}(\omega)$ such that $\{x, y, z\}$ is a free triple for $i$ and the alter-

$\mathcal{C}_{p r}$ to $E(\omega)$ is Arrow-consistent. Note that if a preference domain is Arrow-inconsistent, it does not follow that the restriction of the preference domain to a proper subset of the alternatives is also Arrow-inconsistent. See Section 1 of Bordes, Campbell, and Le Breton (1995) for an example of this phenomenon.

${ }^{72}$ Bordes, Campbell, and Le Breton (1995) assumed that preferences are strictly convex. Their arguments also apply to convex preferences because the same pairs and triples of alternatives are strictly free with either convexity assumption.

${ }^{73} \mathrm{In}$ our discussion of the two-person case, we use $i$ to denote an arbitrary member of $\{1,2\}$ and $j$ to denote the other person.

${ }^{74}$ In Figures 11 and 12, $O_{1}$ (resp. $O_{2}$ ) is person one's (resp. two's) origin. 
native $a$ that cannot be ranked last by the other individual is a prespecified member of the triple. For example, if $i=1$, for the $x$ and $y$ depicted in Figure 1, $z$ would need to be located in region III if $a=x$, in region IX if $a=y$, and in region VI if $a=z$.

Step 4. Suppose that $F$ is a social welfare function with a classical private goods preference domain whose restriction to $E^{\circ}(\omega)$ satisfies IIA and WP. The next part of the proof is concerned with determining the decisiveness implications of these assumptions. In this two-person economy, individual $i$ is almost decisive for $(x, y)$ if $x$ is socially preferred to $y$ when $i$ prefers $x$ to $y$ and $j$ prefers $y$ to $x$. Let $A D_{i}(x, y)$ denote that $i$ is almost decisive for $(x, y)$. Suppose that $\{x, y, z\}$ is a free triple for $i$ and, without loss of generality, that $y$ is the alternative in this triple that cannot be ranked last by $j$. In this step, we show that either $i$ is almost decisive on all ordered pairs of distinct alternatives in $\{x, y, z\}$ or $j$ is almost decisive on all ordered pairs $(a, b)$ of distinct alternatives in $\{x, y, z\}$ for which $b \neq y .{ }^{75}$

Let $\mathbf{R}^{1}$ be a profile for which $x P_{i}^{1} y P_{i}^{1} z$ and $y P_{j}^{1} z P_{j}^{1} x$. By WP, we have $y P^{1} z$. If $z R^{1} x$ and $x R^{1} y, R^{1}$ would not be an ordering. Therefore, either $x P^{1} z$ or $y P^{1} x$. We consider each case in turn.

Case 1. If $x P^{1} z$, IIA implies $A D_{i}(x, z)$. Suppose now that $A D_{i}(a, b)$ holds for $a, b \in\{x, y, z\}$ with $a \neq y$. Let $c$ be the other alternative in the triple and consider a profile $\mathbf{R}^{2}$ for which $a P_{i}^{2} b P_{i}^{2} c$ and $b P_{j}^{2} c P_{j}^{2} a$. We then have $a P^{2} b$ by $A D_{i}(a, b)$ and $b P^{2} c$ by WP. Transitivity of $R^{2}$ then implies that $a P^{2} c$ and, hence, $A D_{i}(a, c)$. A similar argument can be used to show that $A D_{i}(c, b)$ holds. Letting $a=x$ and $b=z$, it then follows from $A D_{i}(x, z)$ that $A D_{i}(x, y)$ and $A D_{i}(y, z)$. Now letting $a=x$ and $b=y$, it follows from $A D_{i}(x, y)$ that $A D_{i}(z, y)$. Similarly, it can be shown that $A D_{i}(z, x)$ and $A D_{i}(y, x)$ both hold. Therefore, $i$ is almost decisive on all of the distinct ordered pairs in $\{x, y, z\}$.

Case 2. If $y P^{1} x$, IIA implies $A D_{j}(y, x)$. Consider a profile $\mathbf{R}^{3}$ for which $x P_{i}^{3} z P_{i}^{3} y$ and $y P_{j}^{3} x P_{j}^{3} z$. We then have $y P^{3} x$ by $A D_{j}(y, x)$ and $x P^{3} z$ by WP. Thus, $y P^{3} z$ by the transitivity of $R^{3}$, which establishes $A D_{j}(y, z)$. We can similarly show that $A D_{j}(z, x)$ and $A D_{j}(x, z)$ both hold. Therefore, $j$ is almost decisive on all ordered pairs $(a, b)$ of distinct alternatives in $\{x, y, z\}$ for which $b \neq y{ }^{76}$

\footnotetext{
${ }^{75} \mathrm{~A}$ more complete proof of this result may be found in the proof of Lemma 1 in Bordes, Campbell, and Le Breton (1995).

${ }^{76}$ Note that none of the profiles used in this demonstration has person $j$ ranking $y$ last in $\{x, y, z\}$.
} 
Step 5. Suppose that Case 2 of the preceding step holds. By Step 3, there exists a $w \in E^{\circ}(\omega)$ such that $\{w, x, y\}$ is a free triple for $j$ with $w$ never ranked last by $i$ in this triple. If $A D_{i}(x, y)$ holds, we would have a contradiction with $A D_{j}(y, x)$. Hence, by Step $4, j$ is almost decisive on all of the distinct ordered pairs in $\{w, x, y\}$. In particular, we have $A D_{j}(x, y)$. A similar argument can be used to show that $A D_{j}(z, y)$. Thus, Steps 4 and 5 show that if $\{x, y, z\}$ is a free triple for $i$, then either $i$ or $j$ is almost decisive on every ordered pair of distinct alternatives in $\{x, y, z\}$.

Step 6. For the triple considered in the previous two steps, suppose that $A D_{i}(x, y)$ holds. Consider a profile $\mathbf{R}^{4}$ for which $x P_{i}^{4} y P_{i}^{4} z, y P_{j}^{4} x$, and $y P_{j}^{4} z$, with $x$ and $z$ ranked arbitrarily by $j$. We then have $x P^{4} y$ by $A D_{i}(x, y)$ and $y P^{4} z$ by WP. Transitivity of $R^{4}$ implies that $x P^{4} z$, so $i$ is decisive for $(x, z)$. Similar arguments can be used to show that one of the two individuals is decisive on all ordered pairs of distinct alternatives in $\{x, y, z\}$. In other words, there is a dictator on this triple.

Step 7. Because there are no free triples, we cannot connect two nontrivial pairs with a sequence of overlapping free triples, as in the local approach. Nevertheless, it is possible to "connect" these pairs with a sequence of overlapping triples, with each triple in the sequence free for one of the two individuals. As in the local approach, overlapping triples have two alternatives in common. As a consequence, who is the dictator on each triple must be the same. Hence, one individual, say $d$, dictates on all the nontrivial pairs.

We illustrate the connection argument with the nontrivial pairs $\{u, v\}$ and $\{x, y\}$ shown in Figure 12. For the $w$ and $z$ shown in Figure 12, the sequence of triples $\{u, v, z\},\{v, z, w\},\{z, w, x\}$, and $\{w, x, y\}$ exhibits the properties we require. The first two triples in this sequence are free for person two, while the other two are free for person one. ${ }^{77}$

Step 8. A separation argument is now used to show that $d$ is also a dictator on the trivial pairs. Suppose that $\{x, y\}$ is a trivial pair and that $x P_{d} y$. As in our discussion of Example 19, $\{x, y\}$ is separable for $d$, so there

\footnotetext{
${ }^{77}$ Viewed from person one's perspective, $u, v, x$, and $y$ have the same relative positions as the alternatives used in Figure 3. In that diagram, the alternatives $w$ and $z$ were chosen to lie on the axes sufficiently far from the origin so that all the triples in the sequence are free for this individual. That argument is unaffected if $w$ and $z$ are modified slightly so that the consumption of both goods is positive. However, our earlier argument made essential use of the assumption that the set of alternatives is unbounded from above. In Figure 12, if $z$ were instead chosen to lie above the line through $u$ and $v$, but below the horizontal line through $y$ (as in Figure 3), $z$ would lie outside the Edgeworth box.
} 
exists a $z \in E^{\circ}(\omega)$ such that $\{x, z\}$ and $\{y, z\}$ are free for $d$ and there exists a profile $\mathbf{R}^{\prime}$ in the domain for which $x P_{d}^{\prime} z P_{d}^{\prime} y$. Because $d$ is a dictator on the two free pairs, we have $x P^{\prime} z$ and $z P^{\prime} y$. Hence, $x P^{\prime} y$ by transitivity. IIA now implies that $x P y$, which completes the proof.

The proof of Theorem 14 is completed by first showing that the theorem holds for $n \geq 3$ and $m=2$, and then proving that the two-good impossibility can be extended to more goods. The proof of the two-good case makes use of Redekop's topological version of the local approach discussed in Section 6. Unlike the two-person case we have considered in detail, when there are at least three individuals, any free pair can be embedded in a free triple. See Bordes, Campbell, and Le Breton (1995) for the details.

In Example 19, we have seen that when $X=\left(\mathbb{R}_{+}^{m} \backslash\left\{0_{m}\right\}\right)^{n}$ with $m \geq 2$, the domain of classical private goods preferences $\mathcal{C}_{p r}$ is hypersaturating and, hence, Arrow-inconsistent. Let $\mathcal{C}_{p r}^{=}$denote the subset of $\mathcal{C}_{p r}$ in which everyone has the same preference for own consumption in each profile. In Section 9, we noted that Redekop (1993a) has shown that $\mathcal{C}_{p r}^{=}$is also Arrow-inconsistent when $X=\left(\mathbb{R}_{+}^{m} \backslash\left\{0_{m}\right\}\right)^{n}$. These conclusions also hold if $X=\mathbb{R}_{++}^{m n}$. In view of the impossibility result established in Theorem 14 for classical private goods preferences, one might wonder if the restriction of $\mathcal{C}_{p r}^{=}$to the interior of the set of feasible allocations for an exchange economy is also Arrow-inconsistent. Example 22 demonstrates that this is not the case. This example illustrates the importance of the assumption that the set of alternatives is Cartesian for Redekop's impossibility theorem.

Example 22. In this example, the set of alternatives is $E^{\circ}(\omega)$, where $\omega \in$ $\mathbb{R}_{++}^{m}$ and $m \geq 2$. Preferences are defined on $\mathbb{R}_{+}^{m n}$. The domain $\mathcal{D}$ of the social welfare function is the restriction of $\mathcal{C}_{p r}^{=}$to $E^{\circ}(\omega)$. We show that this domain is Arrow-consistent.

A social welfare function $F: \mathcal{D} \rightarrow \mathcal{R}$ satisfying the three Arrow axioms can be constructed as follows. Let $e$ denote the alternative in which each person receives an equal share of each good. For each profile $\mathbf{R} \in \mathcal{D}$, (i) $e$ is socially preferred to all of the other alternatives in $E^{\circ}(\omega)$ and (ii) for all pairs of alternatives that do not include $e$, the social ranking coincides with $R_{1}$. Because $R_{1}$ is an ordering, $F(\mathbf{R})$ is as well. By construction, $F$ satisfies IIA. Person 1 never ranks $e$ first in $E^{\circ}(\omega)$, so $F$ satisfies ND. Because everyone has the same preferences, $e$ is Pareto optimal for all $\mathbf{R} \in \mathcal{D}$. This observation combined with the way social preferences are defined on pairs that do not 
include $e$ implies that $F$ satisfies $\mathrm{WP}^{78}$

\section{Effective social welfare functions}

Determining a social preference is often just the first step of a social choice procedure whose ultimate goal is to choose a set of alternatives. Given a preference profile, the social welfare function first aggregates the individual preferences into a social preference and then this social preference is used to determine the socially-best alternatives in the feasible set of alternatives. In this section, we consider the implications of requiring socially-best alternatives to exist on some collections of subsets of the universal set of alternatives when there are private alternatives.

For a nonempty set $A \subseteq X$ and an ordering $R \in \mathcal{R}$, the set of best alternatives in $A$ according to $R$ is

$$
B(A, R)=\{x \in A \mid x R y \text { for all } y \in A\} .
$$

Let $\mathcal{A}$ be a collection of nonempty subsets of $X$. A social welfare function $F: \mathcal{D} \rightarrow \mathcal{R}$ is effective on $\mathcal{A}$ if for all $\mathbf{R} \in \mathcal{D}$ and all $A \in \mathcal{A}, B(A, F(\mathbf{R})) \neq \emptyset$. For the classical domain of private goods preferences used in Example 17, Campbell (1989a) has shown that there is no social welfare function that is both effective on all compact subsets of $X$ and satisfies the Arrow axioms.

Theorem 15. If $X=\mathbb{R}_{+}^{m n}$ with $m \geq 2$, there is no social welfare function with a classical private goods preference domain that is effective on the nonempty compact subsets of $X$ and satisfies IIA, WP, and ND.

Proof. On the contrary, suppose that there exists a social welfare function that satisfies all the assumptions of the theorem. From our discussion of Example 19, we know that there must be a dictator $d$ on $\left(\mathbb{R}_{+}^{m} \backslash\left\{0_{m}\right\}\right)^{n}$. Given the restrictions on individual preferences, in order for $d$ not to be a dictator on all of $X$, there must exist $i \in N, x, y \in X$ with $x^{d} \neq 0_{m}$ and $y^{i}=0_{m}$, and a profile $\mathbf{R} \in \mathcal{D}$ such that $x P_{d} y$, but $y R x$. Consider the alternative $z$ obtained by adding $\varepsilon>0$ to each person's consumption of every good in $y$. Because $R_{d}$ is continuous, for sufficiently small $\varepsilon$, we have $x P_{d} z P_{d} y$. Let $A=\{x, y, z\}$. Because $d$ is a dictator on $\{x, z\}, z \notin B(A, R)$. By WP,

\footnotetext{
${ }^{78}$ Note that the construction in this example can be used to show that a preference domain is Arrow-consistent whenever there is an alternative that is in the Pareto set for all admissible preference profiles.
} 
$y \notin B(A, R)$. Because the social welfare function is effective on compact sets, we therefore have $B(A, R)=\{x\}$, which contradicts the assumption that $y R x$.

The requirement that socially-preferred alternatives exist on all compact subsets of $X$ may be too demanding. For example, we might only need to choose out of compact, comprehensive subsets of $X$. A set $A \subseteq \mathbb{R}_{+}^{m}$ is comprehensive if for any $x, y \in \mathbb{R}_{+}^{m}$ with $x \geq y, x \in A$ implies $y \in A$. For any $x \in \mathbb{R}_{+}^{m}$, the comprehensive set of $x$ is $x_{\downarrow}=\left\{y \in \mathbb{R}_{+}^{m} \mid x \geq y\right\}$. Following Duggan (1996), for any $x, y \in \mathbb{R}_{+}^{m}, x_{\downarrow} \cup y_{\downarrow}$ is called the corner set of $\{x, y\}$.

By strengthening WP to SP in Theorem 15, we obtain an impossibility theorem when the social welfare function is only required to be effective on the compact, comprehensive subsets of $X$.

Theorem 16. If $X=\mathbb{R}_{+}^{m n}$ with $m \geq 2$, there is no social welfare function with a classical private goods preference domain that is effective on the nonempty compact, comprehensive subsets of $X$ and satisfies IIA, SP, and ND.

Proof. The proof of this theorem is similar to the proof of Theorem 15. Instead of letting $A=\{x, y, z\}$, we now let $A=x_{\downarrow} \cup z_{\downarrow}$. By SP, $B(A, R) \subseteq$ $\{x, z\}$. As in the earlier proof, $B(A, R)=\{x\}$, which results in a contradiction with the assumption that $y R x$.

Donaldson and Weymark (1988) have noted that Border's social welfare function in Example 18 is not effective for standard feasible sets of allocations because there is always a discontinuity in the social preference on the boundary of the allocation space. For example, consider a two-person, twogood exchange economy. Because the social welfare function satisfies WP, we can restrict our search for socially-best alternatives to allocations in the Edgeworth box for this economy. For the social welfare function in Example 18, all points in the Edgeworth box excluding the two origins are socially preferred to person two's origin which, in turn, is socially preferred to person one's origin. However, for all points other than the two origins, transferring some of any good from person one to person two is a social improvement (provided person one remains with some consumption). Hence, there is no socially-best alternative.

Bone (2003) has shown that effectiveness has strong implications for an exchange economy. As in Section 10.2, let $E(\omega)$ be the feasible allocations 
in an $m$-good, $n$-person exchange economy when there is no free disposal of goods. Suppose that $F$ is a social welfare function with a classical private goods preference domain that is effective on $E(\omega)$ and that satisfies IIA and WP. By Theorem 14, there is a dictator $d$ on $E^{\circ}(\omega)$ (the interior of $E(\omega)$ ). Because $d$ has no best alternative in $E^{\circ}(\omega)$, for any admissible profile, any socially-best alternative in $E(\omega)$ must give someone nothing of at least one good. Bone has shown that, in fact, someone consumes the whole endowment.

Requiring a social welfare function to be effective on the compact subsets of $X$ is less demanding than requiring social preferences to be continuous. Variants of Theorems 15 and 16 can be established quite simply when it is assumed that social preferences are continuous on $X .{ }^{79}$ From our discussion of Example 20, we know that there must be a dictator on the interior of $\mathbb{R}_{+}^{m n}$ if the social welfare function satisfies IIA and WP. Continuity of the social preference extends this dictatorial power to all of $\mathbb{R}_{+}^{m n}$.

In view of the largely negative results surveyed in Part I, it is natural to wonder if more satisfactory social choice procedures are possible if we abandon the objective of constructing a social welfare function and, instead, directly determine the socially-best alternatives in an agenda as a function of the individual preferences. This is the subject of Part II.

\section{Part II: Social choice correspondences}

\section{A choice-theoretic version of Arrow's theorem}

\subsection{The Arrow choice axioms}

An agenda is a nonempty subset of $X$. The set of admissible agendas, the agenda domain, is $\mathcal{A}$, a collection of nonempty subsets of $X$. Each agenda in the agenda domain is a potential feasible set.

A social choice correspondence $C: \mathcal{A} \times \mathcal{D} \rightarrow X$ is a mapping that assigns a nonempty subset of the agenda to each admissible agenda and admissible profile. ${ }^{80}$ That is, for all $A \in \mathcal{A}$ and all $\mathbf{R} \in \mathcal{D}, \emptyset \neq C(A, \mathbf{R}) \subseteq A$. The

\footnotetext{
${ }^{79}$ See, for example, Campbell $(1992 b ; 1996)$ for discussions of continuity of social preference.

${ }^{80}$ The assumption that the domain of $C$ is the Cartesian product of $\mathcal{A}$ and $\mathcal{D}$ excludes from consideration the possibility that the feasibility of an agenda may depend on the
} 
set $C(A, \mathbf{R})$ is called the choice set. If, for all agendas and profiles in the domain, the choice set only contains a single alternative, then $C$ is a social choice function.

Choice-theoretic versions of Arrow's Theorem employ a choice consistency condition. A choice consistency condition for a social choice correspondence places restrictions on how the choices from different agendas are related for a given preference profile. The choice consistency axiom used in Arrow's Theorem is Arrow's Choice Axiom.

Arrow's Choice Axiom (ACA). For all $A^{1}, A^{2} \in \mathcal{A}$ and all $\mathbf{R} \in \mathcal{D}$, if $A^{1} \subset A^{2}$ and $C\left(A^{2}, \mathbf{R}\right) \cap A^{1} \neq \emptyset$, then $C\left(A^{1}, \mathbf{R}\right)=C\left(A^{2}, \mathbf{R}\right) \cap A^{1}$.

In words, for a given profile $\mathbf{R}$, if anything that is chosen in $A^{2}$ is feasible when the agenda shrinks to $A^{1}$, then the choice set for the smaller agenda consists of that part of the original choice set that is still feasible.

The definition of a social welfare function incorporates the social rationality condition that all social rankings of the alternatives are orderings. For the agenda domain used in our version of Arrow's Theorem for social choice correspondences, ACA is the choice-theoretic analogue of this social rationality condition. To see why, we need to take a brief excursion into revealed preference theory.

A social welfare function $F: \mathcal{D} \rightarrow \mathcal{R}$ rationalizes the social choice correspondence $C$ if for all $A \in \mathcal{A}$ and all $\mathbf{R} \in \mathcal{D}, C(A, \mathbf{R})=B(A, F(\mathbf{R}))$. That is, for each agenda $A$ and each profile $\mathbf{R}$ in the domain of $C$, the choice set consists of the best elements in $A$ according to the social preference relation $R$ assigned to the profile $\mathbf{R}$ by the social welfare function $F$.

For each $\mathbf{R} \in \mathcal{D}$, the social choice correspondence $C$ defines a choice correspondence $C_{\mathbf{R}}: \mathcal{A} \rightarrow X$. Revealed preference theory has identified a number of circumstances in which a choice correspondence can be rationalized by an ordering. See, for example, Suzumura (1983). These results can be trivially reformulated as social choice revealed preference theorems. ${ }^{81}$

In the version of Arrow's Theorem that we present in the next section, we assume that the agenda domain is the set of all finite subsets of $X{ }^{82}$

preference profile, as in asymmetric information models with self-selection constraints.

${ }^{81}$ The revealed preference results described below were originally established for choice correspondences.

${ }^{82}$ The inclusion of the agendas containing only one alternative is of no consequence because the choice on one-alternative agendas is completely determined by the requirement 
Complete Finite Agenda Domain. $A \in \mathcal{A}$ if and only if $A$ is a finite subset of $X$.

For this agenda domain, Arrow (1959) has shown that a social choice correspondence $C$ can be rationalized by a social welfare function if and only if $C$ satisfies ACA. It is this revealed preference theorem that provides the link between ACA and the social rationality condition used in his social welfare function impossibility theorem.

Theorem 17. On a complete finite agenda domain, a social choice correspondence can be rationalized by a social welfare function if and only if it satisfies ACA.

With this agenda domain, the social welfare function $F$ that rationalizes a social choice correspondence $C$ satisfying ACA is uniquely determined by the choices made in the binary agendas. That is, for all $x, y \in X$ and all $\mathbf{R} \in \mathcal{D}, x F(\mathbf{R}) y$ if and only if $x \in C(\{x, y\}, \mathbf{R})$. For a fixed profile, the binary relation defined in this way is called the base relation.

Arrow's revealed preference theorem is a special case of a theorem due to Hansson (1968) that has played an important role in the development of some of the results described in subsequent sections. In the version of Hansson's Theorem that we consider, the agenda domain is closed under finite unions. An agenda domain $\mathcal{A}$ is closed under finite unions if for all $A^{1}, A^{2} \in \mathcal{A}$, $A^{1} \cup A^{2} \in \mathcal{A}$. A complete finite agenda domain is closed under finite unions.

Theorem 18. A social choice correspondence whose agenda domain is closed under finite unions can be rationalized by a social welfare function if and only if it satisfies $\mathrm{ACA}{ }^{83}$

If some of the binary agendas are not feasible, the social welfare function that rationalizes the social choice correspondence need not be unique. ${ }^{84}$

Arrow's Choice Axiom is closely related to the Weak Axiom of Revealed Preference. For a given profile, this axiom requires that if it is ever the case

that the choice sets are nonempty. In all of the agenda domains we consider, it is implicitly assumed that only nonempty sets are in the domain.

${ }^{83}$ This theorem is a special case of a more general result established by Hansson.

${ }^{84}$ Arrow (1951, p. 20) has said that even when no binary agendas are in the agenda domain, "under certain plausible conditions" it is nevertheless possible to rationalize a choice correspondence. However, he does not say what these conditions are. 
that $x$, but not $y$, is chosen when both $x$ and $y$ are feasible, then $y$ is never chosen when $x$ is in the agenda.

Weak Axiom of Revealed Preference (WARP). For all $x, y \in X$ and all $\mathbf{R} \in \mathcal{D}$, if there exists an $A^{1} \in \mathcal{A}$ such that $x \in C\left(A^{1}, \mathbf{R}\right)$ and $y \in A^{1} \backslash C\left(A^{1}, \mathbf{R}\right)$, then there does not exist an $A^{2} \in \mathcal{A}$ such that $y \in C\left(A^{2}, \mathbf{R}\right)$ and $x \in A^{2}$.

If a social choice correspondence satisfies WARP, then it necessarily satisfies ACA. In general, the reverse implication does not hold. However, if the agenda domain is closed under finite unions, WARP is equivalent to ACA. See Suzumura (1983). Because a complete finite agenda domain is closed under finite unions, it does not matter which of these two axioms is used in the choice-theoretic version of Arrow's Theorem. However, on most of the agenda domains we consider in subsequent sections, these axioms are not equivalent. When this is the case and the domain has an economic interpretation, it has been customary to use ACA rather than WARP.

Choice consistency conditions link the choices made from different agendas for a fixed profile. In contrast, independence conditions link the choices made from a fixed agenda for different profiles. Independence of Infeasible Alternatives requires the choice set to be independent of preferences over alternatives not in the agenda.

Independence of Infeasible Alternatives (IIF). For all $A \in \mathcal{A}$ and all $\mathbf{R}^{1}, \mathbf{R}^{2} \in$ $\mathcal{D}$, if $\mathbf{R}^{1}$ and $\mathbf{R}^{2}$ coincide on $A$, then $C\left(A, \mathbf{R}^{1}\right)=C\left(A, \mathbf{R}^{2}\right)$.

It might seem that the natural choice-theoretic analogue to Independence of Irrelevant Alternatives would only place restrictions on the choices from agendas containing only two alternatives. However, a binary version of IIF is vacuous if no binary agendas are in the agenda domain, as is typically the case in economic problems.

Plott (1976) has argued that almost any reasonable social decision procedure satisfies IIF. In essence, his argument is that a necessary condition for a social choice rule to be implementable (using any of the standard cooperative or noncooperative solution concepts) is that the alternatives chosen must only depend on individual preferences for feasible alternatives. In this view, the independence assumption is not a normative principle of social choice, but is, rather, a constraint on what is achievable. ${ }^{85}$ However, not everyone believes that implementability is a necessary requirement for a social

\footnotetext{
${ }^{85}$ See Campbell (1992a; 1992c) for further discussion of the relationship between IIF and
} 
choice correspondence to be satisfactory. Implementability is a demanding requirement that is not satisfied by, for example, the standard Walrasian competitive equilibrium correspondence on standard economic domains.

We consider weak and strong versions of the Pareto principle. For all $A \in \mathcal{A}$ and all $\mathbf{R} \in \mathcal{D}$, the weak Pareto set (the set of weakly Pareto optimal alternatives) is

$$
\mathcal{P}^{w}(A, \mathbf{R})=\left\{x \in A \mid \nexists y \in A \text { such that } y P_{i} x \text { for all } i \in N\right\}
$$

and the strong Pareto set (the set of strongly Pareto optimal alternatives) is

$$
\begin{gathered}
\mathcal{P}^{s}(A, \mathbf{R})=\left\{x \in A \mid \nexists y \in A \text { such that } y R_{i} x \text { for all } i \in N\right. \text { and } \\
\left.y P_{j} x \text { for some } j \in N\right\} .
\end{gathered}
$$

The Weak Pareto (resp. Strong Pareto) axiom requires the choice set to be a subset of the weak (resp. strong) Pareto set. ${ }^{86}$

Weak Pareto (WP). For all $A \in \mathcal{A}$ and all $\mathbf{R} \in \mathcal{D}, C(A, \mathbf{R}) \subseteq \mathcal{P}^{w}(A, \mathbf{R})$.

Strong Pareto (SP). For all $A \in \mathcal{A}$ and all $\mathbf{R} \in \mathcal{D}, C(A, \mathbf{R}) \subseteq \mathcal{P}^{s}(A, \mathbf{R})$.

Pareto conditions place no cross-profile or cross-agenda restrictions on a social choice correspondence.

A social choice correspondence is dictatorial if the choice set is always a subset of some single individual's best alternatives in the agenda. Formally, an individual $d \in N$ is a dictator for the social choice correspondence $C$ if $C(A, \mathbf{R}) \subseteq B\left(A, R_{d}\right)$ for all $A \in \mathcal{A}$ and all $\mathbf{R} \in \mathcal{D}$. If $C(A, \mathbf{R})=B\left(A, R_{d}\right)$ for all $A \in \mathcal{A}$, then $d$ is a strong dictator for $C$. Nondictatorship is the requirement that there be no dictator.

implementability. As in Plott (1976), Campbell argues that IIF is a necessary condition for the implementabilty of a social choice correspondence. Campbell assumes that the social choice correspondence $C$ is generated from a social welfare function $F$ by setting $C(A, \mathbf{R})$ equal to the set of alternatives that are maximal in $A$ for $F(\mathbf{R})$. He has shown that if the preference domain satisfies a regularity condition and if social preferences are continuous, then $C$ satisfies IIF if and only if $F$ satisfies IIA when the agenda domain consists of all $m$-element subsets of $X$, where $m>1$.

${ }^{86}$ Some of our axioms (and acronyms) for social choice correspondences have the same names as the corresponding axioms for social welfare functions. It is always clear from the context whether a social choice correspondence or a social welfare function axiom is being used. 
Nondictatorship (ND). There is no dictator.

The Arrow axioms for a social choice correspondence are ACA, IIF, WP, and ND.

If it is possible to find a social choice correspondence that satisfies all of the Arrow axioms on the domain $\mathcal{A} \times \mathcal{D}$, it is natural to enquire if there are other desirable properties that can be satisfied on this domain. ND precludes any individual from having complete power to determine the choice set in all circumstances, but it does not rule out having one individual's preferred choices being made in almost all choice situations, nor does it preclude the decision-making power being concentrated in the hands of as few as two individuals. It is therefore of interest to see if it is possible to spread the decision-making power more widely among the population. One way that has been proposed to do this is to require the social choice correspondence to satisfy Anonymity, the requirement that the choice set from a given agenda be invariant to permutations of the individual preferences.

Anonymity (ANON). For all $A \in \mathcal{A}$ and all $\mathbf{R}^{1}, \mathbf{R}^{2} \in \mathcal{D}$, if $\mathbf{R}^{1}$ is a permutation of $\mathbf{R}^{2}$, then $C\left(A, \mathbf{R}^{1}\right)=C\left(A, \mathbf{R}^{2}\right)$.

This formulation of an anonymity condition is only appropriate if all of the individual preference domains are the same. If there are private alternatives and everyone is selfish, ANON is vacuous because if we, say, permute $i$ 's and $j$ 's preferences, $i$ would then only care about $j$ 's consumption and vice versa, which takes us outside the preference domain. For Cartesian sets of alternatives and selfish individual preference domains, it is the induced private preferences $Q_{i}$ on the individual consumptions sets $X_{i}$ that should be permuted, not the preferences on $X$.

Private Alternatives Anonymity (PANON). For all $A \in \mathcal{A}$ and all $\mathbf{R}^{1}, \mathbf{R}^{2} \in$ $\mathcal{D}$, if $\mathbf{Q}^{1}=\left(Q_{1}^{1}, \ldots, Q_{n}^{1}\right)$ is a permutation of $\mathbf{Q}^{2}=\left(Q_{1}^{2}, \ldots, Q_{n}^{2}\right)$, then $C\left(A, \mathbf{R}^{1}\right)=C\left(A, \mathbf{R}^{2}\right)$.

In the case of public alternatives, provided that the preference domain is not too degenerate, ANON is a strengthening of ND. Similarly, for private alternatives, provided that the induced private preference domain is not too degenerate, PANON is also more demanding than ND. 


\subsection{Arrow's theorem}

The version of Arrow's Theorem that we consider in this section supposes that the preference domain is unrestricted and that the agenda domain is the set of all nonempty finite subsets of $X$. With these domain assumptions, the choice-theoretic version of Arrow's Theorem shows that there is no social choice correspondence that satisfies ACA, IIF, WP, and ND if there are at least three alternatives. We provide a proof of this theorem because the proof strategy has proved useful on restricted domains as well.

Theorem 19. If $|X| \geq 3$, there is no social choice correspondence with a complete finite agenda domain and an unrestricted preference domain that satisfies ACA, IIF, WP, and ND.

Proof. On the contrary, suppose that there exists a social correspondence $C: \mathcal{A} \times \mathcal{D} \rightarrow X$ that satisfies all the assumptions of the theorem. By Theorem $17, C$ can be rationalized by a social welfare function $F: \mathcal{D} \rightarrow \mathcal{R}$. Further, for each profile $\mathbf{R}$ in the preference domain, the rationalizing social ordering $F(\mathbf{R})$ is uniquely given by the base relation.

Consider any $x, y \in X$ and any $\mathbf{R}^{1}, \mathbf{R}^{2} \in \mathcal{D}$ that coincide on $\{x, y\}$. By IIF, $C\left(\{x, y\}, \mathbf{R}^{1}\right)=C\left(\{x, y\}, \mathbf{R}^{2}\right)$ and, hence, because $F$ is constructed from the base relations, $x F\left(\mathbf{R}^{1}\right) y$ if and only if $x F\left(\mathbf{R}^{2}\right) y$. Thus, $F$ satisfies IIA.

Next, consider any $x, y \in X$ and any $\mathbf{R} \in \mathcal{D}$ for which $x P_{i} y$ for all $i \in N$. Because $C$ satisfies WP, $C(\{x, y\}, \mathbf{R})=\{x\}$. Because $F(\mathbf{R})$ rationalizes $C$, $x$ is socially preferred to $y$ according to $F(\mathbf{R})$; i.e., $F$ satisfies WP.

By the social welfare function version of Arrow's Theorem, $F$ is dictatorial. Let $d \in N$ be this dictator. Because $C$ is nondictatorial, there exists an agenda $A \in \mathcal{A}$, alternatives $x, y \in A$, and a profile $\mathbf{R} \in \mathcal{D}$ such that $x P_{d} y$ and $y \in C(A, \mathbf{R})$. ACA then implies that $y \in C(\{x, y\}, \mathbf{R})$. Thus, $y F(\mathbf{R}) x$, contradicting the assumption that $d$ is a dictator for $F$.

\section{Unrestricted preference domains}

Before considering simultaneous restrictions on the preference and agenda domains, it is useful to consider a number of social choice problems in which the preference domain is unrestricted. Unrestricted preference domains do not arise naturally in economic problems, but are often natural in voting 
problems. In Section 13.1, we discuss a finite-alternative generalization of the choice-theoretic version of Arrow's Theorem due to Grether and Plott (1982). In Section 13.2, we present a theorem due to Dutta, Jackson, and Le Breton (2001) on the impossibility of constructing a Paretian voting procedure that is immune to strategic entry decisions on the part of the candidates. As recently shown by Ehlers and Weymark (2003), the Grether-Plott Theorem can be used to help prove Dutta, Jackson, and Le Breton's candidate stability theorem. In Section 13.3, we discuss the work of Gibbard, Hylland, and Weymark (1987) on agenda domains in which one or more alternatives are in every agenda. Such agenda domains capture some of the institutional features of committee and legislative decision-making.

Some of the agenda domains that we consider in this section have the property that some, or all, of the binary agendas are not admissible. This is the usual framework employed in the theory of nonbinary social choice theory. The literature on nonbinary social choice when the preference domain is unrestricted is quite extensive, but, as noted above, it has limited relevance for economic problems. See Deb (2004) for a survey of this literature.

\subsection{The Grether-Plott Theorem}

The proof of Arrow's Theorem presented in Section 12.2 makes essential use of the assumption that all binary agendas are in the domain. Grether and Plott (1982) noted that economic and political problems typically have agenda domains in which this assumption is violated. To overcome this problem, they relaxed the complete finite agenda domain assumption. Assuming that the universal set of alternatives $X$ is finite, their agenda domain is the set of all agendas containing at least $k$ alternatives, where $k<|X|$.

$k$-Set Feasibility (KSF). There exists a finite positive integer $k<|X|$ such that $A \in \mathcal{A}$ if and only if $|A| \geq k$.

The value of $k$ must be strictly less than the number of alternatives in order for ACA to play any role. This domain is appropriate if only the "large" agendas are feasible, with the parameter $k$ being used to identify when an agenda is "large".

For a finite universal set of alternatives, the Grether-Plott Theorem shows that the Arrow axioms are inconsistent when the preference domain is unre- 
stricted and KSF is satisfied. ${ }^{87}$

Theorem 20. If $3 \leq|X|<\infty$, there is no social choice correspondence with an unrestricted preference domain that satisfies KSF, ACA, IIF, WP, and ND.

When KSF is satisfied, the agenda domain is closed under finite unions. Hence, by Hansson's Theorem (Theorem 18), ACA implies that the social choice correspondence can be rationalized by a social welfare function. However, if $k>2$, this rationalization is typically not unique. For example, suppose that $X=\{w, x, y, z\}, k=3$, and $C(X, \mathbf{R})=\{w, x\}$. For this profile, the choice set for any of the three-alternative agendas is completely determined by ACA. However, the ranking of $y$ and $z$ is arbitrary in any ordering that rationalizes these choices. Because of the possible non-uniqueness of the rationalizing social welfare function, the strategy used to prove Arrow's Theorem in Section 12.2 cannot be used to prove Theorem 20.

Grether and Plott's proof establishes that if a social choice correspondence satisfies all of the assumptions of Theorem 20 with $k \geq 3$, then it is is possible to construct another social choice correspondence with the same preference domain that satisfies $(k-1)$-Set Feasibility and all of the Arrow axioms. An induction argument then shows that the Arrow axioms are consistent when all nonempty finite feasible sets are feasible, which is impossible. Note that if $k$ is 1 or 2 , Theorem 20 is simply a finite version of Arrow's Theorem.

It is instructive to consider how Grether and Plott constructed the social choice correspondence used in their proof. For $h=k-1, k$, let $\mathcal{A}^{h}=\{A \in$ $\mathcal{A}|| A \mid \geq h\}$. Suppose that $3 \leq k<|X|$ and that $C^{k}: \mathcal{A}^{k} \times \mathcal{R}^{n} \rightarrow X$ satisfies the Arrow axioms. For all $A \in \mathcal{A}^{k-1}, z \in X \backslash A$, and $\mathbf{R} \in \mathcal{R}^{n}$, let $\overline{\mathbf{R}}(A, z, \mathbf{R})$ denote the unique profile in $\mathcal{R}^{n}$ for which, for all $i \in N$,

(i) $\forall x, y \in A, x R_{i} y \leftrightarrow x \bar{R}_{i}(A, z, \mathbf{R}) y$,

(ii) $\forall x \in A, x \bar{R}_{i}(A, z, \mathbf{R}) z$ and $\neg\left[z \bar{R}_{i}(A, z, \mathbf{R}) x\right]$,

(iii) $\forall x \notin A \cup\{z\}, z \bar{R}_{i}(A, z, \mathbf{R}) x$ and $\neg\left[x \bar{R}_{i}(A, z, \mathbf{R}) z\right]$,

(iv) $\forall x, y \notin A \cup\{z\}, x R_{i} y \leftrightarrow x \bar{R}_{i}(A, z, \mathbf{R}) y$.

In other words, for each individual $i$, the alternatives in $A$ have been moved to the top and the alternatives not in $A \cup\{z\}$ have been moved to the bottom of $i$ 's preference ordering, preserving the original rankings in each of these

\footnotetext{
${ }^{87}$ The assumption that there are only a finite number of alternatives limits the relevance of the Grether-Plott Theorem for economic problems with divisible goods.
} 
groups. The alternatives in $A$ are all preferred to $z$, which in turn is preferred to all of the remaining alternatives.

The social choice correspondence $C^{k-1}: \mathcal{A}^{k-1} \times \mathcal{R}^{n} \rightarrow X$ is defined by setting

$$
C^{k-1}(A, \mathbf{R})=C^{k}(A, \mathbf{R}), \forall A \in \mathcal{A}^{k}, \forall \mathbf{R} \in \mathcal{R}^{n}
$$

and

$$
C^{k-1}(A, \mathbf{R})=\bigcup_{z \notin A} C^{k}(A \cup\{z\}, \bar{R}(A, z, \mathbf{R})), \forall A \in \mathcal{A}^{k-1} \backslash \mathcal{A}^{k}, \forall \mathbf{R} \in \mathcal{R}^{n} .
$$

Because $C^{k}$ satisfies WP, $\emptyset \neq C^{k}(A \cup\{z\}, z, \bar{R}(A, z, \mathbf{R})) \subseteq A$, and thus $C^{k-1}$ is a well-defined social choice correspondence.

Because $C^{k}$ and $C^{k-1}$ agree on agendas in $\mathcal{A}^{k}$ and $C^{k}$ satisfies ND, so does $C^{k-1}$. When $\mathbf{R}^{1}$ and $\mathbf{R}^{2}$ coincide on $A \in \mathcal{A}^{k-1}, \bar{R}\left(A, z, \mathbf{R}^{1}\right)$ and $\bar{R}\left(A, z, \mathbf{R}^{2}\right)$ coincide on $A$ as well. It thus follows from the assumption that $C^{k}$ satisfies IIF that $C^{k-1}$ does too. Because $C^{k}$ satisfies WP, so does $C^{k-1}$. The proof that $C^{k-1}$ satisfies ACA is quite lengthy. See Grether and Plott (1982) for the details.

In Grether and Plott's proof, it is necessary to move a set of alternatives to the top of each person's preferences, while preserving their relative rankings. The assumption that the preference domain is unrestricted ensures that this is possible. However, in economic problems with divisible goods, preferences are typically assumed to be continuous. With continuous preferences, this kind of construction is not possible, which suggests that the proof strategy used by Grether and Plott is not likely to be applicable to problems with divisible goods and continuous preferences.

\subsection{Strategic candidacy}

By interpreting $X$ as a finite set of potential candidates in an election and $N$ as the set of voters, a social choice correspondence can be used to model elections in which the number of candidates is endogenous. Dutta, Jackson, and Le Breton (2001) have used this framework to investigate voting procedures in which the outcome of an election is unaffected by the decision not to stand for office of a candidate who would not win if he or she entered the election. We consider the case in which no candidate is also a voter.

An agenda is now interpreted as the set of candidates who contest the election. Dutta, Jackson, and Le Breton assumed that there are at least 
three potential candidates and that any subset of potential candidates could be on the ballot. Thus, the social choice correspondence has a complete finite agenda domain. Voters submit their rankings of the candidates, with no ties permitted. The preference domain is the unrestricted linear preference domain introduced in Section 2. ${ }^{88}$ Only one candidate can be elected, so the social choice correspondence is in fact a social choice function.

Single-Valuedness (SV). For all $A \in \mathcal{A}$ and all $\mathbf{R} \in \mathcal{D}, C(A, \mathbf{R})$ is a singleton.

The voting procedure described by the social choice correspondence is assumed to be invulnerable to the withdrawal of a candidate who would lose if all potential candidates stood for office.

Candidate Stability (CS). For all $x \in X$ and all $\mathbf{R} \in \mathcal{D}$, if $x \notin C(X, \mathbf{R})$, then $C(X, \mathbf{R})=C(X \backslash\{x\}, \mathbf{R})$.

$\mathrm{CS}$ is a relatively weak way of requiring a voting procedure to be immune to the strategic entry decisions of the candidates because it only applies when all of the other potential candidates enter the election. ${ }^{89}$

Unanimity requires a candidate to be elected who is ranked first on the ballot by all of the voters.

Unanimity (UNAN). For all $A \in \mathcal{A}, x \in A$, and $\mathbf{R} \in \mathcal{D}$, if for all $i \in N, x P_{i} y$ for all $y \in A \backslash\{x\}$, then $C(A, \mathbf{R})=\{x\}$.

Dutta, Jackson, and Le Breton used a strengthened form of the Arrovian nondictatorship condition. An individual is a dictator for large elections if

\footnotetext{
${ }^{88}$ The voting rules used by Dutta, Jackson, and Le Breton (2001) choose a candidate from an agenda as a function of the preferences of both voters and candidates. A candidate is assumed to rank him- or herself first, but otherwise can have any linear order. Dutta, Jackson, and Le Breton assumed that the election outcome only depends on the voters' preferences, which permits us to simplify the statement of the problem as we have done here when candidates cannot vote.

${ }^{89}$ The candidate stability axiom used here is what Dutta, Jackson, and Le Breton have called Strong Candidate Stability. They also considered a second candidate stability axiom that requires each candidate to prefer the outcome when all candidates are on the ballot to the outcome that would obtain if he or she withdrew from the election. As Dutta, Jackson, and Le Breton have shown, when the sets of voters and candidates are disjoint, these two candidate stability axioms are equivalent, given their other assumptions. When candidates and voters have individuals in common, this equivalence no longer holds.
} 
this individual's most-preferred candidate is elected when at least $|X|-1$ candidates run for office. Formally, an individual $d \in N$ is a dictator for large elections for the social choice correspondence $C$ if for all $A \in \mathcal{A}$ with $|A| \geq|X|-1$ and all $\mathbf{R} \in \mathcal{D}, C(A, \mathbf{R})=\{x\}$ whenever $x \in A$ and $x P_{d} y$ for all $y \in A \backslash\{x\}$.

Strong Nondictatorship (SND). There is no dictator for large elections.

Dutta, Jackson, and Le Breton (2001) have shown that no voting procedure can satisfy IIF in addition to the assumptions described above.

Theorem 21. If $3 \leq|X|<\infty$, there is no social choice correspondence with a complete finite agenda domain and an unrestricted linear preference domain that satisfies SV, IIF, UNAN, CS, and SND. ${ }^{90}$

Both Arrow's Theorem and the Grether-Plott Theorem are valid for the preference domain $\mathcal{L}^{n}$. However, because UNAN is, by itself, a much weaker condition than WP, the Dutta-Jackson-Le Breton Theorem does not follow directly from either of these results. For this reason, they do not exploit either of these theorems in proving Theorem 21.

Ehlers and Weymark (2003) have shown that the Grether-Plott Theorem can be used to help prove Theorem 21 . Their proof strategy begins by assuming that the social choice correspondence $C$ satisfies all of the assumptions of Theorem 21 except SND. By then restricting attention to the agendas with at least $|X|-1$ candidates, the resulting social choice correspondence on the subdomain inherits all of the assumed properties of $C$. On this domain, $\mathrm{CS}$ is equivalent to ACA for single-valued social choice correspondences. In addition, the new agenda domain satisfies $k$-Set Feasibility for $k=|X|-1$. On the subdomain, the social choice correspondence also satisfies WP, and, hence, by the Grether-Plott Theorem, it must be dictatorial, which, in terms of the original domain, is equivalent to the existence of a dictator for large elections. The proof that $C$ satisfies WP on the subdomain is somewhat lengthy. See Ehlers and Weymark (2003) for the details.

\footnotetext{
${ }^{90}$ When candidates are permitted to vote, Dutta, Jackson, and Le Breton (2001) have shown that if CS is combined with some other reasonable properties of voting procedures, then the distribution of power among voters must be quite unequal.
} 


\subsection{Fixed feasible alternatives}

In Gibbard, Hylland, and Weymark (1987), there is a finite set of alternatives and it is assumed that there is some alternative or set of alternatives that is in every feasible agenda. Examples with a single fixed feasible alternative include (i) allocation problems with fixed endowments in which preserving the initial distribution is always an option and (ii) bargaining problems in which there is a prespecified alternative (the threat point) that is chosen if no agreement is reached on the choice of another alternative. In committee or legislative decision-making, the rules of order may specify that the motion and the status quo are always under consideration, with various possible amendments generating three-alternative agendas. In this example, there are two fixed alternatives in every admissible agenda.

For a nonempty subset $\bar{A}$ of $X$, the agenda domain is $\bar{A}$-restricted if $\bar{A}$ is in every feasible agenda.

$\bar{A}$-Restricted Agenda Domain. $A \in \mathcal{A}$ implies $\bar{A} \subseteq A$.

This domain restriction does not require that every superset of $\bar{A}$ is a feasible agenda. An agenda domain with this property is called complete.

Complete $\bar{A}$-Restricted Agenda Domain. $A \in \mathcal{A}$ if and only if $\bar{A} \subseteq A$.

Gibbard, Hylland, and Weymark (1987) have shown that for any $\bar{x} \in X$, Arrow's axioms, with WP strengthened to SP, are consistent when the agenda domain is $\bar{x}$-restricted and the preference domain is unrestricted. ${ }^{91}$

Theorem 22. If $2 \leq|X|<\infty$, for any $\bar{x} \in X$, there exist social choice correspondences with an $\{\bar{x}\}$-restricted agenda domain and an unrestricted preference domain that satisfy ACA, IIF, SP, and ND.

The proof of this theorem is constructive. The social choice correspondence Gibbard, Hylland, and Weymark used to establish Theorem 22 is presented in Example 23.

Example 23. Fix $\bar{x} \in X$ and suppose that $\mathcal{A}$ is $\{\bar{x}\}$-restricted. For all $x \in X$ and $\mathbf{R} \in \mathcal{R}^{n}$, let $N^{+}(x, \mathbf{R})=\left|\left\{i \in N \mid x P_{i} \bar{x}\right\}\right|, N^{-}(x, \mathbf{R})=\mid\{i \in N \mid$

\footnotetext{
${ }^{91}$ The Arrow axioms are also consistent for any $\bar{A}$-restricted agenda domain when the preference domain is unrestricted.
} 
$\left.\bar{x} P_{i} x\right\} \mid$, and $N(x, \mathbf{R})=N^{+}(x, \mathbf{R})-N^{-}(x, \mathbf{R})$. If every individual assigns one point to each alternative preferred to $\bar{x}$, zero points to each alternative indifferent to $\bar{x}$, and negative one point to every other alternative, then $N(x, \mathbf{R})$ is the total number of points assigned to $x$ in the profile $\mathbf{R}$.

For all $A \in \mathcal{A}$ and $\mathbf{R} \in \mathcal{R}^{n}$, let

$$
\begin{gathered}
C^{R}(A, \mathbf{R})=\{x \in A \mid \forall y \in A, N(x, \mathbf{R}) \geq N(y, \mathbf{R})\}, \\
C^{0}(A, \mathbf{R})=\left\{x \in C^{R}(A, \mathbf{R}) \mid \forall y \in C^{R}(A, \mathbf{R}), N^{-}(x, \mathbf{R}) \leq N^{-}(y, \mathbf{R})\right\},
\end{gathered}
$$

and, for all $i \in N$,

$$
C^{i}(A, \mathbf{R})=\left\{x \in C^{i-1}(A, \mathbf{R}) \mid \forall y \in C^{i-1}(A, \mathbf{R}), x R_{i} y\right\} .
$$

The social choice correspondence $C^{n}$ satisfies all of the assumptions of Theorem 22 .

$C^{n}$ satisfies ACA because it is rationalized by a lexicographic social welfare function. For a given profile, the alternatives are first ranked by the number of points received. Alternatives that are tied at this stage are then ranked in reverse order of the number of negative votes received. Finally, a serial dictatorial ordering is used as a secondary tie-breaking rule.

Richelson (1984) considered the social choice correspondence $C^{R}$. The use of $C^{R}$ and the first tie-breaking rule ensure that $C^{n}$ is nondictatorial. ${ }^{92} C^{R}$ violates WP because if everyone prefers $x$ to $y$ to $\bar{x}$ and the agenda contains $\{x, y, \bar{x}\}$, then $x$ and $y$ receive the same number of points, even though they are Pareto ranked. The use of a serial dictatorship as a tie-breaking rule ensures that $C^{n}$ satisfies SP. Because $C^{n}(A, \mathbf{R})$ is determined using only information about the individual preferences on $A, C^{n}$ satisfies IIF.

Gibbard, Hylland, and Weymark have provided other examples of social choice correspondences that satisfy the Arrow axioms when the agenda domain is $\bar{A}$-restricted. Example 24 requires $\bar{A}$ to include two alternatives.

Example 24. Fix $\bar{x}, \bar{y} \in X$ and suppose that $\mathcal{A}$ is $\{\bar{x}, \bar{y}\}$-restricted. There are $3^{n}$ possible preference profiles restricted to $\{\bar{x}, \bar{y}\}$, which we number arbitrarily from 1 to $3^{n}$. Let $\mathcal{D}^{j}$ denote the set of all profiles in $\mathcal{R}^{n}$ that

\footnotetext{
${ }^{92}$ If the first tie-breaking rule is not included, person one is a dictator when $n=2$ or $|A| \leq 2$ for all $A \in \mathcal{A}$.
} 
coincide with the $j$ th of these restricted preference profiles on $\{\bar{x}, \bar{y}\}$. Let $d:\left\{1, \ldots, 3^{n}\right\} \rightarrow N$ be a surjective function. The social choice correspondence $C: \mathcal{A} \times \mathcal{R}^{n} \rightarrow X$ is defined by setting $C(A, \mathbf{R})=B\left(A, R_{d(j)}\right)$ for all $A \in \mathcal{A}$, all $j \in\left\{1, \ldots, 3^{n}\right\}$, and all $\mathbf{R} \in \mathcal{D}^{j}$. In other words, restricted to profiles in $\mathcal{D}^{j}$, individual $d(j)$ is a strong dictator.

Because, for each profile, the choice sets are determined by maximizing some individual's preference ordering, both ACA and WP satisfied. ${ }^{93}$ Because the agenda domain is $\{\bar{x}, \bar{y}\}$-restricted, if two profiles coincide on a feasible agenda, they coincide on $\{\bar{x}, \bar{y}\}$. As a consequence, the same dictator is used for both profiles, so IIF is satisfied. Each individual is a dictator for some subset of the profiles, so $C$ is nondictatorial.

Restricted to preference profiles in $\mathcal{L}^{n}$, the lexicographic social welfare function used to construct the social choice correspondence in Example 23 is an example of what Campbell and Kelly (2000) have called a three-layer gateau rule. With a three-layer gateau rule, for each profile $\mathbf{R}$ in the domain $\mathcal{D}$, the alternatives are partitioned into three groups, $T(\mathbf{R}), M(\mathbf{R})$, and $B(\mathbf{R})$, with all the alternatives in $T(\mathbf{R})$ socially ranked above the alternatives in $M(\mathbf{R})$, which, in turn, are all socially ranked above the alternatives in $B(\mathbf{R})$ (the three layers of a cake). More precisely, a social welfare function $F: \mathcal{D} \rightarrow \mathcal{R}$ is a three-layer gateau rule if there exists an $\bar{x} \in X$ and two families $\mathbf{N}_{1}$ and $\mathbf{N}_{2}$ of subsets of $N$ such that,

(i) $I \cap J \neq \emptyset$ if $I \in \mathbf{N}_{1}$ and $J \in \mathbf{N}_{2}$,

(ii) $I \subseteq N$ and $I \notin \mathbf{N}_{1} \rightarrow N \backslash I \in \mathbf{N}_{2}$,

(iii) $\forall \mathbf{R} \in \mathcal{D}, T(\mathbf{R})=\left\{x \in X \mid \exists I \in \mathbf{N}_{1}\right.$ such that $\left.\forall i \in I, x P_{i} \bar{x}\right\}$,

(iv) $\forall \mathbf{R} \in \mathcal{D}, B(\mathbf{R})=\left\{x \in X \mid \exists J \in \mathbf{N}_{2}\right.$ such that $\left.\forall i \in J, \bar{x} P_{i} x\right\}$,

(v) $\forall \mathbf{R} \in \mathcal{D}, M(\mathbf{R})=X \backslash[T(\mathbf{R}) \cup B(\mathbf{R})]$,

where $T(\mathbf{R}), M(\mathbf{R})$, and $B(\mathbf{R})$ are socially ranked as described above.

The distinguished alternative $\bar{x}$ is in $M(\mathbf{R})$. It is easy to show that if $\mathbf{R} \in \mathcal{L}^{n}$, then $\bar{x}$ is the only alternative in $M(\mathbf{R})$. Any group of individuals $I \in \mathbf{N}_{1}$ forces $x$ to be in the top layer if everyone in this group prefers $x$ to $\bar{x}$ and any group of individuals $J \in \mathbf{N}_{2}$ forces $x$ to be in the bottom layer if everyone in this group prefers $\bar{x}$ to $x$. Condition (i) ensures that no alternative is put in more than one layer. Restricted to preference profiles in $\mathcal{L}^{n}$, the social welfare function used to construct Example 23 is a three-layer gateau rule with the following specification of the families $\mathbf{N}_{1}$ and $\mathbf{N}_{2}$ : (i)

\footnotetext{
${ }^{93}$ If a serial dictatorship is used for each $\mathcal{D}^{j}$ to determine the choice sets, SP is satisfied.
} 
for odd $n, \mathbf{N}_{1}=\mathbf{N}_{2}=\{I \subseteq N|| I \mid>n / 2\}$ and (ii) for even $n, \mathbf{N}_{1}=\{I \subseteq$ $N|| I \mid>n / 2\}$ and $\mathbf{N}_{2}=\{I \subseteq N|| I \mid \geq n / 2\}$.

Three-layer gateau rules are social welfare functions that satisfy a weaker form of IIA in which the social ranking of a pair of alternatives only depends on the individual rankings of this pair and some prespecified alternative $\bar{x}$.

Independence of Irrelevant Alternatives Relative to $\bar{x}$ (IIA $\bar{x}$ ). For all $x, y \in X$ and all $\mathbf{R}^{1}, \mathbf{R}^{2} \in \mathcal{D}$, if $\mathbf{R}^{1}$ and $\mathbf{R}^{2}$ coincide on $\{x, y, \bar{x}\}$, then $R^{1}$ and $R^{2}$ coincide on $\{x, y\}$.

Campbell and Kelly (2000) have shown that if there are at least four alternatives and the preference domain is $\mathcal{L}^{n}$, then any social welfare function that satisfies this independence condition and is weakly Paretian must be a three-layer gateau rule. ${ }^{94}$

Theorem 23. If $|X| \geq 4$, for any $\bar{x} \in X$, if a social welfare function with an unrestricted linear preference domain satisfies IIA $\bar{x}$ and $\mathrm{WP}$, then it is a three-layer gateau rule.

Consider a social choice correspondence $C$ with a complete $\bar{x}$-restricted agenda domain and the preference domain $\mathcal{L}^{n}$. This agenda domain is closed under finite unions, so, by Hansson's Theorem, $C$ can be rationalized by a social welfare function $F$ whose domain is $\mathcal{L}^{n}$. Using this observation, it might seem that the Campbell-Kelly Theorem can be used to characterize the social choice correspondences that satisfy IIF and WP on such domains. However, this is not the case. As we have already noted, when not all the binary agendas are feasible, ACA does not guarantee that there is a unique social welfare function rationalizing the social choice correspondence. This is the case here. For example, consider a profile in which everyone prefers $\bar{x}$ to both $x$ and $y$. WP implies that $\bar{x}$ is chosen out of $\{x, y, \bar{x}\}$, so the social ranking of $x$ and $y$ is arbitrary. As a consequence, it does not follow from the assumption that $C$ satisfies IIF that $F$ satisfies IIA $\bar{x}$. The nonuniqueness of the rationalizing social welfare function makes it a challenging problem to characterize all of the social choice correspondences that satisfy the Arrow axioms on a complete $\bar{x}$-restricted agenda domain.

Examples 23 and 24 both exhibit dictatorial features. In Example 23, a serial dictatorship is used as a tie-breaking rule, while in Example 24, the

\footnotetext{
${ }^{94}$ Yanovskaya (1994) has investigated the implications of IIA $\bar{x}$ in a framework in which social preferences are merely required to be reflexive and transitive.
} 
preference domain has been partitioned into subsets on which someone is a dictator. For any complete $\bar{A}$-restricted agenda domain, Gibbard, Hylland, and Weymark (1987) have shown that if the preference domain is unrestricted and there are at least three alternatives in $X$ not in $\bar{A}$, then it is not possible to satisfy the Arrow axioms with ND strengthened to ANON. Thus, it is inevitable that any social choice correspondence satisfying the assumptions of Theorem 22 cannot avoid all vestiges of dictatorship on such domains.

Theorem 24. For any nonempty $\bar{A} \subseteq X$, if $|\bar{A}|+3 \leq|X|<\infty$, there is no social choice correspondence with a complete $\bar{A}$-restricted agenda domain and an unrestricted preference domain that satisfies ACA, IIF, WP, and ANON.

Proof. Let $C: \mathcal{A} \times \mathcal{R}^{n} \rightarrow X$ satisfy all of the assumptions of the theorem except ANON. Let $\mathcal{D}_{\bar{A}}$ be a subdomain of profiles in which (i) everyone ranks every alternative not in $\bar{A}$ above the alternatives in $\bar{A}$ and (ii) the individual rankings of the alternatives in $\bar{A}$ agree with some fixed set of individual orderings (e.g., have everyone regard these alternatives as being indifferent). Let $Y=X \backslash \bar{A}$ and $\left.\mathbf{R}\right|_{Y},\left.\mathcal{D}_{\bar{A}}\right|_{Y},\left.A\right|_{Y}$, and $\left.\mathcal{A}\right|_{Y}$ denote the restrictions of $\mathbf{R}$, $\mathcal{D}_{\bar{A}}, A$, and $\mathcal{A}$, respectively, to $Y$. Define the social choice correspondence $C^{*}:\left.\mathcal{A}\right|_{Y} \times\left.\mathcal{D}_{\bar{A}}\right|_{Y} \rightarrow Y$ by setting $C^{*}\left(A_{Y},\left.\mathbf{R}\right|_{Y}\right)=C(A, \mathbf{R})$ for all $A \in \mathcal{A}$ and all $\mathbf{R} \in \mathcal{D}_{\bar{A}}$. Because $C$ satisfies WP, the alternatives in $\bar{A}$ are never chosen from any agenda when the profile is in $\mathcal{D}_{\bar{A}}$. Hence, $C^{*}$ is well-defined. Because $\mathcal{A}$ is a complete $\bar{A}$-restricted agenda domain, $\left.\mathcal{A}\right|_{Y}$ is a complete finite agenda domain for $Y$. Further, $C^{*}$ has an unrestricted preference domain. It is straightforward to confirm that $C^{*}$ satisfies ACA, IIF, and WP. Because $|Y| \geq 3$, it follows from Arrow's Theorem that $C^{*}$ is dictatorial, which implies that $C$ does not satisfy ANON.

The conclusions to be drawn from the results discussed in this section are essentially negative - either the axioms are inconsistent or any social choice correspondence satisfying the axioms necessarily exhibits some dictatorial features. This suggests that restricting the agenda domain while keeping the preference domain unrestricted is not a very promising way of avoiding Arrovian impossibilities. 


\section{Single-peaked preferences and interval agenda do- mains}

We now turn to restricted preference domains. In this section, we suppose that the set of alternatives $X$ is a compact interval $[\underline{x}, \bar{x}]$ of $\mathbb{R}$ with $\underline{x}<\bar{x}$. The agenda domain consists of all the nonempty closed intervals of $X$, which we denote by $\mathcal{A}_{I}$. For a preference domain, we consider the unrestricted single-peaked preference domain $\mathcal{S}_{P}^{n}$ introduced in Section 3. With these domain assumptions, the Arrow axioms with ND strengthened to ANON are consistent. We describe one class of social choice functions that satisfy all of these axioms, the class of generalized median social choice functions introduced in Moulin (1980), and we present an axiomatization of this class due to Moulin (1984).

We refer to the agenda domain considered in this section as a complete closed interval agenda domain.

Complete Closed Interval Agenda Domain. $\mathcal{A}=\mathcal{A}_{I}$.

Note that $\mathcal{A}_{I}$ is the set of all nonempty, compact, convex subsets of $X$.

For all $R \in \mathcal{S}_{P}$, let $\pi(R)$ denote the peak of $R$. Consider any $A=[a, b] \in$ $\mathcal{A}_{I}$ and any $R \in \mathcal{S}_{P}$. The restriction of $R$ to $A$ is single-peaked with peak at $\operatorname{Pr}_{A} \pi(R)$, the projection of $\pi(R)$ on $A$. Thus, $B(A, R)=\pi(R)$ if $\pi(R) \in A$, $B(A, R)=a$ if $\pi(R)<a$, and $B(A, R)=b$ if $\pi(R)>b$.

Following Moulin (1984), we assume that choice is single-valued. One interpretation of our problem is that we are to locate a public facility on a street. Each individual has a preferred location, with preference declining monotonically from this ideal location. Not all locations may be feasible, but the ones that are form a closed interval.

Moulin (1984) required the alternative chosen to vary continuously with the endpoints of the feasible set for a given preference profile. This continuity axiom presupposes that the social choice correspondence $C$ is single-valued.

Interval Continuity (IC). For all $\mathbf{R} \in \mathcal{D}$, the function $C(\cdot, \mathbf{R})$ is continuous at $[a, b]$ with respect to $a$ and $b$ for all $\underline{x} \leq a<b \leq \bar{x}$.

The agenda domain $\mathcal{A}_{I}$ is not closed under finite unions, so we cannot appeal to Hansson's Theorem (Theorem 18) to conclude that ACA is equivalent to the existence of a social welfare function that rationalizes the social choice 
correspondence $C: \mathcal{A}_{I} \times \mathcal{S}_{P}^{n} \rightarrow X$. Nevertheless, Moulin (1984) has shown that this equivalence holds if $C$ is single-valued and satisfies IC. Further, for each profile, the social preference that rationalizes the choice in each agenda can be chosen to be single-peaked.

Lemma 5. If the social choice correspondence $C: \mathcal{A}_{I} \times \mathcal{S}_{P}^{n} \rightarrow X$ satisfies $\mathrm{SV}$ and IC, then $C$ can be rationalized by a social welfare function $F: \mathcal{S}_{P}^{n} \rightarrow \mathcal{S}_{P}$ if and only if $C$ satisfies ACA.

Proof. Suppose that $C$ satisfies ACA. Consider any $\mathbf{R} \in \mathcal{S}_{P}^{n}$. Let $\beta=$ $C(X, \mathbf{R})$ and let $R^{\beta} \in \mathcal{S}_{P}$ have peak $\beta$. Now consider any $[a, b] \in \mathcal{A}_{I}$. We want to show that $B\left([a, b], R^{\beta}\right)=C([a, b], \mathbf{R})$. If $\beta \in A$, this follows immediately from ACA. If $\beta \notin[a, b]$, we may without loss of generality assume that $\beta<a$, which implies that $B\left([a, b], R^{\beta}\right)=a$. Suppose that $C([a, b], \mathbf{R})=c>a$. By ACA, $C([\underline{x}, b], \mathbf{R})=\beta$. Because $C([\underline{x}, b], \mathbf{R})=\beta$ and $C([a, b], \mathbf{R})=c$, by $\mathrm{IC}$, there must exist an $a^{\prime} \in(\underline{x}, a)$ such that $C\left(\left[a^{\prime}, b\right], \mathbf{R}\right)=a$. ACA then implies that $C([a, b], \mathbf{R})=a$, a contradiction. Hence, $B\left([a, b], R^{\beta}\right)=C([a, b], \mathbf{R})$ in this case as well.

The reverse implication is straightforward to verify. ${ }^{95}$

Moulin (1980) introduced the following class of generalized median social choice functions for the agenda domain in which $X$ is the only feasible set. He extended his definition to the domain $\mathcal{A}_{I}$ in Moulin (1984).

Generalized Median Social Choice Function. A social choice function $C: \mathcal{A}_{I} \times$ $\mathcal{S}_{P}^{n} \rightarrow X$ is a generalized median social welfare function if there exists a profile $\mathbf{R}^{P}=\left(R_{n+1}^{P}, \ldots, R_{2 n-1}^{P}\right) \in \mathcal{S}_{P}^{n-1}$ such that for all $A \in \mathcal{A}_{I}$ and all $\mathbf{R} \in \mathcal{S}_{P}^{n}$,

$$
C(A, \mathbf{R})=\operatorname{Pr}_{A} \operatorname{median}\left\{\pi\left(R_{1}\right), \ldots, \pi\left(R_{n}\right), \pi\left(R_{n+1}^{P}\right), \ldots, \pi\left(R_{2 n-1}^{P}\right)\right\} .{ }^{96}
$$

As in the construction of a generalized median social welfare function in Example 1, fixed single-peaked preferences for $n-1$ phantom individuals are specified. For each profile $\mathbf{R} \in \mathcal{S}_{P}^{n}$, the median of the peaks of the $2 n-1$ real and phantom individuals is determined. For any agenda $A \in \mathcal{A}_{I}$, the choice

\footnotetext{
${ }^{95}$ Our proof of this lemma is based on the proof of Lemma 2.1 in Ehlers (2001). Note that the assumption that individual preferences are single-peaked is not used in the proof.

${ }^{96}$ For each $j \in\{n+1, \ldots, 2 n-1\}$, it is only necessary to specify the peak, and not the complete preference ordering. Generalized median social choice functions are called generalized Condorcet-winner social choice functions in Moulin (1984).
} 
set $C(A, \mathbf{R})$ is the projection of this median peak to $A$. Equivalently, $C(A, \mathbf{R})$ is the best alternative in $A$ for the the individual (either real or phantom) with the median peak. It is straightforward to verify that $C(A, \mathbf{R})$ can also be determined by first projecting all $2 n-1$ peaks onto $A$ and then computing the median of these projected peaks.

Recall from Section 3 that the binary relation $\leq$ (resp. $\geq$ ) on $X$ declares $x$ to be weakly preferred to $y$ if and only if $x \leq y$ (resp. $x \geq y$ ). If $n$ is odd, half of the phantoms have the preference $\leq$, and the other half have the preference $\geq$, then the phantom individuals are irrelevant and $C(A, \mathbf{R})$ is obtained by maximizing the preference of the (real) individual with the median peak. If there are $n-k$ phantoms with preference $\leq$ and $k-1$ with preference $\geq, C(A, \mathbf{R})$ maximizes the preference of the individual with the $k$ th smallest peak.

Suppose the $C$ is a generalized median social choice function. Because the number of phantom individuals is less than the number of real individuals, the median peak in $\left(\mathbf{R}, \mathbf{R}^{P}\right)$ must lie in the interval defined by the smallest and largest peaks in $\mathbf{R}$. As a consequence, $C$ satisfies SP. ${ }^{97}$ For a fixed profile, the choices from different agendas are determined by maximizing the same preference, so $C$ satisfies ACA. If the profiles $\mathbf{R}^{1}$ and $\mathbf{R}^{2}$ coincide on the agenda $A$, then the projections of the individual peaks coincide as well. Hence, $C$ satisfies IIF. It is clear that a generalized median social choice function also satisfies ANON and IC. Moulin (1984) has shown that these five axioms characterize the class of generalized median social choice functions. To facilitate the comparison of this result with the other theorems in Part II, we state Moulin's theorem as a theorem about social choice correspondences.

Theorem 25. For any $X=[\underline{x}, \bar{x}] \subset \mathbb{R}$ with $\underline{x}<\bar{x}$, if a social choice correspondence has a complete closed interval agenda domain and an unrestricted single-peaked preference domain, then it satisfies SV, ACA, IIF, SP, ANON, and IC if and only if it is a generalized median social choice function.

By Lemma 5, SV, ACA, and IC imply that a social choice correspondence $C: \mathcal{A}_{I} \times \mathcal{S}_{P}^{n} \rightarrow X$ can be rationalized by a social welfare function $F: \mathcal{S}_{P}^{n} \rightarrow$ $\mathcal{S}_{P}$. For $k=1, \ldots, n-1$, let $\mathbf{R}^{k}$ be the profile in which $R_{i}^{k}=\leq$ for $i=$ $1, \ldots, k$ and $R_{i}^{k}=\geq$ for $i=k+1, \ldots, n$ and let $\beta_{k}=\pi\left(F\left(\mathbf{R}^{k}\right)\right)$. The sufficiency part of the proof of Theorem 25 involves showing that $C$ is the generalized median social choice function defined by the profile of phantom

\footnotetext{
${ }^{97} \mathrm{WP}$ and SP are equivalent for the domain $\mathcal{A}_{I} \times \mathcal{S}_{P}^{n}$.
} 
preferences $\overline{\mathbf{R}}^{P}=\left(F\left(\mathbf{R}^{1}\right), \ldots, F\left(\mathbf{R}^{n-1}\right)\right)$. To do this, it is sufficient to show that for all $\mathbf{R} \in \mathcal{S}_{P}^{n}, \pi(F(\mathbf{R}))=\operatorname{median}\left\{\pi\left(R_{1}\right), \ldots, \pi\left(R_{n}\right), \beta_{1}, \ldots, \beta_{n-1}\right\}$. See Moulin (1984) for the details of the proof.

Moulin (1984) has also considered the domains of single-plateaued and quasiconcave preferences. A preference $R$ on $X=[\underline{x}, \bar{x}]$ is single-plateaued if there exist $\beta_{1}, \beta_{2} \in X$ (not necessarily distinct) such that (i) $x P y$ whenever $\beta_{1} \geq x>y$ or $\beta_{2} \leq x<y$ and (ii) $x I y$ whenever $x, y \in\left[\beta_{1}, \beta_{2}\right]$. A preference $R$ on $X=[\underline{x}, \bar{x}]$ is quasiconcave if there exists a $\beta \in X$ such that (i) $x R y$ whenever $\beta \geq x>y$ or $\beta \leq x<y .{ }^{98}$ A single-peaked preference is singleplateaued and a single-plateaued preference is quasiconcave. Moulin has shown that a version of Theorem 25 holds for single-plateaued preferences and that his axioms are incompatible when the preference domain includes all profiles of quasiconcave preferences.

For the domain of Theorem 25, Ehlers (2001) has considered the problem of choosing exactly $m$ alternatives from each agenda, where $m<n$ (so that it is not possible to always pick everyone's preferred alternative). Thus, a social alternative consists of $m$ (not necessarily distinct) points in $X$. Preferences need to be extended from $X$ to the set of subsets of $X$ of cardinality at most $m$. Ehlers assumes that each individual orders subsets by comparing his or her most-preferrred alternatives in these sets. For $m=2$, he has shown that the only social choice correspondence satisfying ACA, IIF, SP, and $\mathrm{IC}$ is the extreme peaks social choice correspondence. For each profile, this solution identifies the individuals with the smallest and largest peaks and then maximizes their preferences on each agenda. For $m>2$, Ehlers has shown that SP and IC are incompatible.

\section{Analytic preference domains}

We have seen in Examples 11 and 12 that Arrow's social welfare function axioms are inconsistent for the domain of monotone analytic preferences with no critical points when there are two or more public goods and for the domain of Euclidean spatial preferences. Le Breton and Weymark (2002) have shown that if either of these domains of analytic preferences is combined with an agenda domain consisting of compact sets with nonempty interiors, then IIF is vacuous on the combined agenda and preference domain, and this permits

\footnotetext{
${ }^{98} \mathrm{~A}$ preference that is strictly quasiconcave according to the definition in Section 3 need not be quasiconcave.
} 
the construction of social choice correspondences that satisfy the Arrovian axioms. We review their results in this section.

\subsection{Euclidean spatial preferences}

Recall that $\mathcal{E}$ denotes the set of all Euclidean spatial preferences on $\mathbb{R}_{+}^{m}$. In the spatial preference domain that we consider in this section, each individual is assumed to have a Euclidean spatial preference.

Euclidean Spatial Preference Domain. $\mathcal{D} \subseteq \mathcal{E}^{n}$.

The assumptions on the agenda domain are made precise in the following definition.

Full-Dimensional Compact Agenda Domain. For all $A \in \mathcal{A}, A$ is a compact set with a nonempty interior.

If we think of the preferences as belonging to legislators who must choose the quantities of various public goods, an agenda can be interpreted as the set of public goods allocations that are feasible given the resources at the legislators' disposal. As these resources are varied, we obtain different agendas.

With these domain assumptions, IIF is vacuous.

Lemma 6. If $X=\mathbb{R}_{+}^{m}$ with $m \geq 2$ and a social choice correspondence is defined on a full-dimensional compact agenda domain and a Euclidean spatial preference domain, then it satisfies IIF.

The basic idea of the proof is very simple. A Euclidean spatial preference is completely determined by the location of its ideal point. Further, the ideal point can be identified from a segment of an indifference contour by taking the point of intersection of the lines orthogonal to the indifference surface at two points in the segment. As a consequence, it is not possible to have two profiles coincide on an agenda unless they are identical.

With this result in hand, Le Breton and Weymark (2002) were able to establish the following possibility theorem.

Theorem 26. If $X=\mathbb{R}_{+}^{m}$ with $m \geq 2$, on any full-dimensional compact agenda domain and any Euclidean spatial preference domain, there exist social choice correspondences that satisfy ACA, IIF, SP, and ANON. 
The following example was used to establish Theorem 26.

Example 25. A Bergson-Samuelson social welfare function is a real-valued function defined on $n$-tuples of utilities. Let $W: \mathbb{R}^{n} \rightarrow \mathbb{R}$ be any continuous, symmetric, Bergson-Samuelson social welfare function, increasing in each of its arguments. For all $R \in \mathcal{E}$, a continuous utility function $U^{R}$ is chosen to represent $R$. Using the Bergson-Samuelson social welfare function $W$ and these representations of the individual preferences, the social welfare function $F: \mathcal{D} \rightarrow \mathcal{R}$ is defined by setting,

$$
x F(\mathbf{R}) y \leftrightarrow W\left[U^{R_{1}}(x), \ldots, U^{R_{n}}(x)\right] \geq W\left[U^{R_{1}}(y), \ldots, U^{R_{n}}(y)\right],
$$

for all $\mathbf{R} \in \mathcal{D}$ and all $x, y \in X$. The social choice correspondence $C: \mathcal{A} \times \mathcal{D} \rightarrow$ $X$ is defined by letting $C(A, \mathbf{R})$ be the set of best alternatives in $A$ according to the social preference $F(\mathbf{R})$. Formally, $C(A, \mathbf{R})=B(A, F(\mathbf{R}))$ for all $(A, \mathbf{R}) \in \mathcal{A} \times \mathcal{D}$. Because $W$ and $U^{R_{i}}, i \in N$, are continuous functions, $F(\mathbf{R})$ is a continuous ordering. Thus, $C$ is well-defined because each agenda is compact.

By Lemma 6, $C$ satisfies IIF. $C$ satisfies SP because $W$ is an increasing function. Because $W$ is symmetric in its arguments and the same utility function $U^{R}$ is used no matter who has the preference $R, C$ satisfies ANON. Because $C$ is rationalized by the social welfare function $F$, it satisfies ACA.

An attractive feature of Example 25 is that it provides a link between Arrovian social choice theory and traditional Bergson-Samuelson welfare economics. ${ }^{99}$ Because none of the binary agendas are feasible, the social welfare function used in this example does not satisfy IIA, thereby circumventing the social welfare function impossibility theorem for Euclidean spatial preferences discussed in Example 12.

\subsection{Monotone analytic preferences}

Le Breton and Weymark (2002) have also used the construction in Example 25 to establish a possibility theorem for monotone analytic preferences. Let $\mathcal{M}$ denote the set of all monotone analytic preferences preferences with no critical points on $\mathbb{R}_{+}^{m}$. Le Breton and Weymark assumed that the preference domain is any subset of $\mathcal{M}^{n}$. Thus, further restrictions, such as convexity of preferences, can be imposed on the preference domain.

\footnotetext{
${ }^{99}$ See also the related discussion in Pazner (1979).
} 
Monotone Analytic Preference Domain. $\mathcal{D} \subseteq \mathcal{M}^{n}$.

As above, each agenda is assumed to be a nonempty compact set with a nonempty interior. An agenda can be interpreted as being the set of feasible allocations of public goods obtainable from the economy's initial resources given the production possibility sets of the firms. Different agendas are obtained by varying the production technologies and/or the resource endowments. The compactness of an agenda follows from standard assumptions on firms' technologies that imply that the aggregate production possibilities set is closed and that only finite amounts of goods may be produced with the economy's resource endowment. Agendas can also be supposed to be convex and comprehensive, as would be the case if firms' technologies are convex and exhibit free disposal. It is important that the agendas are compact. On a noncompact, comprehensive agenda, the Pareto set is typically empty, making it impossible for a social choice correspondence to satisfy WP.

Consider two real-valued monotone analytic functions with no critical points defined on $\mathbb{R}_{++}^{m}$, with $m \geq 2$. Le Breton and Weymark have shown that if these functions are ordinally equivalent on an open subset of $\mathbb{R}_{++}^{m}$, then they are ordinally equivalent on all of $\mathbb{R}_{++}^{m}{ }^{100}$ Because the admissible agendas have nonempty interiors, it then follows that it is impossible for two distinct profiles in $\mathcal{M}^{n}$ to coincide on an agenda. Hence, IIF is vacuous.

Lemma 7. If $X=\mathbb{R}_{+}^{m}$ with $m \geq 2$ and a social choice correspondence is defined on a full-dimensional compact agenda domain and a monotone analytic preference domain, then it satisfies IIF.

Using Lemma 7, the social choice correspondence in Example 25 is easily shown to satisfy all the axioms of Theorem 26 on a full-dimensional compact agenda domain and a monotone analytic preference domain.

Theorem 27. If $X=\mathbb{R}_{+}^{m}$ with $m \geq 2$, on any full-dimensional compact agenda domain and any monotone analytic preference domain, there exist social choice correspondences that satisfy ACA, IIF, SP, and ANON.

As noted by Le Breton and Weymark, it is straightforward to construct a private goods version of Theorem 27. With private goods, this theorem is modified by (i) assuming that individuals are selfish and have preferences for own consumption that are monotone and analytic with no critical points and (ii) replacing ANON with PANON.

\footnotetext{
${ }^{100}$ This result is an ordinal version of the Analytic Continuation Principle for monotone analytic functions with no critical points.
} 


\section{Classical domains of spatial and economic prefer- ences}

The results discussed in the preceding section demonstrate that by combining an Arrow-inconsistent preference domain with natural restrictions on the admissible agendas, it is sometimes possible to obtain a social choice correspondence possibility theorem. However, there are spatial preferences that are not Euclidean and there are classical economic preferences that are not analytic. In this section, we review a number of results for larger domains of spatial and economic preferences. In Sections 16.1 and 16.2, we consider classical domains of economic preferences for exchange and production economies. In Section 16.3, we consider spatial preferences that are not limited to being Euclidean.

\subsection{Exchange economies}

Bailey (1979) questioned the relevance of Arrow's Theorem for economic problems on the grounds that economic agendas typically contain an infinite number of alternatives, whereas Arrow's proof of his theorem rests on an implicit assumption that all binary agendas are feasible. Bailey purported to provide an example of a social choice rule that satisfies all of the Arrow axioms for a class of exchange economies. Bailey's discussion of his example is quite informal and confuses the social welfare function and social choice correspondence formulations of Arrow's problem in a rather fundamental way. ${ }^{101}$ Below, we argue that Bailey's example is problematic and, as a consequence, misidentifies why Arrow's axioms are consistent for a domain of exchange economies. Nevertheless, Bailey was correct to question the relevance of Arrow's Theorem for exchange economies, as Donaldson and Weymark (1988) have demonstrated.

Consider an economy with $m \geq 2$ private goods. The set of alternatives is $X=\prod_{i=1}^{n} X_{i}$, where $X_{i}=\mathbb{R}_{+}^{m}$ for all $i \in N$. We consider two domains of economic preferences. The first is the classical private goods preference domain $\mathcal{C}_{p r}$ considered in Example 17. Recall that $\mathcal{C}_{p r}$ is the set of all profiles of selfish, continuous, strictly monotonic in own consumption, convex preference

\footnotetext{
${ }^{101}$ In fairness to Bailey, we note that some of this confusion is due to the fact that the independence condition stated as an axiom in Arrow (1951) is IIF, even though it is IIA that is used to establish Arrow's Theorem.
} 
orderings on $\mathbb{R}_{+}^{m n}$. Our second preference domain strengthens the assumptions on preferences by ruling out the possibility that an individual's induced private preference can have an indifference contour containing a consumption bundle with strictly positive components that intersects an axis.

Strongly Classical Private Goods Preference Domain. For all $i \in N, \mathcal{D}_{i}$ is the set of all selfish, continuous, strictly monotonic in own consumption, convex preference orderings on $\mathbb{R}_{+}^{m n}$ for which $x P_{i} y$ for all $R_{i} \in \mathcal{D}_{i}$, all $x \in \mathbb{R}_{+}^{m n}$ with $x_{j}^{i}>0$ for all $j \in\{1, \ldots, m\}$, and all $y \in \mathbb{R}_{+}^{m n}$ with $y_{j}^{i}=0$ for some $j \in\{1, \ldots, m\}$.

For each aggregate endowment $\omega \in \mathbb{R}_{++}^{m}$, the generalized Edgeworth box is $A(\omega)=\left\{x \in X \mid \sum_{i=1}^{n} x_{j}^{i} \leq \omega_{j}\right.$ for all $\left.j=1, \ldots, m\right\}$. Note that it is not assumed a priori that all of the endowment is consumed. The agenda domain is the set of all generalized Edgeworth boxes.

Edgeworth Box Agenda Domain. $\mathcal{A}=\left\{A(\omega) \mid \omega \in \mathbb{R}_{++}^{m}\right\} .{ }^{102}$

Donaldson and Weymark (1988) claimed that the Walrasian competitive equilibrium correspondence when the endowment is shared equally satisfies all of the Arrow axioms with ND strengthened to PANON on an Edgeworth Box agenda domain and a classical private goods preference domain. In order for their argument to be valid, we must use the slightly more restrictive preference domain described above.

Theorem 28. For a private goods economy with $X=\mathbb{R}_{+}^{m n}$ and $m \geq 2$, there exist social choice correspondences with an Edgeworth box agenda domain and a strongly classical private goods preference domain that satisfy ACA, IIF, $\mathrm{SP}$, and PANON. ${ }^{103}$

Example 26 defines the Walrasian correspondence from equal split and shows why it satisfies the assumptions of Theorem 28 .

Example 26. Suppose that the set of alternatives $X$, the agenda domain $\mathcal{A}$, and the preference domain $\mathcal{D}$ are defined as in Theorem 28. For each

\footnotetext{
${ }^{102}$ Note that in contrast with the exchange economies considered in Section 10.2, free disposal is permitted and consumption of goods is not required to be strictly positive.

${ }^{103}$ The weak and strong Pareto sets coincide in economies with classical economic preferences.
} 
aggregate endowment and admissible preference profile, the Walrasian correspondence from equal split is the social choice correspondence $C$ defined by setting the choice set equal to the set of all competitive equilibrium allocations obtainable from an initial equal division of the endowments. That is, $\omega / n$ is each person's endowment when the agenda is $A(\omega)$.

Because competitive equilibria exist in an exchange economy with classical economic preferences when each person has a positive endowment of every good, $C$ is well-defined. By the first Fundamental Theorem of Welfare Economics, all competitive equilibria are Pareto optimal. Because each person has the same endowment, permuting private preferences among individuals results in an economy that only differs from the original economy in the labelling of individuals, so PANON is also satisfied.

With preferences that are strictly monotonic in own consumption, Pareto optimality requires all of the resources be consumed. In order for $A\left(\omega^{1}\right)$ to be a strict subset of $A\left(\omega^{2}\right)$, it must be the case that $\omega_{j}^{1} \leq \omega_{j}^{2}$ for all $j$, with strict inequality for some $j$. Hence, for a given preference profile $\mathbf{R}$, the strong Pareto sets $\mathcal{P}^{s}\left(A\left(\omega^{1}\right), \mathbf{R}\right)$ and $\mathcal{P}^{s}\left(A\left(\omega^{2}\right), \mathbf{R}\right)$ do not intersect. Thus, $C$ trivially satisfies ACA.

It remains to show that IIF is satisfied. On the contrary, suppose that there exists an endowment $\omega \in \mathbb{R}_{++}^{m}$, profiles $\mathbf{R}^{1}, \mathbf{R}^{2} \in \mathcal{D}$ that coincide on $A(\omega)$, and an allocation $x$ for which $x \in C\left(A(\omega), \mathbf{R}^{1}\right)$ and $x \notin C\left(A(\omega), \mathbf{R}^{2}\right)$. Because $x$ is a Walrasian equilibrium from equal split for the profile $\mathbf{R}^{1}$, there exists a price vector $p \in \mathbb{R}_{++}^{n}$ such that for all $i \in N, p x^{i}=p \omega / n$ and $p y^{i}>p \omega / n$ if $y P_{i} x$. The assumptions on preferences imply that $x \in \mathbb{R}_{++}^{m n}$, so $x$ is in the interior of the generalized Edgeworth box $A(\omega)$. Because $x$ is not a Walrasian equilibrium from equal split for the profile $\mathbf{R}^{2}$, there exists a $k \in N$ and $y \in \mathbb{R}_{+}^{m n}$ such that $y P_{k}^{2} x$ and $p y^{k} \leq p \omega / n$. To satisfy our preference domain assumption, we must have $y^{k} \in \mathbb{R}_{++}^{m}$. Because preferences are convex and monotone in own consumption, $[\lambda y+(1-\lambda) x] P_{k}^{2} x$ for all $\lambda \in(0,1]$. Further, for such $\lambda, p\left[\lambda y^{k}+(1-\lambda) x^{k}\right] \leq p \omega / n$. Because $x$ is in the interior of $A(\omega)$, for positive $\lambda$ sufficiently close to zero, $[\lambda y+(1-\lambda) x]$ is also in the interior of $A(\omega)$. On $A(\omega), \mathbf{R}^{1}$ and $\mathbf{R}^{2}$ coincide. Hence, for such $\lambda$, we have $[\lambda y+(1-\lambda) x] P_{k}^{1} x$ and $p\left[\lambda y^{k}+(1-\lambda) x^{k}\right] \leq p \omega / n$, which contradicts the assumption that $x$ is a Walrasian equilibrium from equal split for the profile $\mathbf{R}^{1}$. Thus, $C$ satisfies IIF. ${ }^{104}$

\footnotetext{
${ }^{104}$ With the preference domain used by Donaldson and Weymark (1988), it is possible for an equilibrium allocation to lie on the boundary of a generalized Edgeworth box. For
} 
As in Section 15, the consistency of the axioms in Theorem 28 turns on one of the axioms being trivially satisfied. In this case, it is ACA that is vacuous, at least for Paretian social choice correspondences.

A related Arrovian possibility theorem was established by Grether and Plott (1982). They noted that when the preference domain is unrestricted and there are a finite number of alternatives, all of the Arrow axioms can be satisfied by setting each choice set equal to the weak Pareto set if the feasible agendas are pairwise disjoint. In their result, it is the pairwise disjointness of the feasible agendas that renders ACA vacuous. Because the resource constraints are not assumed to hold with equality, this is not the case in Example 26. ${ }^{105}$

There is a fixed aggregate endowment in the main example in Bailey (1979). A government allocates these resources to individuals, with the final allocation of goods determined by the competitive market mechanism. Bailey clearly regarded each distribution of resources as corresponding to a different agenda. However, how the aggregate endowment is distributed does not affect what is feasible for the economy, so it would seem that there is only one feasible set in his framework. Because the equilibrium allocations depend on the initial allocation of endowments to individuals, Bailey has not constructed an Arrovian social choice correspondence.

concreteness, suppose that there are just two goods and two individuals. If, for example, only person one receives a positive consumption of all goods at the equilibrium allocation $x$, person one's indifference curve containing $x$ consists of two linear segments with a kink at $x$, and the slope of this indifference curve outside the Edgeworth box has the same slope as the budget line, then changing person one's preferences over non-feasible alternatives can change the Walrasian equilibrium. This is not possible if the equilibrium allocation is in the interior of the Edgeworth box.

This problem does not arise with the constrained Walrasian correspondence from equal split. This correspondence is defined in the same way as the Walrasian correspondence from equal split except that, for every price vector, each person's budget set is the intersection of the Walrasian budget set with the set of consumption bundles that do not exceed the aggregate endowment $\omega$. This correspondence satisfies all of the axioms of Theorem 28 on an Edgeworth box agenda domain and a classical private goods preference domain. We are grateful to Marc Fleurbaey for this observation.

${ }^{105}$ The finiteness of the set of alternatives ensures that the Pareto sets in Grether and Plott's construction are nonempty. Nonemptyness of the Pareto sets in Example 26 follows from the compactness of the feasible agendas and the continuity of the individual preferences. 


\subsection{Production economies}

ACA is not, in general, vacuous in production economies. Nevertheless, Donaldson and Weymark (1988) have shown that the Arrow axioms are consistent in production economies. They considered both public goods and private goods economies.

In the case of private goods, the universal set of alternatives is the same as in the preceding subsection. In the public goods economy, there are $m \geq 2$ public goods and the set of alternatives is $X=\mathbb{R}_{+}^{m}$.

Donaldson and Weymark's possibility theorem is not very sensitive to the choice of preference domain, provided that all preferences are continuous. They assumed that the preference domain is the set of all profiles of continuous preferences in the public goods case and the set of all profiles of continuous selfish preferences in the private goods case. They noted that their theorem also holds with classical restrictions on preferences, and we state their result in this form. For private goods, we use the classical private goods preference domain $\mathcal{C}_{p r}$. For public goods, we use the classical public goods preference domain $\mathcal{C}_{p u}$ described in Example 8. Recall that $\mathcal{C}_{p u}$ is the set of all profiles of continuous, strictly monotonic, convex preference orderings on $X$.

In addition to the assumptions made about the agenda domain in Section 15 , it is assumed that all agendas are comprehensive.

Full-Dimensional Compact Comprehensive Agenda Domain. For all $A \in \mathcal{A}$, $A$ is a compact comprehensive set with a nonempty interior.

As in Section 15.2, an agenda is interpreted as being the set of feasible allocations of goods that can be made available to consumers from the economy's initial resources using the production possibility sets of the firms. With this interpretation of an agenda, comprehensiveness corresponds to assuming that goods can be freely disposed. Theorem 29 is valid with further restrictions on the agenda, such as convexity.

For both public and private goods economies, Donaldson and Weymark (1988) have shown that Arrow's axioms are consistent if the preference domain is classical and there is a full-dimensional compact comprehensive agenda domain. It is an open question whether ANON and PANON can be used instead of ND in this theorem.

Theorem 29. (a) For a public goods economy with $X=\mathbb{R}_{+}^{m}$ and $m \geq 2$, 
there exist social choice correspondences with a full-dimensional compact comprehensive agenda domain and a classical public goods preference domain that satisfy ACA, IIF, WP, and ND. (b) For a private goods economy with $X=\mathbb{R}_{+}^{m n}$ and $m \geq 2$, there exist social choice correspondences with a fulldimensional compact comprehensive agenda domain and a classical private goods preference domain that satisfy ACA, IIF, WP, and ND.

As in Example 23, there is a distinguished alternative in every admissible agenda. Here, this distinguished alternative is the origin. However, every admissible preference ranks the origin last and every agenda includes an infinite number of alternatives, so it is not possible to adapt Example 23 to show the consistency of Arrow's axioms in production economies. Although there do not exist more than one distinguished alternative in every admissible agenda, the example Donaldson and Weymark used to establish Theorem 29 exploits the fact that profiles can be partitioned in such a way that whenever two profiles coincide on an agenda, they must be in the same cell of the partition, as is the case in the construction (Example 24) Gibbard, Hylland, and Weymark (1987) used to show the consistency of the Arrow axioms when the agenda domain $\{\bar{x}, \bar{y}\}$-restricted. We present Donaldson and Weymark's public goods example. A private goods version of this example can be used to establish the second part of Theorem 29.

Example 27. Consider a public goods economy with $X=\mathbb{R}_{+}^{m}$ and $m \geq 2$ that satisfies the domain restrictions of Theorem 29. Let $\mathbf{R}^{*}$ be an arbitrary profile in $\mathcal{D}$. The preference domain is partitioned into the sets $\mathcal{D}_{1}$ and $\mathcal{D}_{2}$ as follows. For any profile $\mathbf{R} \in \mathcal{D}, \mathbf{R} \in \mathcal{D}_{1}$ if and only if $\mathbf{R}$ coincides with $\mathbf{R}^{*}$ in some neighbourhood of the origin. The social choice correspondence $C: \mathcal{A} \times \mathcal{D} \rightarrow X$ is defined by setting, for all $A \in \mathcal{A}, C(A, \mathbf{R})=B\left(A, R_{1}\right)$ for all $\mathbf{R} \in \mathcal{D}_{1}$ and $C(A, \mathbf{R})=B\left(A, R_{2}\right)$ for all $\mathbf{R} \in \mathcal{D}_{2}$. That is, person one is a strong dictator for the profiles in $\mathcal{D}_{1}$ and person two is a strong dictator for the profiles in $\mathcal{D}_{2}$.

Using essentially the same reasoning as in Example 24, $C$ satisfies ACA, WP, and ND. Because any admissible agenda $A$ includes a neighbourhood of the origin, if two profiles agree on $A$, they must (i) either both agree with $\mathbf{R}^{*}$ on some neighbourhood of the origin or (ii) neither of them agrees with $\mathbf{R}^{*}$ on any neighbourhood of the origin. In either case, the same dictator is used for both profiles, so IIF is satisfied. ${ }^{106}$ Note that that $C$ is rationalized by a

\footnotetext{
${ }^{106}$ If a serial dictatorship is used for each cell of the partition, SP is also satisfied. Ev-
} 
social welfare function that satisfies all of the Arrow axioms except IIA. ${ }^{107}$

Consider a public goods economy with $X=\mathbb{R}_{+}^{m}$ and $m \geq 2$. For all $(p, w) \in \mathbb{R}_{++}^{m+1}$, let $Y(p, w)=\{x \in X \mid p x \leq w\} . Y(p, w)$ can be interpreted as being the set of feasible allocations for a production economy with a linear production possibility set.

Complete Linear Comprehensive Agenda Domain. $A \in \mathcal{A}$ if and only if $A=Y(p, w)$ for some $(p, w) \in \mathbb{R}_{++}^{m+1}$.

With this agenda domain, there is little scope for ACA to play a role if preferences are continuous and monotonic and the social choice correspondence is Paretian. WP implies that all Pareto optimal alternatives are contained in the upper boundary of an agenda. If one "linear" agenda is a strict subset of another, either their upper boundaries do not intersect or, if they do, all the alternatives they have in common lie on the boundary of $\mathbb{R}_{+}^{m}$. Thus, if the preference domain is restricted in such a way that weak Pareto sets only include alternatives with positive consumption of all goods, ACA is vacuous. The following preference domain has this feature.

Strongly Convex Classical Public Goods Preference Domain. $\mathcal{D}$ is the set of all profiles of continuous preference orderings on $\mathbb{R}_{+}^{m}$ that are strictly monotonic on $\mathbb{R}_{++}^{m}$ and strictly convex relative to $\mathbb{R}^{m}$.

The requirement that each admissible preference is strictly convex relative to $\mathbb{R}^{m}$ rules out any indifference contour that contains an alternative with strictly positive components from intersecting an axis.

Le Breton (1997) has observed that with this kind of preference domain and a complete linear comprehensive agenda domain, it is possible to construct social choice correspondences that satisfy all of the Arrow axioms, with ND strengthened to ANON.

Theorem 30. If $X=\mathbb{R}_{+}^{m}$ with $m \geq 2$, there exist social choice correspondences with a complete linear comprehensive agenda domain and a strongly convex classical public goods preference domain that satisfy ACA, IIF, WP, and ANON.

eryone can be a dictator on some cell if more reference profiles are used to partition the preference domain into $n$ or more cells.

${ }^{107}$ Campbell (1995) has shown that the Arrow axioms are inconsistent if the preference domain in this example is combined with an agenda domain that includes all finite subsets of $X$ of cardinalities $k$ and $k+1$ for some $k \geq 2$, but no subset with a smaller cardinality. 
Le Breton did not provide an explicit example of such a social choice correspondence. We provide one in Example 28.

Example 28. Consider a public goods economy with $X=\mathbb{R}_{+}^{m}$ and $m \geq 2$ and a social choice correspondence $C$ that satisfies the domain assumptions of Theorem 30. Let $C$ be defined by setting $C(Y(p, w), \mathbf{R})=\mathcal{P}^{w}(Y(p, w), \mathbf{R})$ for all $(p, w) \in \mathbb{R}_{++}^{m+1}$ and all $\mathbf{R} \in \mathcal{D}$. In other words, the social choice correspondence always chooses the whole weak Pareto set, as in the GretherPlott example discussed in Section 16.1.

\subsection{General spatial preferences}

The distinguishing features of a spatial preference are that it is convex and it has a bliss point. Duggan (1996) has shown that the Arrow axioms are inconsistent when the preference domain includes all profiles of spatial preferences and the agenda domain is the set of compact convex subsets of the universal set of alternatives $X$ when $X$ is a multidimensional convex subset of a Euclidean space. We consider Duggan's theorem in this section.

More precisely, with $X$ as described above, a spatial preference $R$ on $X$ is a continuous, strictly convex ordering with a bliss point. Let $\mathcal{S}$ denote the set of all spatial preferences. ${ }^{108}$ Clearly, $\mathcal{E} \subset \mathcal{S}$. Duggan assumed that all profiles of spatial preferences are admissible.

Unrestricted Spatial Preference Domain. $\mathcal{D}=\mathcal{S}^{n}$.

Duggan's agenda domain is the set of all compact convex subsets of $X$.

Complete Compact Convex Agenda Domain. $\mathcal{A}$ is the set of compact convex subsets of $X$.

Note that any spatial preference in $\mathcal{S}$ has a unique maximum on any compact convex agenda.

Aside from the trivial agendas that contain only one alternative, each admissible agenda contains an infinite number of alternatives. Duggan noted that the proof of his theorem only requires the agenda domain to include the agendas formed by taking the convex hulls of any pair or triple of alternatives. However, it is essential that the domain includes the convex hull of any

\footnotetext{
${ }^{108}$ Because a spatial preference is required to be continuous, if $X$ is an interval of the real line, $\mathcal{S}$ is a strict subset of the set of single-peaked preferences $\mathcal{S}_{P}$.
} 
pair of alternatives, and such sets do not have an interior. In contrast, the possibility theorem for Euclidean spatial preferences presented in Section 15.1 and the possibility theorems for economic preference domains discussed above all assume that every agenda has a nonempty interior. This assumption plays an important role in the proofs of these results.

Theorem 31 is Duggan's impossibility theorem for spatial preferences.

Theorem 31. If $X$ is a convex subset of a Euclidean space and the dimension of $X$ is at least two, there is no social choice correspondence with a complete compact convex agenda domain and an unrestricted spatial preference domain that satisfies ACA, IIF, WP, and ND.

The proof we presented in Section 12.2 of the choice-theoretic version of Arrow's Theorem begins by rationalizing the social choice correspondence by a social welfare function and then showing that this social welfare function satisfies all of Arrow's original axioms, which is impossible. Because Duggan's agenda domain is not closed under finite unions, Hansson's Theorem does not apply, so ACA does not guarantee that the social choice correspondence can be rationalized. Thus, a different proof strategy is required to establish Theorem 31. Duggan's proof of this theorem is an ingenious adaptation of the proof strategy described in Section 2 for the social welfare function version of Arrow's Theorem. Duggan's proof is too long to reproduce here, so we limit ourselves to a discussion of its main features.

Let $C$ be a social choice correspondence satisfying Duggan's domain assumptions and all of the Arrow axioms except ND. For any subset $Y$ of $X$, let ch $(Y)$ denote the convex hull of $Y$. Although no pair of distinct alternatives is an admissible agenda, the convex hull of any pair is. It is these sets that Duggan used to make binary comparisons. Note that any admissible preference is single-peaked on $c h(\{x, y\})$. Duggan defined his decisiveness relations for $\{x, y\}$ in terms of the social choice from $\operatorname{ch}(\{x, y\})$ when the individual preference peaks are at the endpoints of this line segment. Specifically, for any ordered pair $(x, y) \in X^{2}$, (i) $G \subseteq N$ is almost decisive for the ordered pair $(x, y)$ if $C(\operatorname{ch}(\{x, y\}), \mathbf{R})=\{x\}$ when $B\left(\operatorname{ch}(\{x, y\}), R_{i}\right)=\{x\}$ for all $i \in G$ and $B\left(\operatorname{ch}(\{x, y\}), R_{i}\right)=\{y\}$ for all $i \notin G$ and (ii) $G$ is decisive for the ordered pair $(x, y)$ if $C(\operatorname{ch}(\{x, y\}), \mathbf{R})=\{x\}$ when $B\left(\operatorname{ch}(\{x, y\}), R_{i}\right)=\{x\}$ for all $i \in G$. By IIF, if a group is (almost) decisive for $(x, y)$, then it cannot be (almost) decisive for $(y, x)$.

A group $G \subseteq N$ is proper if $\emptyset \neq G \neq N$. For any $x, y, z \in X$ that are not colinear, Duggan has shown that if a proper group $G$ is almost decisive for 
$(x, y)$, then it is also almost decisive for $(y, z)$. This is done by investigating the relationship between the social choice from $\operatorname{ch}(\{x, y, z\})$ and the choices from the edges of $\operatorname{ch}(\{x, y, z\})$ when the Pareto set of $\operatorname{ch}(\{x, y, z\})$ is contained in $\operatorname{ch}(\{x, y\})$. A similar argument then shows that $G$ is also almost decisive for $(w, z)$ when $x, z$, and $w$ are not colinear. Because $X$ is at least two dimensional, these two results imply that if a proper group $G$ is almost decisive for some pair of distinct alternatives, then it is almost decisive for all pairs. Once this has been established, it is not too difficult to show that $G$ is decisive for all pairs of alternatives.

The preceding argument shows that $G$ has the power to determine the social choice on line segments when the members of $G$ all agree that one end of the line segment is the best feasible alternative. The next step in the argument shows that $G$ also has the power to determine the social choice on any feasible agenda $A$ if the members of $G$ agree about what is best in $A$. Duggan called a group $G$ for which $C(A, \mathbf{R})=\{x\}$ whenever $B\left(A, R_{i}\right)=\{x\}$ for all $i \in G$ an oligarchy. A dictator is a one-person oligarchy. By WP, $N$ is an oligarchy. The rest of the proof is devoted to showing that the smallest oligarchy contains only one person. Hence, $C$ is dictatorial.

As previously noted, Duggan's proof strategy requires that the line segment joining any two distinct alternatives in $X$ is an admissible agenda. It is an open question whether Arrow's axioms are consistent for the preference domain $\mathcal{S}^{n}$ when the agenda domain is the set of all compact convex subsets of $X$ that have nonempty interiors.

\section{Independence of Pareto Irrelevant Alternatives}

The example (Example 27) Donaldson and Weymark (1988) used to establish the consistency of Arrow's axioms in their possibility theorem for production economies (Theorem 29) has the property that whose preference is used to determine the choice set depends on properties of the preference profile in an arbitrarily small neighbourhood of the origin. With monotone preferences and a full-dimensioned agenda, none of these alternatives are Pareto optimal. Donaldson and Weymark have suggested that preferences for Pareto dominated alternatives should be irrelevant when determining what to choose from an agenda, a property they call Independence of Pareto Irrelevant Al-

ternatives. In this section, we review the theorems Donaldson and Weymark (1988) and Duggan (1996) have established using this independence axiom. 
With Independence of Pareto Irrelevant Alternatives, each choice set only depends on the restriction of the preference profile to the Pareto set. There are two versions of this axiom, one based on weak Pareto optimality and one based on strong Pareto optimality.

Independence of Weakly Pareto Irrelevant Alternatives (WPIIA). For all $A \in$ $\mathcal{A}$ and all $\mathbf{R}^{1}, \mathbf{R}^{2} \in \mathcal{D}$, if $\mathcal{P}^{w}\left(A, \mathbf{R}^{1}\right)=\mathcal{P}^{w}\left(A, \mathbf{R}^{2}\right)$ and $\mathbf{R}^{1}$ and $\mathbf{R}^{2}$ coincide on this common Pareto set, then $C\left(A, \mathbf{R}^{1}\right)=C\left(A, \mathbf{R}^{2}\right)$.

Independence of Strongly Pareto Irrelevant Alternatives (SPIIA). For all $A \in$ $\mathcal{A}$ and all $\mathbf{R}^{1}, \mathbf{R}^{2} \in \mathcal{D}$, if $\mathcal{P}^{s}\left(A, \mathbf{R}^{1}\right)=\mathcal{P}^{s}\left(A, \mathbf{R}^{2}\right)$ and $\mathbf{R}^{1}$ and $\mathbf{R}^{2}$ coincide on this common Pareto set, then $C\left(A, \mathbf{R}^{1}\right)=C\left(A, \mathbf{R}^{2}\right)$.

Note that, in general, the second axiom is more demanding than the first. Both of these axioms imply IIF on any domain for which the Pareto sets are always nonempty.

When the agenda domain consists of the nonempty finite subsets of $X$, because, for any admissible preference profile and any admissible agenda, each Pareto set is also feasible, ACA, IIF, and WP (resp. SP) imply WPIIA (resp. SPIIA). ${ }^{109}$ As a consequence, regardless of the preference domain, with an unrestricted finite feasible set agenda domain, the implications of IIF and WPIIA are the same when combined with Arrow's other axioms. This is not the case in production economies, as Example 27 demonstrates.

\subsection{Nonconvex agendas}

For the public goods economy considered in Theorem 29, Donaldson and Weymark (1988) have shown that replacing IIF with WPIIA results in an impossibility theorem if the agenda domain is enlarged to the set of all compact and comprehensive subsets of $X$.

Complete Compact Comprehensive Agenda Domain. $A \in \mathcal{A}$ if and only if $A$ is a compact comprehensive subset of $X$.

Note that this agenda domain includes sets with an empty interior.

Recall that for any $x, y \in \mathbb{R}_{+}^{m}$, their corner set $x_{\downarrow} \cup y_{\downarrow}$ is obtained by taking the union of the comprehensive sets of $x$ and $y$. Because agendas

${ }^{109}$ The finiteness assumption ensures that the Pareto sets are nonempty. 
have not been assumed to be convex, the corner set generated by any pair of alternatives is an admissible agenda. It is these agendas that permit Donaldson and Weymark to make binary comparisons.

Theorem 32 is Donaldson and Weymark's public goods impossibility theorem.

Theorem 32. If $X=\mathbb{R}_{+}^{m}$ with $m \geq 2$, there is no social choice correspondence with a complete compact comprehensive agenda domain and a classical public goods preference domain that satisfies ACA, WPIIA, WP, and ND.

Proof. On the contrary, suppose that there exists a social correspondence $C: \mathcal{A} \times \mathcal{D} \rightarrow X$ that satisfies all the assumptions of the theorem. The agenda domain is closed under finite unions, so by Hansson's Theorem (Theorem 18), $C$ can be rationalized by a social welfare function $F: \mathcal{D} \rightarrow \mathcal{R}$.

Consider any $x, y \in X$ and any $\mathbf{R} \in \mathcal{D}$. By the continuity and monotonicity of preferences and the compactness of $x_{\downarrow} \cup y_{\downarrow}$, the Pareto set $\mathcal{P}^{w}\left(x_{\downarrow} \cup y_{\downarrow}, \mathbf{R}\right)$ is a nonempty subset of $\{x, y\}$. Hence, WP implies that $C\left(x_{\downarrow} \cup y_{\downarrow}, \mathbf{R}\right) \subseteq$ $\{x, y\}$. It follows from this observation that $F$ is uniquely determined from the choices out of corner sets as follows: for all $x, y \in X$ and all $\mathbf{R} \in \mathcal{D}$, $x F(\mathbf{R}) y \leftrightarrow x \in C\left(x_{\downarrow} \cup y_{\downarrow}, \mathbf{R}\right)$.

If $\mathbf{R}^{1}$ and $\mathbf{R}^{2}$ coincide on $\{x, y\}$, then $\mathcal{P}^{w}\left(x_{\downarrow} \cup y_{\downarrow}, \mathbf{R}^{1}\right)=\mathcal{P}^{w}\left(x_{\downarrow} \cup y_{\downarrow}, \mathbf{R}^{2}\right)$. Hence, by WPIIA, $C\left(x_{\downarrow} \cup y_{\downarrow}, \mathbf{R}^{1}\right)=C\left(x_{\downarrow} \cup y_{\downarrow}, \mathbf{R}^{2}\right)$. Because $F$ rationalizes $C, F\left(\mathbf{R}^{1}\right)$ and $F\left(\mathbf{R}^{2}\right)$ therefore coincide on $\{x, y\}$; i.e., $F$ satisfies IIA.

If $x P_{i} y$ for all $i \in N$, then $x$ is the unique Pareto optimal alternative in $x_{\downarrow} \cup y_{\downarrow}$. Thus, $C\left(x_{\downarrow} \cup y_{\downarrow}, \mathbf{R}\right)=\{x\}$, from which it follows that $F$ satisfies WP.

Suppose that $d \in N$ is a dictator for $F$. Because $C$ is nondictatorial, there exists an $A \in \mathcal{A}, x, y \in A$, and an $\mathbf{R} \in \mathcal{D}$ such that $x P_{d} y$, but $y \in C(A, \mathbf{R})$. By ACA, $y \in C\left(x_{\downarrow} \cup y_{\downarrow}, \mathbf{R}\right) .{ }^{110}$ Because $F$ rationalizes $C$, $d$ is a dictator for $F$, and $x P_{d} y$, we must have $C\left(x_{\downarrow} \cup y_{\downarrow}, \mathbf{R}\right)=\{x\}$, a contradiction. Hence, $F$ satisfies ND.

We have shown that $F$ satisfies all of the assumptions of the Kalai-MullerSatterthwaite Theorem (Theorem 5), which is impossible.

This proof strategy is similar to the one used to prove the choice-theoretic version of Arrow's Theorem. The proof begins by assuming that all of the

\footnotetext{
${ }^{110}$ Even if $A$ has a nonempty interior, $x_{\downarrow} \cup y_{\downarrow}$ need not, which is why the agenda domain is not restricted to sets with nonempty interiors.
} 
axioms can be satisfied. Using Hansson's Theorem, ACA implies that the social choice correspondence is rationalized by a social welfare function. The choices from corner sets are used to uniquely identify this function, whereas the choice from pairs of alternatives were used to do this in the proof of Arrow's Theorem. This social welfare function is then shown to satisfy all of the Arrow social welfare function axioms. Finally, a social welfare function impossibility theorem is used to show that this is impossible. Here, it is the Kalai-Muller-Satterthwaite Theorem, not Arrow's original impossibility theorem that establishes the contradiction.

Donaldson and Weymark (1988) have established the following private goods analogue of Theorem 32 .

Theorem 33. If $X=\mathbb{R}_{+}^{m n}$ with $m \geq 2$, there is no social choice correspondence with a complete compact comprehensive agenda domain and a classical private goods preference domain that satisfies ACA, SPIIA, SP, and ND.

Because classical private goods preferences are only strictly monotonic in own consumption, for a profile $\mathbf{R}$, one cannot, in general, infer that the weak Pareto set for a corner set $x_{\downarrow} \cup y_{\downarrow}$ is contained in $\{x, y\}$. However, the strong Pareto set is. Thus, by strengthening WP to SP and using SPIIA instead of WPIIA, the argument used to prove Theorem 32 shows that a social choice correspondence satisfying the assumptions of Theorem 33 can be rationalized by a social welfare function satisfying the Arrow axioms, which is impossible by Theorem 16 on effective social welfare functions.

\subsection{Convex agendas}

Nonconvex agendas play two roles in the proof of Theorem 32. First, if only convex agendas are feasible, it would not be possible to use Hansson's Theorem to show that the social choice correspondence is rationalizable. Second, corner sets are needed to isolate pairs of alternatives, and so make binary comparisons. As a consequence, Donaldson and Weymark's proof strategy is ill-suited to deal with convex agendas.

Donaldson and Weymark (1988) and Duggan (1996) have investigated the consistency of the axioms in Theorem 32 in convex production economies. Donaldson and Weymark have shown that these axioms (with ND strengthened to ANON) are consistent when there are only two public goods, whereas Duggan has shown that they are not consistent when there are more than two goods. Their agenda domains are restricted to include only convex sets, 
as would be the case if all firms' technologies are convex. Preferences are required to be strictly convex, not merely convex. ${ }^{111}$

Complete Compact Comprehensive Convex Agenda Domain. $A \in \mathcal{A}$ if and only if $A$ is a compact, comprehensive, convex subset of $X$.

Strictly Convex Classical Public Goods Preference Domain. $\mathcal{D}$ is the set of all profiles of continuous, strictly monotonic, strictly convex preference orderings on $X$.

In Donaldson and Weymark's possibility theorem, ND is strengthened to ANON.

Theorem 34. If $X=\mathbb{R}_{+}^{2}$, there exist social choice correspondences with a complete compact comprehensive convex agenda domain and a strictly convex classical public goods preference domain that satisfies ACA, WPIIA, WP, and ANON.

With these domain restrictions, on the upper boundary of any agenda, everyone has a single-peaked preference. This observation was used by Donaldson and Weymark to construct the following example satisfying all of the assumptions of Theorem 34 .

Example 29. Consider a two-good public goods economy with $X=\mathbb{R}_{+}^{2}$ that satisfies the domain restrictions of Theorem 34. The social choice correspondence $C: \mathcal{A} \times \mathcal{D} \rightarrow X$ is defined by setting, for all $A \in \mathcal{A}$ and all $\mathbf{R} \in \mathcal{D}, C(A, \mathbf{R})=\left\{x \in A \mid x_{1}=\max _{i \in N} B_{1}\left(A, R_{i}\right)\right\}$, where $B_{1}\left(A, R_{i}\right)$ is the first component of the unique alternative that maximizes $R_{i}$ on $A$. On the upper boundary of $A$, everyone has single-peaked preferences. The rightmost of the individual peaks is the social choice. In other words, one of the class of generalized median social choice functions introduced by Moulin (1980) and discussed in Section 3 is used to determine each choice set.

The construction of $C$ for the case of three individuals is illustrated in Figure 13. Person one's best choices in the agendas $A^{1}$ and $A^{2}$ are $a$ and $b$, respectively. Person two's best choices in the agendas $A^{1}$ and $A^{2}$ are $c$ and $d$, respectively. Person three's best choice in both agendas is $e$. For this profile of preferences, the social choice out of both $A^{1}$ and $A^{2}$ is $e$.

\footnotetext{
${ }^{111}$ Alternatively, in Donaldson and Weymark's theorem, preferences need only be assumed to be convex if the agendas are assumed to be strictly convex.
} 
It is easy to verify that $C$ satisfies WPIIA, WP, and ANON. For a given profile, suppose that the agenda shrinks from, say, $A^{2}$ to $A^{1}$, but the original choice remains feasible. Anyone whose peak is still feasible has the same peak on $A^{1}$ as on $A^{2}$. Because the rightmost of the original peaks remains feasible, the convexity of preferences ensures that nobody's peak moves to the right of the original choice, as illustrated in Figure 13. Hence, ACA is satisfied.

Le Breton (1997) has observed that the social choice correspondence in this example can be rationalized by a social welfare function. Using the Hausdorff metric [see Munkres (1975)] to measure the distance between sets, for a given profile $\mathbf{R} \in \mathcal{D}$, for any sequence of agendas $A^{1}, A^{2}, \ldots$ in $\mathcal{A}$ for which $A^{k} \rightarrow A \in \mathcal{A}$ and $\mathcal{P}^{w}\left(A^{k}, \mathbf{R}\right) \rightarrow \mathcal{P}^{w}(A, \mathbf{R})$, it can be shown that $C\left(A^{k}, \mathbf{R}\right) \rightarrow C(A, \mathbf{R})$. It follows from a theorem of Peters and Wakker (1991) that when this Pareto continuity condition is satisfied on the agenda domain of Example 29, $C$ is rationalized by a social welfare function. Peters and Wakker's rationalizability theorem does not generalize to more goods. ${ }^{112}$

Duggan (1996) has shown that Arrow's axioms are inconsistent on a complete compact comprehensive convex agenda domain and a strictly convex classical public goods preference domain when there are three or more goods. Thus, the restriction to two goods in Theorem 34 is essential.

Theorem 35. If $X=\mathbb{R}_{+}^{m}$ with $m \geq 3$, there does not exist a social choice correspondence with a complete compact comprehensive convex agenda domain and a strictly convex classical public goods preference domain that satisfies ACA, WPIIA, WP, and ND.

Before proving Theorem 35, it is useful to provide an overview of Duggan's proof strategy. First, Duggan's spatial impossibility theorem (Theorem 31) is used to show that there is a dictator for the subdomain of agendas whose upper boundaries all lie in the same hyperplane,. A connection argument is then used to show that the same person is dictator on agendas whose upper boundaries are contained in different hyperplanes. Finally, ACA is used to show that this individual is a dictator on agendas whose upper boundaries are not contained in a hyperplane.

\footnotetext{
${ }^{112}$ Peters and Wakker's theorem is for single-valued choice correspondences. With singlevaluedness, as they show, if the agenda domain is closed under intersections, ACA is a necessary and sufficient condition for a choice correspondence to be rationalized by a binary relation (which need not be an ordering) on $A$.
} 
Proof of Theorem 35. Let $C: \mathcal{A} \times \mathcal{D} \rightarrow X$ be a social choice correspondence satisfying all of the assumptions of the theorem. For any $x \in \mathbb{R}_{+}^{m}$ and any $p \in \mathbb{R}_{++}^{m}$, let $H_{x}(p)$ denote the restriction to $\mathbb{R}_{+}^{m}$ of the hyperplane containing $x$ that is normal to $p$. For any compact convex subset $A$ of $H_{x}(p)$, let $A_{\downarrow}=$ $\cup_{x \in A} x_{\downarrow} \cdot A_{\downarrow}$ is an admissible agenda and its upper boundary is $A$. Note that any admissible preference $R$ is spatial when restricted to $H_{x}(p)$ and that $R$ has a unique maximum on $A_{\downarrow}$. Person $d \in N$ is a dictator for $H_{x}(p)$ if $C\left(A_{\downarrow}, \mathbf{R}\right)=B\left(A_{\downarrow}, R_{d}\right)$ for all compact convex $A \subseteq H_{x}(p)$ and all $\mathbf{R} \in \mathcal{D}$.

Consider any $x \in \mathbb{R}_{+}^{m} \backslash\left\{0_{m}\right\}$ and any $p \in \mathbb{R}_{++}^{m}$. Let $\mathcal{A}^{*}$ and $\mathcal{D}^{*}$ denote the restrictions of the agenda and preference domains to $H_{x}(p) . \mathcal{A}^{*}$ is a complete compact convex agenda domain and $\mathcal{D}^{*}$ is an unrestricted spatial preference domain. A social choice correspondence $C^{*}: \mathcal{A}^{*} \times \mathcal{D}^{*} \rightarrow H_{x}(p)$ can be defined by setting $C^{*}\left(A, \mathbf{R}^{*}\right)=C\left(A_{\downarrow}, \mathbf{R}\right)$ for all $A \in \mathcal{A}^{*}$ and all $\mathbf{R} \in \mathcal{D}$, where $\mathbf{R}^{*}$ is the restriction of $\mathbf{R}$ to $H_{x}(p)$. Because the Pareto set $\mathcal{P}^{w}\left(A_{\downarrow}, \mathbf{R}\right)$ is contained in $A$ when $A \subseteq H_{x}(p)$, WP and WPIIA ensure that $C^{*}$ is well-defined. It is easy to verify that $C^{*}$ satisfies ACA, IIF, and WP. Hence, by Theorem 31, $C^{*}$ is dictatorial, which implies that there is a dictator $d$ for $C$ on $H_{x}(p)$.

Consider any $x, y \in \mathbb{R}_{+}^{m} \backslash\left\{0_{m}\right\}$ and any $p, q \in \mathbb{R}_{++}^{m}$. Simple geometry confirms that it is possible to find a $z \in \mathbb{R}_{+}^{m} \backslash\left\{0_{m}\right\}$ and an $r \in \mathbb{R}_{++}^{m}$ such that $H_{z}(r)$ intersects both $H_{x}(p)$ and $H_{y}(q)$. Further, $H_{z}(r) \cap H_{x}(p)$ and $H_{z}(r) \cap H_{y}(q)$ are at least one-dimensional. There must be a dictator on each of these three sets and the overlap between $H_{x}(p)$ and $H_{z}(r)$ and between $H_{z}(r)$ and $H_{y}(q)$ implies that the same person is the dictator in all three cases. Hence, $d$ is a dictator on $H_{w}(s)$ for all $w \in \mathbb{R}_{+}^{m} \backslash\left\{0_{m}\right\}$ and all $s \in \mathbb{R}_{++}^{m} \cdot{ }^{113}$

Now consider any $A \in \mathcal{A}$ with $A \neq\left\{0_{m}\right\}$ and any $\mathbf{R} \in \mathcal{D}$. Let $x$ be the unique maximum of $R_{d}$ on $A$. Because $A$ is compact, convex, and comprehensive and $R_{d}$ is continuous, strictly monotonic, and convex, there exists a $p \in \mathbb{R}_{++}^{m}$ such that $H_{x}(p)$ is a separating hyperplane for $A$ and the upper contour set of $R_{d}$ at $x$. Because $d$ has strictly convex preferences and is a dictator for $H_{x}(p), C\left(H_{x}(p)_{\downarrow}, \mathbf{R}\right)=\{x\}$. ACA then implies that $C(A, \mathbf{R})=\{x\}$. Because the choice is trivial when $A=\left\{0_{m}\right\}, d$ is therefore a dictator for $C$.

WPIIA and SPIIA have not been investigated in private goods economies when only convex agendas are admissible.

\footnotetext{
${ }^{113}$ Note that it has now been established that the axioms are inconsistent on a complete linear agenda domain and a strictly convex classical public goods preference domain.
} 
Except when there are only two public goods and all agendas are convex, the theorems in this section are all negative. Duggan (1996) and Le Breton (1997) have argued that these results illustrate the undue restrictiveness of WPIIA and SPIIA and they should not be used to conclude that it is impossible to construct satisfactory social choice correspondences for production economies. Le Breton used the following example to make his point.

Example 30. Consider a public goods economy with $X=\mathbb{R}_{+}^{m}$ and $m \geq 2$. As in Example 28, suppose that there is a complete linear agenda domain. If everyone is given an equal share of the economy's wealth $w$ and given access to the linear technology defined by $p$, an individual with preference $R$ would choose $B(Y(p, w / n), R)$. Le Breton has suggested that fairness requires the social choice correspondence to guarantee that no individual is ever worse off than if he or she had an equal right to $w$. That is, with the agenda $Y(p, w)$ and profile $\mathbf{R}$, the choice set should be contained in $\cap_{i \in N} U\left(p, w, R_{i}\right)$, where $U\left(p, w, R_{i}\right)=\left\{x \in Y(p, w) \mid x R B\left(Y(p, w / n), R_{i}\right)\right\}$.

Suppose that $\mathcal{D}$ is a strictly convex classical public goods preferences domain. Define the social choice correspondence $C$ by setting $C(Y(p, w), \mathbf{R})=$ $B\left(\cap_{i \neq 1} U\left(p, w, R_{i}\right), R_{1}\right)$ for all $(p, w) \in \mathbb{R}_{++}^{m+1}$ and all $\mathbf{R} \in \mathcal{D}$. That is, person one's preferences are maximized subject to the constraint that none of the other individuals is worse off than they would be with an equal claim on the resources. $C$ satisfies all of the Arrow axioms and the fairness condition described above, but it does not satisfy WPIIA.

\section{Concluding remarks}

In this survey, we have considered the consistency of Arrow's axioms when his unrestricted domain assumptions are replaced by domain conditions that incorporate the restrictions on agendas and preferences encountered in economic environments. For social welfare functions, we have seen that economic domain restrictions do not provide a satisfactory way of avoiding Arrovian social choice impossibilities, except when the set of alternatives is one-dimensional and preferences are single-peaked. In contrast, for social choice correspondences, a number of economic domains have been identified for which the Arrow axioms are consistent.

With a social welfare function, a domain restriction only specifies what profiles of individual preferences are admissible. A unifying theme of Part I is that if a preference domain is Arrow-inconsistent, then this can gener- 
ally be established using some version of the local approach introduced by Kalai, Muller, and Satterthwaite (1979). This methodology has been used by Kalai, Muller, and Satterthwaite (1979), Bordes and Le Breton (1989; 1990b), and Redekop (1991; 1993a; 1993c; 1996) to identify sufficient conditions for a preference domain to be Arrow-inconsistent. The exact form of these conditions depends on whether the model being considered has public or private goods (or both). We have illustrated the power of this approach by showing how it can be used to prove that a number of domains of interest are saturating, which is Kalai, Muller, and Satterthwaite's sufficient condition for Arrow-inconsistency for public alternatives. The difficulty of determining whether a particular domain is saturating or not depends on the domain being considered, but the general principles being applied do not.

The local approach involves identifying free pairs and free triples and showing how the free pairs can be connected to each other using a chain of overlapping free triples. Even when not every pair of free pairs can be connected, we have shown that the application of the local approach can sometimes be used to infer a great deal about the structure of an Arrovian social welfare function, as is the case in our ordinal probability example (Example 15). Our discussion of exchange economies in Section 10.2 shows that it is even possible to adapt the local approach to show the Arrowinconsistency of a specific domain in which free triples fail to exist. Thus, the local approach can be viewed as being the fundamental tool for determining whether a preference domain is Arrow-inconsistent. ${ }^{114}$

The critical assumption that permits the application of the local approach is Independence of Irrelevant Alternatives. Mayston (1980) has noted that this axiom precludes considering individual marginal rates of substitution (when they are well-defined) in deciding how to socially rank a pair of alternatives, as it is not possible to calculate marginal rates of substitution without considering other "irrelevant" alternatives. In economic models, it therefore seems appropriate to consider weaker formulations of Arrow's independence condition, formulations that permit marginal rates of substitution at an alternative $x$ or the indifference surface containing $x$ to be relevant fea-

\footnotetext{
${ }^{114}$ An alternative to the local approach has been proposed by Saari (1991) to investigate the consistency of axioms for aggregation problems, including preference aggregation problems of the kind considered in Part I. Saari's approach utilizes a discrete version of a calculus argument to identify restrictions on the domain and range of his aggregation function that imply that the aggregation function only depends on one argument. For social welfare functions, this restriction corresponds to the existence of a dictator.
} 
tures of $x$. With the exception of some early work by Inada $(1964 ; 1971)$ and Pazner (1979), it is only recently that alternative independence conditions have been investigated for economic domains. See, for example, Fleurbaey and Maniquet (1996) and Fleurbaey, Suzumura, and Tadenuma (2002a).

Although lengthy, our discussion in Part I is not comprehensive. The results discussed in Part I have been extended in the following directions.

There is a large literature that examines the implications for Arrow's Theorem of weakening the assumption that social preferences are orderings and/or weakening (or dispensing with) the Pareto principle. On an unrestricted domain, the results obtained from this line of enquiry are rather negative. See Campbell and Kelly (2002). Similar negative results have been obtained for economic domains by, for example, Border (1983), Bordes and Le Breton (1989; 1990a; 1990b), Campbell (1989b; 1990a; 1990b; 1992b; 1992d; 1992e; 1996), and Nagahisa (1991). ${ }^{115}$

In Arrow's Theorem, it is assumed that there is a finite number of individuals. In many applications, such as overlapping generations economies, the society is infinite. With an unrestricted preference domain and an infinite society, social welfare functions exist that satisfy all of Arrow's axioms (although these functions have other unattractive features). Infinite societies with restricted preference domains have been considered by Campbell (1989a; 1989b; 1990b; 1992a; 1992b; 1992d) and Redekop (1995; 1996).

We have only considered alternatives that are either purely public or purely private. The mixed case in which alternatives have both public and private components has been considered by Bordes and Le Breton (1990b) and Redekop (1995; 1996).

A social welfare functional aggregates a profile of utility functions into a social preference. The use of a social welfare functional allows for information about interpersonal utility comparisons, when available, to play a role in determining the social ordering of the alternatives. Social welfare functionals on economic domains have been studied by Bordes, Hammond, and Le Breton (1997) and Weymark (1998).

The social choice correspondence framework permits us to consider restrictions on the set of feasible agendas, not just on the set of admissible preference profiles. Unlike with the local approach used to analyze social welfare functions, no single methodology has been identified that can be used to determine the consistency of Arrow's choice-theoretic axioms in a

\footnotetext{
${ }^{115}$ Many of these restricted domain theorems require social preferences to be continuous.
} 
wide variety of economic environments. However, a lesson that does emerge from the results we have surveyed in Part II is that the consistency of the Arrow axioms depends very much on how restrictive Independence of Infeasible Alternatives and Arrow's Choice Axiom are, and this depends on the structure of the agenda and preference domains. In some environments, one of these axioms is vacuous. For example, Independence of Infeasible Alternatives is vacuous for the economies with analytic preference domains and compact agenda domains considered in Section 15 and Arrow's Choice Axiom is vacuous in the exchange economies considered in Section 16.1. In such cases, it is very easy to construct social choice correspondences that satisfy all of the other Arrow axioms. Even when none of the axioms is vacuous, we have seen that possibility theorems emerge when the preference and/or agenda domains are sufficiently restricted.

Arrovian social choice theory on economic domains has a close affinity to the rapidly growing literature on the axiomatic analysis of resource allocation. ${ }^{116}$ As is the case with the literature discussed in Part II, the research on axiomatic models of resource allocation investigates the implications of a set of axioms when both individual preferences and the set of feasible agendas are assumed to satisfy the kinds of restrictions found in microeconomic models. However, it departs from the research discussed here in its choice of axioms and, in some cases, on the kinds of economic problems being considered. Many of the axioms advocated by practitioners of this approach to analyzing resource allocation problems are motivated by fairness considerations, incentive issues, or by the belief that the choices for different population sizes should be consistent with each other. For the most part, this literature has developed independently of the research we have surveyed and has typically not included all of the Arrow axioms in the set of axioms being investigated.

Of the four Arrow axioms for social choice correspondences, Arrow's Choice Axiom is the most controversial. We know from the work of Arrow (1959) and Hansson (1968) that, for some agenda domains, this axiom is equivalent to requiring the social choice correspondence to be rationalizable by a social welfare function. This is not true in general. When viewed as a constraint on the consistency of the choices made in different agendas, Arrow's Choice Axiom is a compelling normative property of a choice correspondence. It is interesting to note that, when combined with Strong Pareto, a version of the resource monotonicity axiom used in the literature

\footnotetext{
${ }^{116}$ See Moulin and Thomson (1997) for an introduction to this literature.
} 
on the axiomatic analysis of resource allocation implies a "welfarist" version of Arrow's Choice Axiom for social choice functions. Applied to abstract agenda domains, Resource Monotonicity requires that, for any fixed profile of preferences, nobody should be harmed by an expansion of the feasible set. ${ }^{117}$ For the social choice function $C$, consider a profile $\mathbf{R}$ and two agendas $A$ and $B$ with $A \subset B$ for which $C(B, \mathbf{R}) \in A$. Resource Monotonicity implies that $C(B, \mathbf{R}) R_{i} C(A, \mathbf{R})$ for all $i \in N$. From Strong Pareto and the assumption that $C(B, \mathbf{R}) \in A$, it then follows that $C(B, \mathbf{R}) I_{i} C(A, \mathbf{R})$ for all $i \in N$. Arrow's Choice Axiom is somewhat more demanding, requiring that $C(B, \mathbf{R})=C(A, \mathbf{R})$. This argument provides indirect support for requiring choices from different agendas to satisfy a choice consistency condition similar to the one used by Arrow. However, Resource Monotonicity is a demanding axiom that often conflicts with other appealing normative criteria. For example, for domains of exchange economies like those studied in Section 16.1, Moulin and Thomson (1988) have shown that any social choice function satisfying Resource Monotonicity and Weak Pareto must violate some very weak fairness axioms.

Our discussion of the theorems of Gibbard, Hylland, and Weymark (1987), Le Breton and Weymark (2002), and Donaldson and Weymark (1988) in Sections $13.3,15$, and 16.2 , respectively, demonstrates that when investigating the consistency of the Arrovian axioms on restricted domains, it matters a great deal whether one is dealing with a social welfare function or a social choice correspondence. Preference domains that result in an impossibility theorem using a social welfare function may well yield a possibility theorem using a social choice correspondence when combined with an appropriate agenda domain. ${ }^{118}$ It is standard practice in general equilibrium theory and in welfare economics to place a priori restrictions on both preferences and the set of feasible alternatives. For example, assumptions are often made on

\footnotetext{
${ }^{117}$ This property was introduced in the context of models in which the expansion of the feasible set was achieved by an increase in the economy's resource endowment.

${ }^{118}$ Marchant (2000) has suggested that this comparison is somewhat unfair because we are not comparing comparable objects. In particular, he argued that it would be more appropriate to make this comparison if the independence assumption for social welfare functions was restricted to apply only to a restricted set of agendas, as is the case with the independence assumption for social choice correspondences. Marchant's argument is consistent with our view that agenda domain restrictions can play an important role in the development of Arrovian possibility theorems. See also the discussion in Fleurbaey, Suzumura, and Tadenuma (2002b) of the relative strength of independence axioms in the social welfare function and social choice correspondence frameworks.
} 
production technologies and on endowments to ensure that the set of feasible alternatives is compact, comprehensive, and convex. The literature surveyed here suggests that pursuing a similar strategy in Arrovian social choice theory can be quite promising.

Much has been learned about the compatibility of Arrow's axioms in different economic environments. In our review of this literature, we have identified several open questions. Thus, in spite of the substantial progress that has be made, much more remains to be discovered about Arrovian social choice theory on economic domains.

\section{References}

Arrow, K. J., 1951. Social Choice and Individual Values. Wiley, New York.

Arrow, K. J., 1959. Rational choice functions and orderings. Economica 26, 121-127.

Arrow, K. J., 1963. Social Choice and Individual Values, 2nd Edition. Wiley, New York.

Austen-Smith, D., Banks, J. S., 1999. Positive Political Theory I: Collective Preference. University of Michigan Press, Ann Arbor.

Bailey, M. J., 1979. The possibility of rational social choice in an economy. Journal of Political Economy 87, 37-56.

Black, D., 1948. On the rationale of group decision-making. Journal of Political Economy 56, 23-34.

Blau, J. H., 1957. The existence of social welfare functions. Econometrica 25, 302-313.

Bone, J., 2003. Simple Arrow-type propositions in the Edgeworth domain. Social Choice and Welfare 20, 41-48.

Border, K. C., 1983. Social welfare functions for economic environments with and without the Pareto principle. Journal of Economic Theory 29, 205216.

Border, K. C., 1984. An impossibility theorem for spatial models. Public Choice 43, 293-305.

Border, K. C., Jordan, J. S., 1983. Straightforward elections, unanimity and phantom voters. Review of Economic Studies 50, 153-170.

Bordes, G., Campbell, D. E., Le Breton, M., 1995. Arrow's theorem for economic domains and Edgeworth hyperboxes. International Economic Review $36,441-454$. 
Bordes, G., Hammond, P. J., Le Breton, M., 1997. Social welfare functionals on restricted domains and in economic environments. Working Paper No. 97-023, Department of Economics, Stanford University.

Bordes, G., Le Breton, M., 1989. Arrovian theorems with private alternatives domains and selfish individuals. Journal of Economic Theory 47, 257-281.

Bordes, G., Le Breton, M., 1990a. Arrovian theorems for economic domains: Assignments, matchings and pairings. Social Choice and Welfare 7, 193208.

Bordes, G., Le Breton, M., 1990b. Arrovian theorems for economic domains: The case where there are simultaneously private and public goods. Social Choice and Welfare 7, 1-17.

Bossert, W., Weymark, J. A., 1993. Generalized median social welfare functions. Social Choice and Welfare 10, 17-33.

Campbell, D. E., 1989a. Arrow's theorem for economic environments and effective social preferences. Social Choice and Welfare 6, 325-329.

Campbell, D. E., 1989b. Wilson's theorem for economic environments and continuous social preferences. Social Choice and Welfare 7, 315-323.

Campbell, D. E., 1990a. Can equity be purchased at the expense of efficiency? An axiomatic inquiry. Journal of Economic Theory 51, 32-47.

Campbell, D. E., 1990b. Intergenerational social choice without the Pareto principle. Journal of Economic Theory 50, 414-423.

Campbell, D. E., 1992a. The Arrow and Plott independence conditions. Economics Letters 39, 143-146.

Campbell, D. E., 1992b. Equity, Efficiency, and Social Choice. Clarendon Press, Oxford.

Campbell, D. E., 1992c. Implementation of social welfare functions. International Economic Review 33, 525-533.

Campbell, D. E., 1992d. Quasitransitive intergenerational choice for economic environments. Journal of Mathematical Economics 21, 229-247.

Campbell, D. E., 1992e. Transitive social choice in economic environments. International Economic Review 33, 341-352.

Campbell, D. E., 1993. Euclidean individual preference and continuous social preference. European Journal of Political Economy 9, 541-550.

Campbell, D. E., 1995. Nonbinary social choice for economic environments. Social Choice and Welfare 12, 245-254.

Campbell, D. E., 1996. Social ranking of alternatives with and without coalition formation. In: Schofield, N. (Ed.), Collective Decision-Making: Social Choice and Political Economy. Kluwer Academic Publishers, Boston, pp. 
63-78.

Campbell, D. E., Kelly, J. S., 2000. Information and preference aggregation. Social Choice and Welfare 17, 3-24.

Campbell, D. E., Kelly, J. S., 2002. Impossibility theorems in the Arrovian framework. In: Arrow, K. J., Sen, A. K., Suzumura, K. (Eds.), Handbook of Social Choice and Welfare. Vol. 1. North-Holland, Amsterdam, pp. 3594.

Deb, R., 2004. Non-binary social choice. In: Arrow, K. J., Sen, A. K., Suzumura, K. (Eds.), Handbook of Social Choice and Welfare. Vol. 2. NorthHolland, Amsterdam.

Donaldson, D., Roemer, J. E., 1987. Social choice in economic environments with dimensional variation. Social Choice and Welfare 4, 253-276.

Donaldson, D., Weymark, J. A., 1988. Social choice in economic environments. Journal of Economic Theory 46, 291-308.

Duggan, J., 1996. Arrow's theorem in public goods environments with convex technologies. Journal of Economic Theory 68, 303-318.

Dutta, B., Jackson, M., Le Breton, M., 2001. Strategic candidacy and voting procedures. Econometrica 69, 1013-1037.

Ehlers, L., 2001. Independence axioms for the provision of multiple public goods as options. Mathematical Social Sciences 41, 239-250.

Ehlers, L., Storcken, T., 2002. Arrow's theorem in spatial environments. Cahier No. 2002-03, Département des sciences économiques, Université de Montréal.

Ehlers, L., Weymark, J. A., 2003. Candidate stability and nonbinary social choice. Economic Theory 22, 233-243.

Fishburn, P. C., 1976. Dictators on blocks: Generalization of social choice impossibility theorems. Journal of Combinatorial Theory, Series B 20, 153170.

Fishburn, P. C., Kelly, J. S., 1997. Super Arrovian domains with strict preferences. SIAM Journal on Discrete Mathematics 10, 83-95.

Fleurbaey, M., Maniquet, F., 1996. Utilitarianism versus fairness in welfare economics. In: Salles, M., Weymark, J. A. (Eds.), Justice, Political Liberalism, and Utilitarianism: Themes From Harsanyi and Rawls. Cambridge University Press, Cambridge, forthcoming.

Fleurbaey, M., Suzumura, K., Tadenuma, K., 2002a. Arrovian aggregation in economic environments: How much should we know about indifference surfaces? Discussion Paper No. 121, Project on Intergenerational Equity, Institute of Economic Research, Hitotsubashi University. 
Fleurbaey, M., Suzumura, K., Tadenuma, K., 2002b. The informational basis of the theory of fair allocation. Discussion Paper No. 122, Project on Intergenerational Equity, Institute of Economic Research, Hitotsubashi University.

Gaertner, W., 2001. Domain Conditions in Social Choice Theory. Cambridge University Press, Cambridge.

Gaertner, W., 2002. Domain restrictions. In: Arrow, K. J., Sen, A. K., Suzumura, K. (Eds.), Handbook of Social Choice and Welfare. Vol. 1. NorthHolland, Amsterdam, pp. 131-170.

Gibbard, A., Hylland, A., Weymark, J. A., 1987. Arrow's theorem with a fixed feasible alternative. Social Choice and Welfare 4, 105-115.

Grether, D. M., Plott, C. R., 1982. Nonbinary social choice: An impossibility theorem. Review of Economic Studies 49, 143-149.

Hansson, B., 1968. Choice structures and preference relations. Synthese 18, 443-458.

Inada, K., 1964. On the economic welfare function. Econometrica 32, 316338.

Inada, K., 1971. Social welfare function and social indifference surfaces. Econometrica 39, 599-623.

Kalai, E., Muller, E., Satterthwaite, M. A., 1979. Social welfare functions when preferences are convex, strictly monotonic, and continuous. Public Choice 34, 87-97.

Kalai, E., Ritz, Z., 1980. Characterization of the private alternatives domains admitting Arrow social welfare functions. Journal of Economic Theory 22, 23-36.

Kannai, Y., 1970. Continuity properties of the core of a market. Econometrica 38, 791-815.

Kannai, Y., 1974. Approximation of convex preferences. Journal of Mathematical Economics 1, 101-106.

Kelly, J. S., 1994. The Bordes-LeBreton exceptional case. Social Choice and Welfare 11, 273-281.

Lainé, J., Le Breton, M., Trannoy, A., 1986. Group decision making under uncertainty: A note on the aggregation of 'ordinal probabilities'. Theory and Decision 21, 155-161.

Le Breton, M., 1986. Essais sur les fondements de l'analyse économique de l'inégalité. Thèse pour le Doctorat d'État en Sciences Économiques, Université de Rennes 1.

Le Breton, M., 1997. Arrovian social choice on economic domains. In: Arrow, 
K. J., Sen, A. K., Suzumura, K. (Eds.), Social Choice Re-Examined. Vol. 1. Macmillan, London, pp. 72-96.

Le Breton, M., Trannoy, A., 1987. Measures of inequality as an aggregation of individual preferences about income distribution: The Arrovian case. Journal of Economic Theory 41, 248-269.

Le Breton, M., Weymark, J. A., 1996. An introduction to Arrovian social welfare functions on economic and political domains. In: Schofield, N. (Ed.), Collective Decision-Making: Social Choice and Political Economy. Kluwer Academic Publishers, Boston, pp. 25-61.

Le Breton, M., Weymark, J. A., 1999. Strategy-proof social choice functions with continuous separable preferences. Journal of Mathematical Economics $32,47-85$.

Le Breton, M., Weymark, J. A., 2002. Social choice with analytic preferences. Social Choice and Welfare 19, 637-657.

Marchant, T., 2000. Is there something more in SWF than in SCW?, unpublished manuscript, Department of Data Analysis, University of Ghent.

Maskin, E. S., 1976. Social welfare functions for economics, unpublished manuscript, Darwin College, Cambridge University and Department of Economics, Harvard University.

Mayston, D. J., 1980. Where did prescriptive welfare economics go wrong? In: Currie, D. A., Peters, W. (Eds.), Contemporary Economic Analysis. Vol. 2. Croom Helm, London, pp. 175-220.

Moulin, H., 1980. On strategy-proofness and single peakedness. Public Choice $35,437-455$.

Moulin, H., 1984. Generalized Condorcet-winners for single peaked and single-plateau preferences. Social Choice and Welfare 1, 127-147.

Moulin, H., Thomson, W., 1988. Can everyone benefit from growth? Two difficulties. Journal of Mathematical Economics 17, 339-345.

Moulin, H., Thomson, W., 1997. Axiomatic analysis of resource allocation problems. In: Arrow, K. J., Sen, A. K., Suzumura, K. (Eds.), Social Choice Re-Examined. Vol. 1. Macmillan, London, pp. 101-120.

Munkres, J. R., 1975. Topology: A First Course. Prentice-Hall, Englewood Cliffs, NJ.

Nagahisa, R., 1991. Acyclic and continuous social choice in $T_{1}$ connected spaces: Including its application to economic environments. Social Choice and Welfare 8, 319-322.

Nitzan, S., 1976. On linear and lexicographic orders: Majority rule and equilibrium. International Economic Review 17, 213-219. 
Pazner, E. A., 1979. Equity, nonfeasible alternatives and social choice: A reconsideration of the concept of social welfare. In: Laffont, J.-J. (Ed.), Aggregation and Revelation of Preferences. North-Holland, Amsterdam, pp. 161-173.

Peters, H., Wakker, P., 1991. Independence of irrelevant alternatives and revealed group preferences. Econometrica 59, 1787-1801.

Plott, C. R., 1976. Axiomatic social choice theory: An overview and interpretation. American Journal of Political Science 20, 511-596.

Redekop, J., 1991. Social welfare functions on restricted economic domains. Journal of Economic Theory 53, 396-427.

Redekop, J., 1993a. Arrow-inconsistent economic domains. Social Choice and Welfare 10, 107-126.

Redekop, J., 1993b. The questionnaire topology on some spaces of economic preferences. Journal of Mathematical Economics 22, 479-494.

Redekop, J., 1993c. Social welfare functions on parametric domains. Social Choice and Welfare 10, 127-148.

Redekop, J., 1995. Arrow theorems in economic environments. In: Barnett, W. A., Moulin, H., Salles, M., Schofield, N. J. (Eds.), Social Choice, Welfare, and Ethics. Cambridge University Press, Cambridge, pp. 163-185.

Redekop, J., 1996. Arrow theorems in mixed goods, stochastic, and dynamic economic environments. Social Choice and Welfare 13, 95-112.

Richelson, J. T., 1984. Social choice and the status quo. Public Choice 42, 225-234.

Saari, D. G., 1991. Calculus and extensions of Arrow's theorem. Journal of Mathematical Economics 20, 271-306.

Sen, A. K., 1970. Collective Choice and Social Welfare. Holden-Day, San Francisco.

Suzumura, K., 1983. Rational Choice, Collective Decisions, and Social Welfare. Cambridge University Press, Cambridge.

von Neumann, J., Morgenstern, O., 1947. Theory of Games and Economic Behavior, 2nd Edition. Princeton University Press, Princeton.

Weymark, J. A., 1997. Aggregating ordinal probabilities on finite sets. Journal of Economic Theory 75, 407-432.

Weymark, J. A., 1998. Welfarism on economic domains. Mathematical Social Sciences 36, 251-268.

Yanovskaya, E. B., 1994. Correspondence between social choice functions and solutions of cooperative games. Mathematical Social Sciences 27, 217-234. 


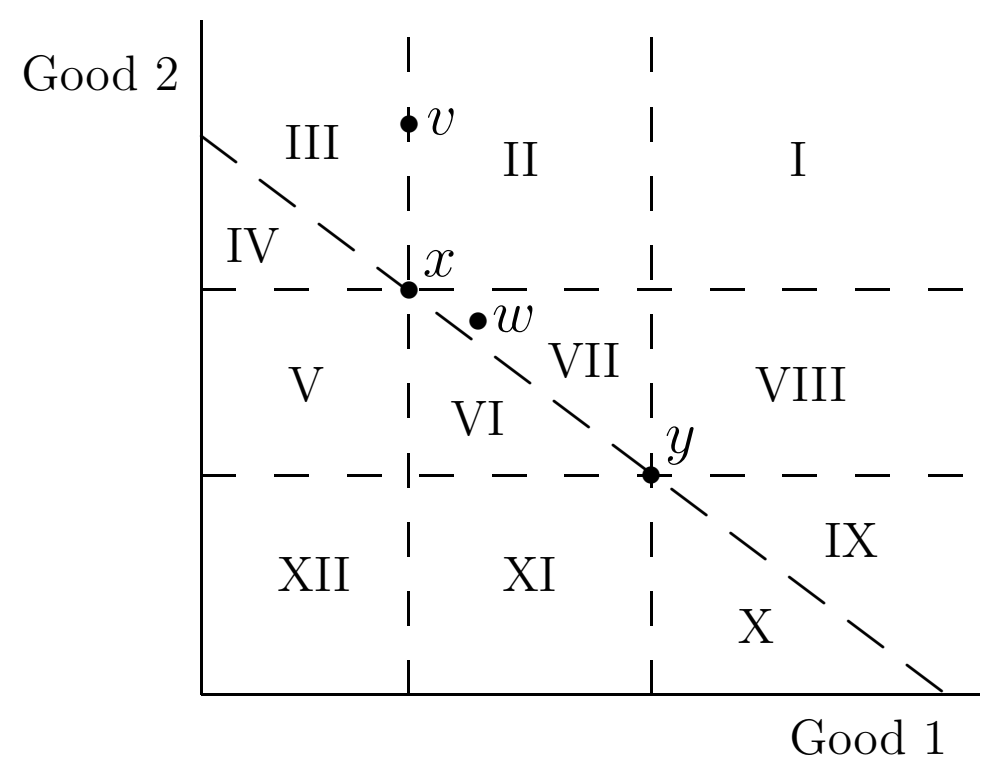

Figure 1

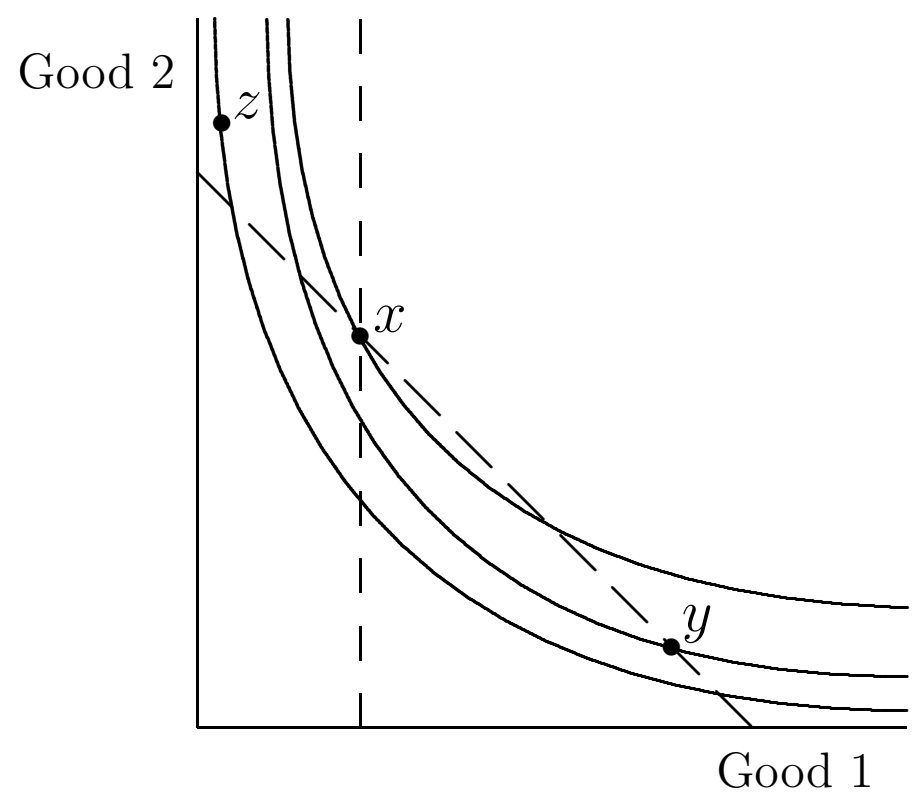

Figure 2 


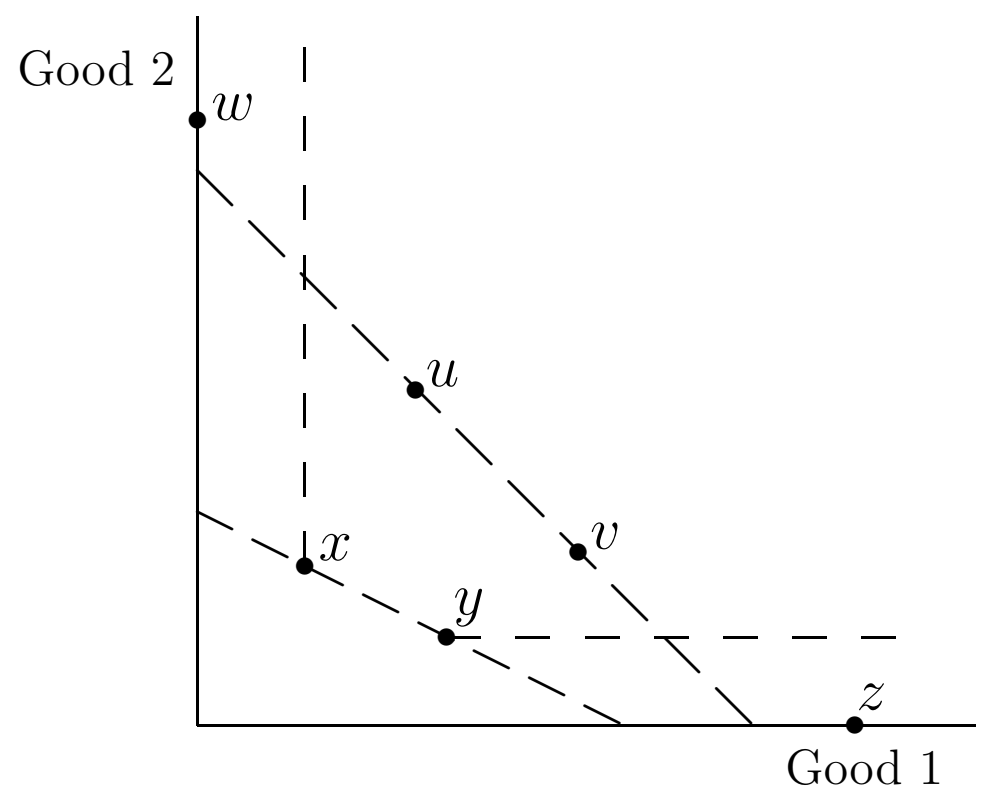

Figure 3

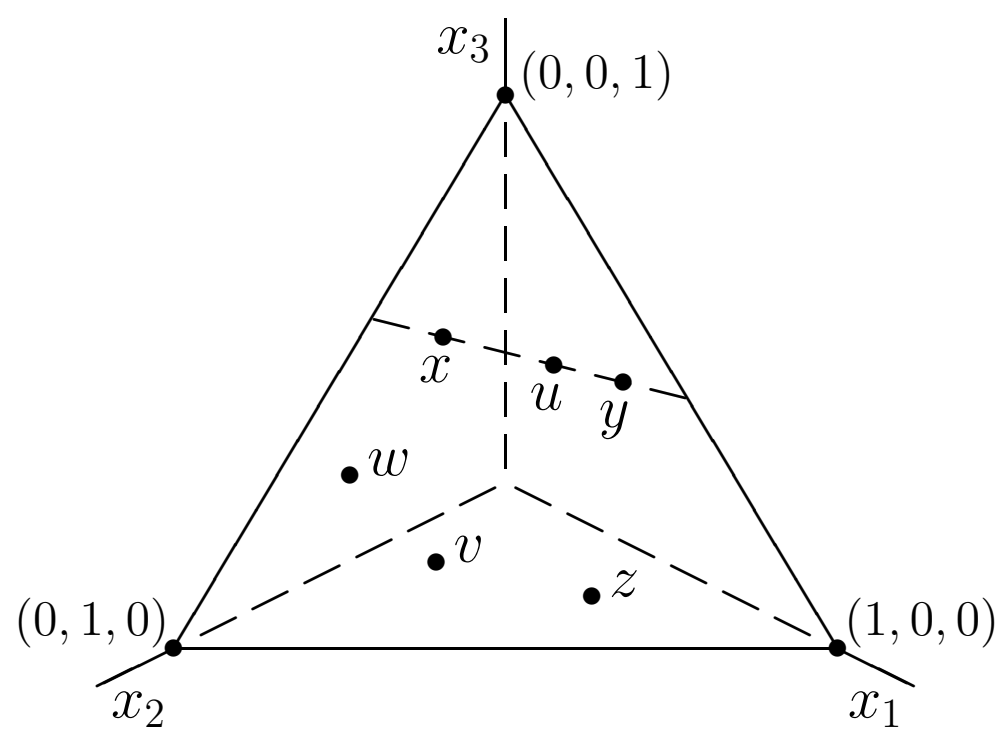

Figure 4 


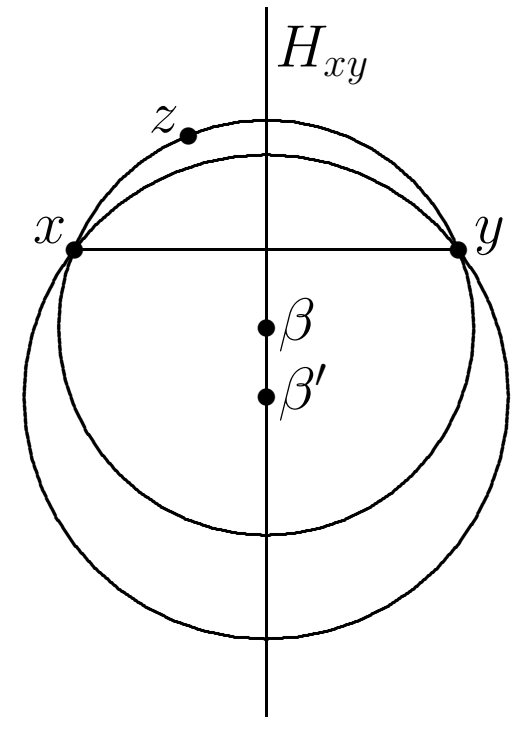

Figure 5

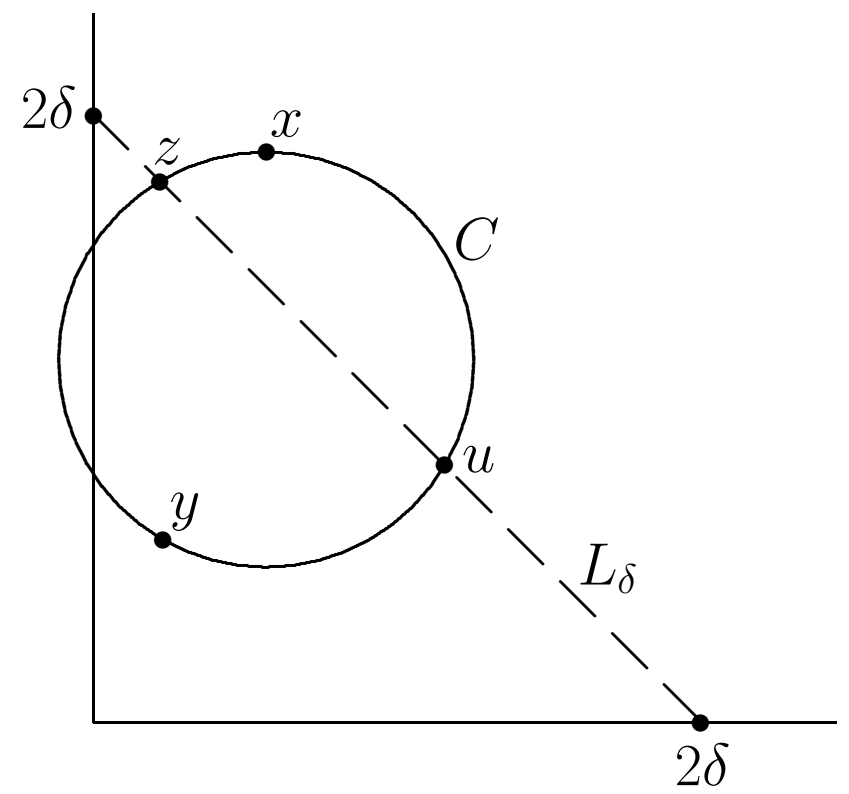

Figure 6 


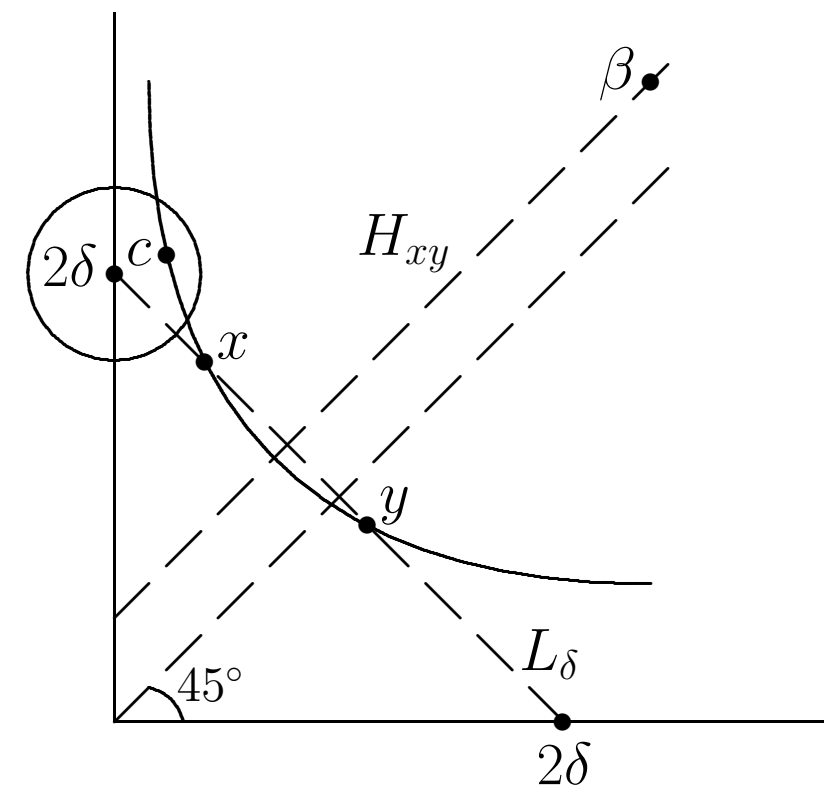

Figure 7

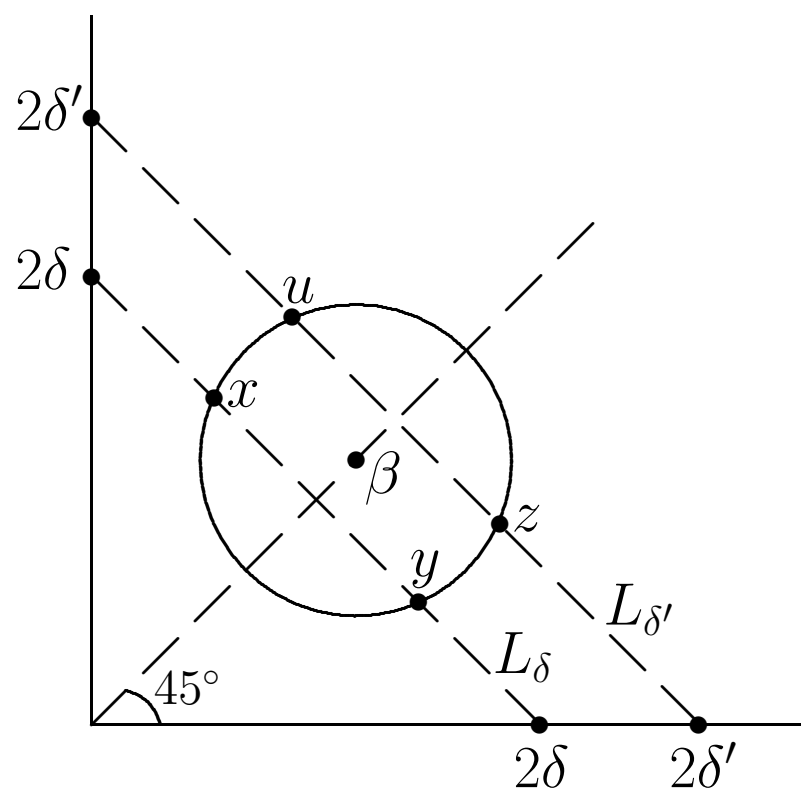

Figure 8 


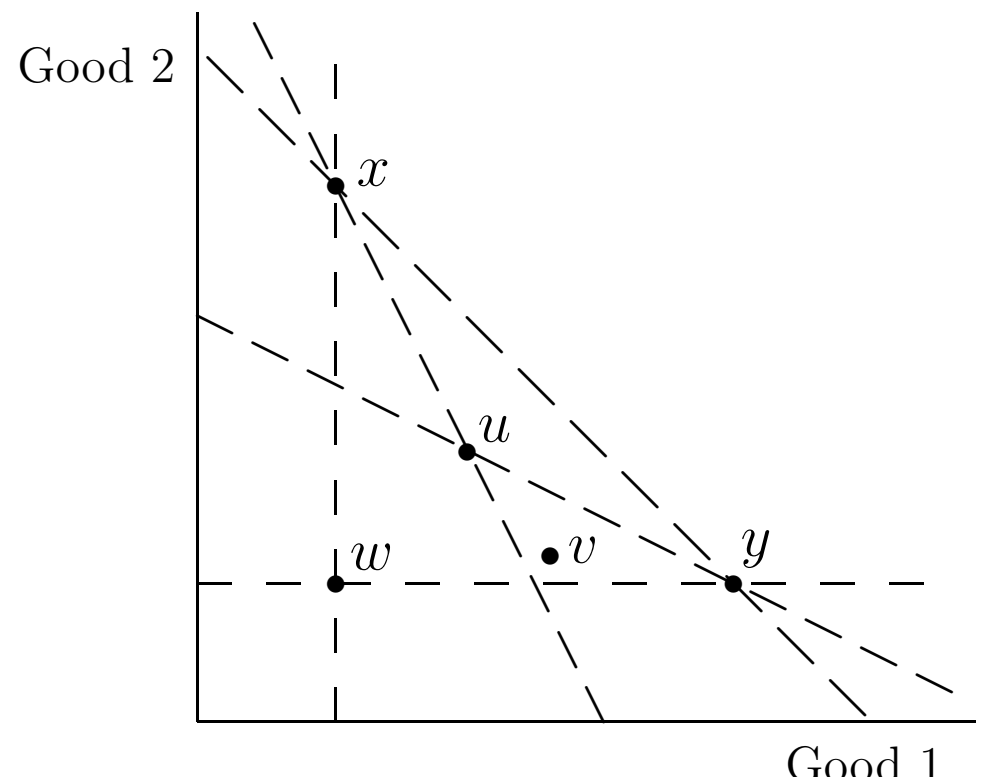

Figure 9

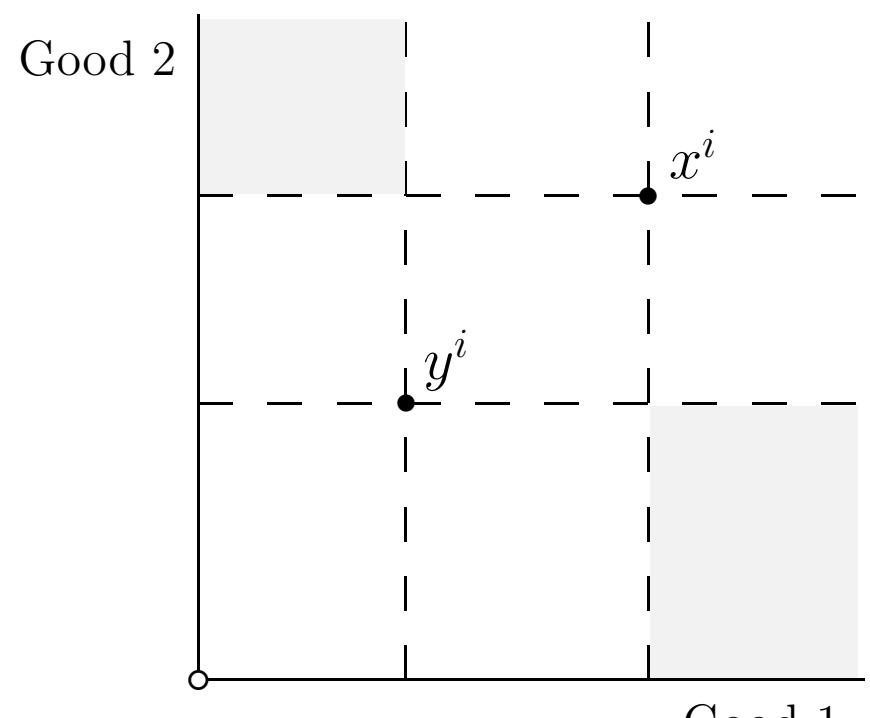

Figure 10 


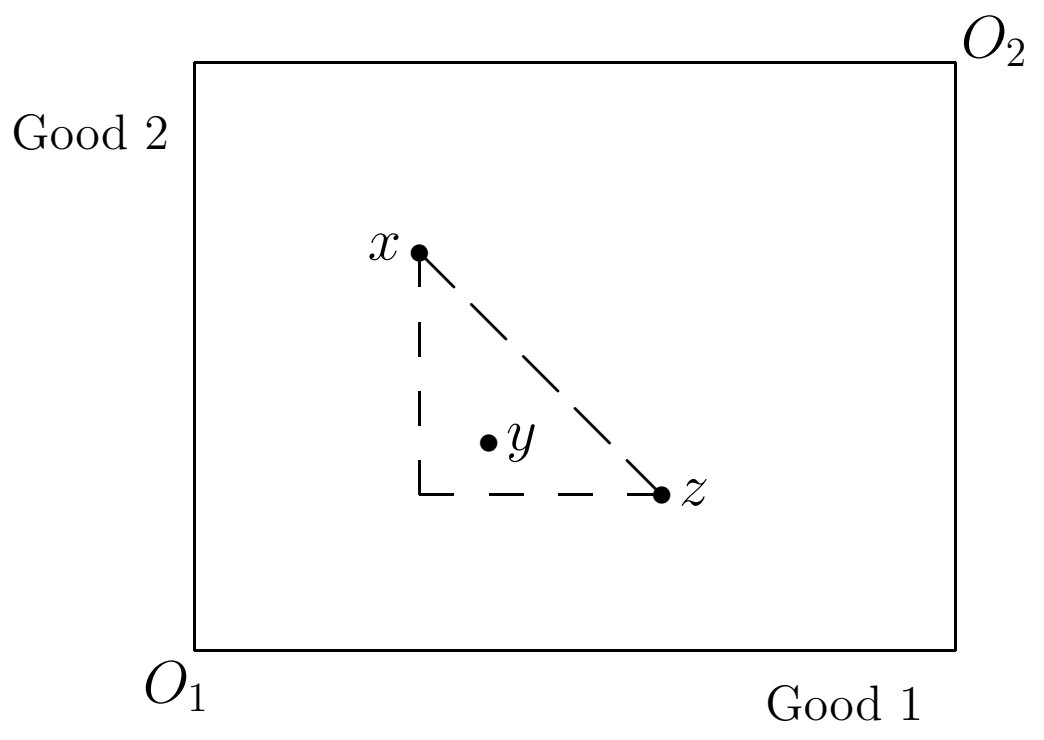

Figure 11

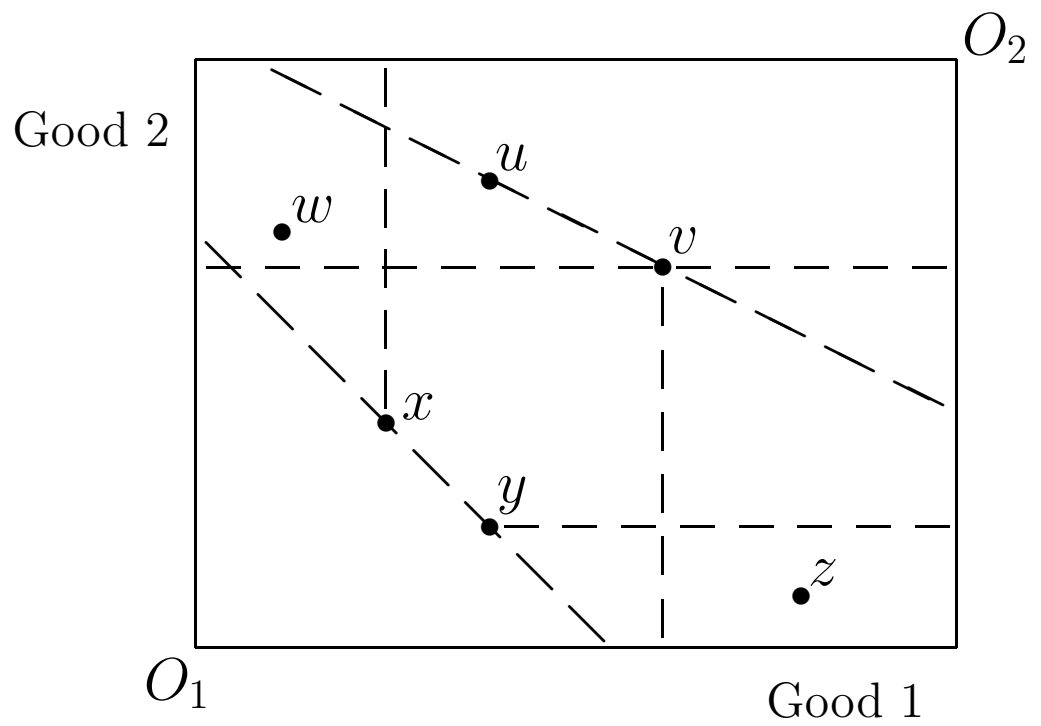

Figure 12 


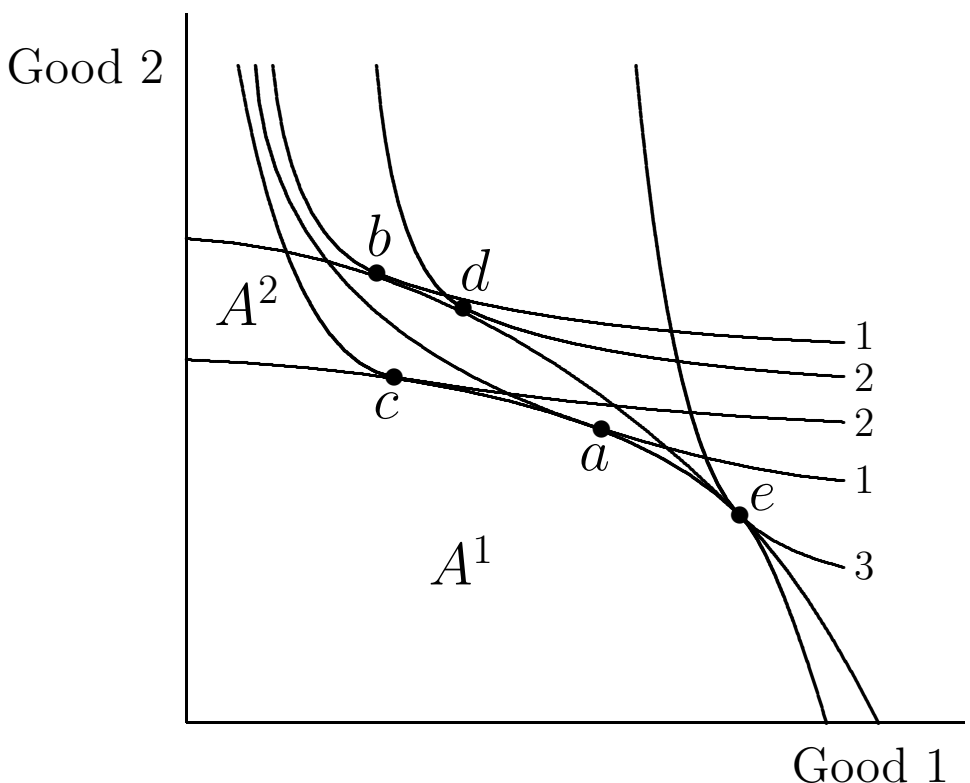

Figure 13 